Supporting Information:

\title{
C1-Galactopyranosyl heterocycle structure guides selectivity: Triazoles prefer galectin-1 and oxazoles prefer galectin-3
}

\author{
Alexander Dahlqvist ${ }^{1}$, Hakon Leffler ${ }^{2}$, Ulf J. Nilsson ${ }^{1 *}$ \\ ${ }^{1}$ Centre for Analysis and Synthesis, Department of Chemistry, Lund University. POB 124, 22100 , \\ Lund, Sweden \\ ${ }^{2}$ Section of Microbiology, Immunology and Glycobiology, Lund University. Sölvegatan 1922362 \\ Lund, Sweden \\ *Corresponding author: ulf.nilsson@chem.lu.se
}

Table of contents

Synthetic procedures and physical data for compounds $\mathbf{1 b}-\mathbf{1 j}, \mathbf{2 b}-\mathbf{2} \mathbf{i}, \mathbf{3 b}-\mathbf{3 c}, \mathbf{4 b}$ -

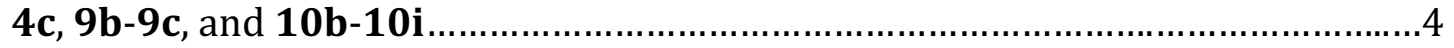

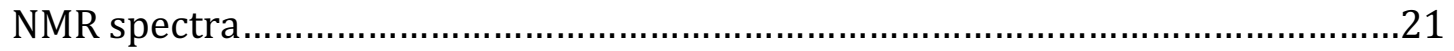


Synthetic procedures and physical data for compounds $\mathbf{1} \mathbf{b}-\mathbf{1} \mathbf{j}$, $2 b-2 i, 3 b-3 c, 4 b-4 c, 9 b-9 c$, and $10 b-10 i$

1-Naphth-1-yl-4-(1-deoxy- $\beta$-D-galactopyranosyl)-1H-1,2,3-triazole $1 \mathrm{~b}$<smiles>OC[C@H]1O[C@H](c2cn(-c3cccc4ccccc34)nn2)[C@H](O)[C@@H](O)[C@H]1O</smiles>

Compound 8 (100 mg, $0.280 \mathrm{mmol}$ ), copper(I) iodide (10 mg, $0.047 \mathrm{mmol}$ ) and 1-naphtyl azide (52 $\mathrm{mg}, 0.308 \mathrm{mmol}$ ) were dissolved in dry acetonitrile ( $3 \mathrm{~mL}$ ), triethylamine ( $51 \mu \mathrm{L}, 0.714 \mathrm{mmol})$ was added, and the reaction left overnight. The reaction was poured into ethyl acetate $(30 \mathrm{~mL})$ and washed with brine $(30 \mathrm{~mL})$. The brine was extracted twice with ethyl acetate $(30 \mathrm{~mL})$, the organic phases pooled, dried with anhydrous sodium sulfate and evaporated. The crude was dissolved in dry methanol ( $2 \mathrm{~mL}$ ) with sodium methoxide (85 mg, 1.57 mmol) and left for 3 hours. The reaction was quenched by Amberlite IR-120 ( $\left.\mathrm{H}^{+}\right)$ addition to $\mathrm{pH} 7$, filtered and evaporated. The crude was purified by column chromatography (5:1 dichloromethane/methanol) to give $\mathbf{1 b}(64 \mathrm{mg}, 66 \%)$. A prtion of $10 \mathrm{mg}$, 1b was purified with prep-HPLC (20 min gradient from 10\% acetonitrile $/ 90 \%$ water with $0.1 \%$ formic acid to $100 \%$ acetonitrile) prior to galectin binding evaluations.

${ }^{1} \mathrm{H}$ NMR (400 MHz, CD $\left.{ }_{3} \mathrm{OD}\right): \delta 8.42(\mathrm{~s}, 1 \mathrm{H}$, triazole $\mathrm{CH}), 8.18-8.12(\mathrm{~m}, 1 \mathrm{H}), 8.07(\mathrm{~d}$, $J=8.0 \mathrm{~Hz}, 1 \mathrm{H}), 7.71-7.56(\mathrm{~m}, 5 \mathrm{H}), 4.55\left(\mathrm{~d}, J=9.9 \mathrm{~Hz}, \mathbf{H}^{1}\right), 4.10-3.99(\mathrm{~m}, 2 \mathrm{H}), 3.87-$ $3.73(\mathrm{~m}, 3 \mathrm{H}) 3.69$ (dd, $J=9.7 \mathrm{~Hz}, 3.2 \mathrm{~Hz}, 1 \mathrm{H}, \mathbf{H}^{3}$ ) ${ }^{13} \mathrm{C}$ NMR (100 MHz, CD $\left.{ }_{3} \mathrm{OD}\right): \delta 167.24,146.56,134.27,133.52,130.36,128.44$, 128.05, 127.61, 126.87, 126.06, 124.85, 123.51, 121.80, 79.71, 74.97, 74.88, 70.90, 69.62, 61.55

HRMS: $\mathrm{M}+\mathrm{H}$; 358.1403 found, 358.1403 calculated. $[\alpha]^{20}{ }_{D}=16^{\circ}(c=0.70066$ in methanol $)$.

Purity by HPLC (UV/VIS detector $254 \mathrm{~nm}$ ): 99.6\%

1-naphth-2-yl-4-(1-deoxy- $\beta$-D-galactopyranosyl)-1H-1,2,3-triazole 1c<smiles>OC[C@H]1O[C@H](c2cn(-c3ccc4ccccc4c3)nn2)[C@H](O)[C@@H](O)[C@H]1O</smiles>

Compound 8 ( $48 \mathrm{mg}, 0.134 \mathrm{mmol}$ ), copper(I) iodide ( $5 \mathrm{mg}, 0.026 \mathrm{mmol}$ ) and 2naphtyl azide ( $25 \mathrm{mg}, 0.147 \mathrm{mmol}$ ) were dissolved in dry acetonitrile ( $3 \mathrm{~mL})$, triethylamine ( $25 \mu \mathrm{L}, 0.350 \mathrm{mmol})$ was added, and the reaction left overnight. The 
reaction was poured into ethyl acetate $(30 \mathrm{~mL})$ and washed with brine $(30 \mathrm{~mL})$. The brine was extracted twice with ethyl acetate $(30 \mathrm{~mL})$, the organic phases pooled, dried with anhydrous sodium sulfate and evaporated. The crude was dissolved in dry methanol ( $5 \mathrm{~mL})$ with sodium methoxide ( $85 \mathrm{mg}, 1.57 \mathrm{mmol})$ and left for 3 hours. The reaction was quenched by Amberlite IR-120 $\left(\mathrm{H}^{+}\right)$addition to $\mathrm{pH} 7$, filtered and evaporated. The crude was purified by column chromatography (5:1 dichloromethane/methanol) and prep-HPLC (20 min gradient from $10 \%$ acetonitrile/ $90 \%$ water with $0.1 \%$ formic acid to $100 \%$ acetonitrile) to give 1c (16 mg, 33\%).

${ }^{1} \mathrm{H}$ NMR (400 MHz, CD $\left.{ }_{3} \mathrm{OD}\right): \delta 8.72(\mathrm{~s}, 1 \mathrm{H}$, triazole $\mathrm{CH}), 8.36(\mathrm{~d}, J=1.8 \mathrm{~Hz}, 1 \mathrm{H}), 8.12$ $(\mathrm{d}, J=9.0 \mathrm{~Hz}, 1 \mathrm{H}), 8.05-7.98(\mathrm{~m}, 3 \mathrm{H}), 7.65-7.60(\mathrm{~m}, 2 \mathrm{H}), 4.50\left(\mathrm{~d}, J=9.9 \mathrm{~Hz}, \mathbf{H}^{1}\right)$, 4.06-3.98 (m, 2H), 3.83 (dd, $\left.J=12.3,8.7 \mathrm{~Hz}, 1 \mathrm{H}, \mathrm{H}^{6}\right) 3.78-3.73(\mathrm{~m}, 2 \mathrm{H}) 3.67$ (dd, $J=9.3 \mathrm{~Hz}, 3.4 \mathrm{~Hz}, 1 \mathrm{H}, \mathbf{H}^{3}$ )

${ }^{13} \mathrm{C}$ NMR (100 MHz, CD $\left.{ }_{3} \mathrm{OD}\right): \delta 129.76,128.02,127.61,127.18,126.81,121.69$, $118.43,118.11,79.68,75.00,74.86,70.89,69.58,61.52$

HRMS: M+H; 358.1412 found 358.1403 calculated.

$[\alpha]^{20}{ }_{D}=14^{\circ}(c=0.59177$ in methanol $)$.

Purity by HPLC (UV/VIS detector $254 \mathrm{~nm}$ ): 99.2\%

1-(4-Chlorophenyl)-4-(1-deoxy- $\beta$-D-galactopyranosyl)-1H-1,2,3-triazole $1 \mathrm{~d}$

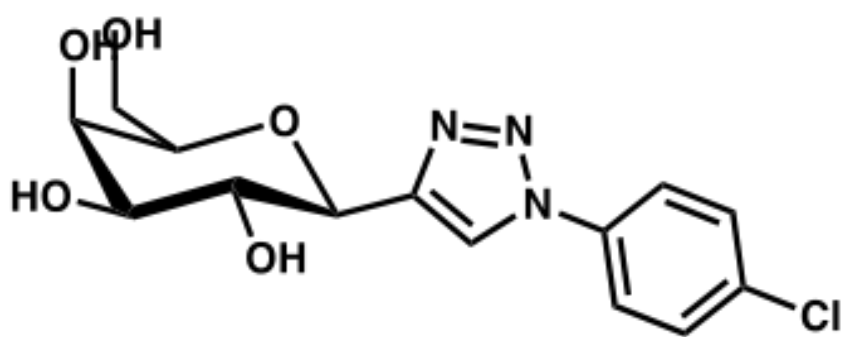

Compound 8 (25 mg, $0.070 \mathrm{mmol}$ ) was dissolved in dry acetonitrile ( $2 \mathrm{~mL}$ ) with copper(I) iodide ( $3 \mathrm{mg}, 0.014 \mathrm{mmol}$ ). 4-Chlorophenyl azide (0.15 mL, 0.074 mmol, $0.5 \mathrm{M}$ in tert-butyl methyl ether) and triethylamine (10 $\mu \mathrm{L}, 0.140 \mathrm{mmol})$ were added. The reaction was left overnight at room temperature, then poured into ethyl acetate $(20 \mathrm{~mL})$ and washed with brine $(20 \mathrm{~mL})$. The brine was extracted twice with ethyl acetate $(20 \mathrm{~mL})$, the organic phases pooled, dried with anhydrous sodium sulfate and evaporated. The crude was dissolved inof dry methanol ( $2 \mathrm{~mL})$ with sodium methoxide (23 $\mathrm{mg}, 0.420 \mathrm{mmol})$ and left for 3 hours. The reaction was quenched with Amberlite IR-120 $\left(\mathrm{H}^{+}\right)$until pH 7, filtered and evaporated. The crude was purified with prep-HPLC (20 min gradient from $10 \%$ acetonitrile/ $90 \%$ water with $0.1 \%$ formic acid to $100 \%$ acetonitrile) to give 1d (7 mg, 30\%).

${ }^{1} \mathrm{H}$ NMR (400 MHz, $\left.\mathrm{CD}_{3} \mathrm{OD}\right): \delta 8.58(\mathrm{~s}, 1 \mathrm{H}$, triazole $\mathrm{CH}$ ), 7.91-7.86 $(\mathrm{m}, 2 \mathrm{H}), 7.65-$ $7.60(\mathrm{~m}, 2 \mathrm{H}), 4.45\left(\mathrm{~d}, J=9.7 \mathrm{~Hz}, \mathrm{H}^{1}\right), 4.02-3.95(\mathrm{~m}, 2 \mathrm{H}), 3.81(\mathrm{dd}, \mathrm{J} 1=11.7 \mathrm{~Hz}$, $\left.\mathrm{J} 2=8.2,1 \mathrm{H}, \mathbf{H}^{6}\right), 3.76-3.71(\mathrm{~m}, 2 \mathrm{H}), 3.65\left(\mathrm{dd}, \mathrm{J} 1=9.6 \mathrm{~Hz}, \mathrm{~J} 2=2.9 \mathrm{~Hz}, 1 \mathrm{H}, \mathbf{H}^{3}\right.$ ) ${ }^{13} \mathrm{C}$ NMR (100 MHz, CD ${ }_{3}$ OD): $\delta 135.73,134.26,129.66,121.62,121.54,79.66$ (C5), 74.92 (C1), 74.82 (C3), 70.85 (C2), 69.54 (C4), 61.49 (C6)

HRMS: M+H; 342.0857 found, 342.0857 calculated. $[\alpha]^{20}{ }_{D}=25^{\circ}(c=0.22288$ in methanol $)$.

Purity by HPLC (UV/VIS detector $254 \mathrm{~nm}$ ): 99.8\% 
<smiles>OC[C@H]1O[C@H](c2cn(-c3cccc(Cl)c3)nn2)[C@H](O)[C@@H](O)[C@H]1O</smiles>

Compound 8 (25 mg, $0.070 \mathrm{mmol}$ ) was dissolved in dry acetonitrile ( $2 \mathrm{~mL}$ ) with copper(I) iodide (3 mg, $0.014 \mathrm{mmol})$. 3-Chlorophenyl azide $(0.15 \mathrm{~mL}, 0.074$ mmol, $0.5 \mathrm{M}$ in tert-butyl methyl ether $)$ and triethylamine $(10 \mu \mathrm{L}, 0.140 \mathrm{mmol})$ were added. The reaction was left overnight at room temperature, then poured into ethyl acetate $(20 \mathrm{~mL})$ and washed with brine $(20 \mathrm{~mL})$. The brine was extracted twice with ethyl acetate $(20 \mathrm{~mL})$, the organic phases pooled, dried with anhydrous sodium sulfate and evaporated. The crude was dissolved inof dry methanol ( $2 \mathrm{~mL}$ ) with sodium methoxide (23 $\mathrm{mg}, 0.420 \mathrm{mmol})$ and left for 3 hours. The reaction was quenched with Amberlite IR-120 $\left(\mathrm{H}^{+}\right)$until pH 7, filtered and evaporated. The crude was purified with prep-HPLC (20 min gradient from $10 \%$ acetonitrile $/ 90 \%$ water with $0.1 \%$ formic acid to $100 \%$ acetonitrile) to give 1e (24 mg, 99\%).

${ }^{1} \mathrm{H}$ NMR (400 MHz, CD $\left.{ }_{3} \mathrm{OD}\right): \delta 8.63(\mathrm{~s}, 1 \mathrm{H}$, triazole $\mathrm{CH}), 7.97(\mathrm{t}, J=2.0 \mathrm{~Hz}, 1 \mathrm{H}), 7.83$ $(\mathrm{dq}, J=4.1 \mathrm{~Hz}, 1.0 \mathrm{~Hz}, 1 \mathrm{H}), 7.57(\mathrm{t}, J=8 \mathrm{~Hz}, 1 \mathrm{H}), 7.53(\mathrm{dq}, \mathrm{J1}=4 \mathrm{~Hz}, 1 \mathrm{~Hz}, 1 \mathrm{H}), 4.46$ (d, $J=9.7 \mathrm{~Hz}, \mathbf{H}^{1}$ ), 4.03-3.95 (m, 2H), $3.81\left(\mathrm{dd}, J=11.7 \mathrm{~Hz}, 8.2 \mathrm{~Hz}, 1 \mathrm{H}, \mathbf{H}^{6}\right), 3.77-3.71$ (m, 2H), $3.66\left(\mathrm{dd}, J=9.07 \mathrm{~Hz}, 2.9 \mathrm{~Hz}, 1 \mathrm{H}, \mathbf{H}^{3}\right)$ ${ }^{13} \mathrm{C}$ NMR (100 MHz, CD $\left.{ }_{3} \mathrm{OD}\right): \delta 135.73,134.26,129.66,121.62,121.54,79.66$, 74.92, 74.82, 70.85, 69.54, 61.49

HRMS: $\mathrm{M}+\mathrm{H}$; 342.0857 found, 342.0857 calculated. $[\alpha]^{20}{ }_{D}=15^{\circ}(c=0.55322$ in methanol $)$.

Purity by HPLC (UV/VIS detector $254 \mathrm{~nm}$ ): 95.1\%

1-(4-Methylphenyl)-4-(1-deoxy- $\beta$-D-galactopyranosyl)-1H-1,2,3-triazole $1 \mathrm{f}$

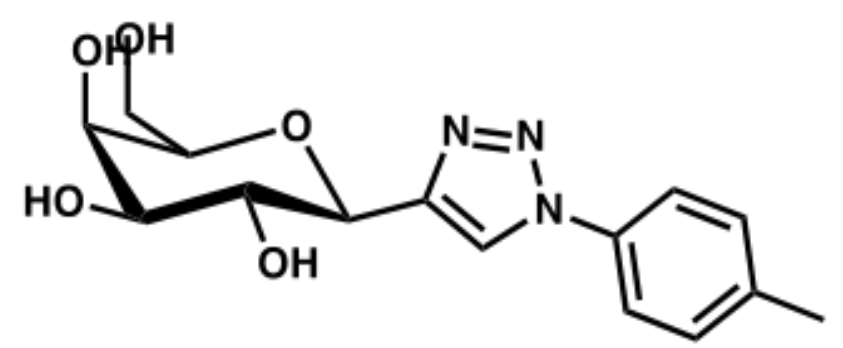

Compound 8 (25 mg, $0.070 \mathrm{mmol}$ ) was dissolved in dry acetonitrile ( $2 \mathrm{~mL}$ ) with copper(I) iodide (3 mg, $0.014 \mathrm{mmol})$. 4-Methylphenyl azide $(0.15 \mathrm{~mL}, 0.074$ mmol, $0.5 \mathrm{M}$ in tert-butyl methyl ether) and triethylamine (10 $\mu \mathrm{L}, 0.140 \mathrm{mmol})$ were added. The reaction was left overnight at room temperature, then poured into ethyl acetate $(20 \mathrm{~mL})$ and washed with brine $(20 \mathrm{~mL})$. The brine was extracted twice with ethyl acetate $(20 \mathrm{~mL})$, the organic phases pooled, dried with anhydrous sodium sulfate and evaporated. The crude was dissolved inof dry methanol ( $2 \mathrm{~mL}$ ) with sodium methoxide (23 mg, $0.420 \mathrm{mmol})$ and left for 3 hours. The reaction was quenched with Amberlite IR-120 $\left(\mathrm{H}^{+}\right)$until pH 7, filtered 
and evaporated. The crude was purified with prep-HPLC (20 min gradient from $10 \%$ acetonitrile $/ 90 \%$ water with $0.1 \%$ formic acid to $100 \%$ acetonitrile) to give 1f (4 mg, 16\%).

${ }^{1} \mathrm{H}$ NMR $\left(400 \mathrm{MHz}, \mathrm{CD}_{3} \mathrm{OD}\right): \delta 8.51(\mathrm{~s}, 1 \mathrm{H}$, triazole $\mathrm{CH}), 7.75-7.70(\mathrm{~m}, 2 \mathrm{H}), 7.44-$ $7.39(\mathrm{~m}, 2 \mathrm{H}), 4.45\left(\mathrm{~d}, J=9.9 \mathrm{~Hz}, \mathbf{H}^{1}\right), 4.02-3.95(\mathrm{~m}, 2 \mathrm{H}), 3.81$ (dd, $J=11.7 \mathrm{~Hz}, 8.2 \mathrm{~Hz}$, $\left.1 \mathrm{H}, \mathbf{H}^{6}\right), 3.76-3.71(\mathrm{~m}, 2 \mathrm{H}), 3.65\left(\mathrm{dd}, J=9.1 \mathrm{~Hz}, 2.9 \mathrm{~Hz}, 1 \mathrm{H}, \mathbf{H}^{3}\right), 2.45$ (s, 3H, phenyl methyl $\mathrm{H}_{3}$ )

${ }^{13} \mathrm{C}$ NMR $\left(100 \mathrm{MHz}, \mathrm{CD}_{3} \mathrm{OD}\right): \delta 147.05,139.14,134.74,129.99,121.51,120.12$,

79.63, 74.94, 74.83, 70.86, 69.56, 61.49, 19.67

HRMS: $\mathrm{M}+\mathrm{H} ; 322.1399$ found, 322.1403 calculated.

$[\alpha]^{20}{ }_{D}=16^{\circ}(c=0.12387$ in methanol $)$.

Purity by HPLC (UV/VIS detector $254 \mathrm{~nm}$ ): 96.6\%

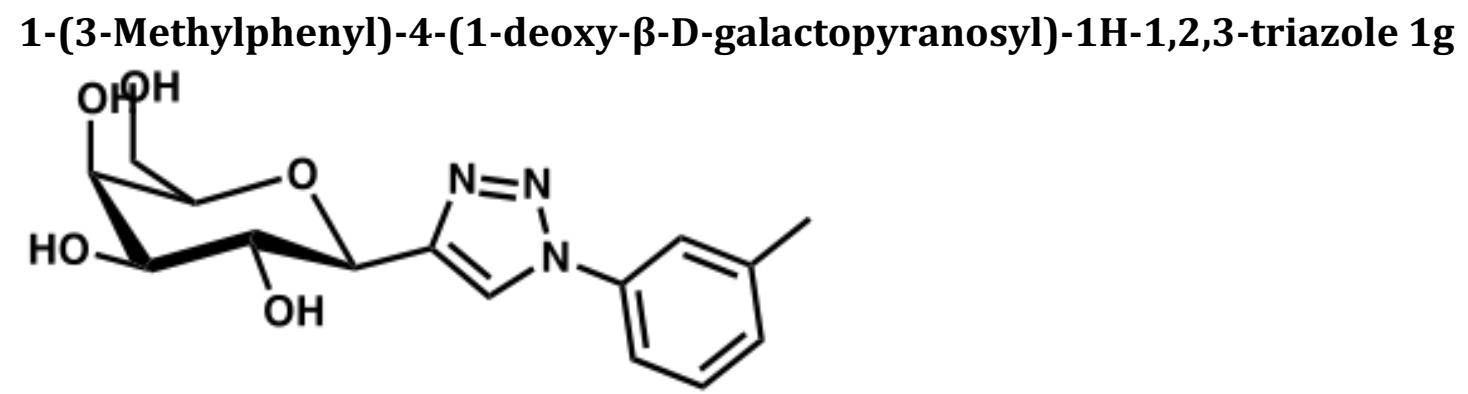

Compound 8 (25 mg, $0.070 \mathrm{mmol})$ was dissolved in dry acetonitrile $(2 \mathrm{~mL})$ with copper(I) iodide (3 mg, $0.014 \mathrm{mmol})$. 3-Methylphenyl azide $(0.15 \mathrm{~mL}, 0.074$ mmol, $0.5 \mathrm{M}$ in tert-butyl methyl ether) and triethylamine $(10 \mu \mathrm{L}, 0.140 \mathrm{mmol})$ were added. The reaction was left overnight at room temperature, then poured into ethyl acetate $(20 \mathrm{~mL})$ and washed with brine $(20 \mathrm{~mL})$. The brine was extracted twice with ethyl acetate $(20 \mathrm{~mL})$, the organic phases pooled, dried with anhydrous sodium sulfate and evaporated. The crude was dissolved inof dry methanol ( $2 \mathrm{~mL}$ ) with sodium methoxide ( $23 \mathrm{mg}, 0.420 \mathrm{mmol})$ and left for 3 hours. The reaction was quenched with Amberlite IR-120 $\left(\mathrm{H}^{+}\right)$until pH 7, filtered and evaporated. The crude was purified with prep-HPLC $(20$ min gradient from $10 \%$ acetonitrile $/ 90 \%$ water with $0.1 \%$ formic acid to $100 \%$ acetonitrile) to give $1 \mathrm{~g}$ (4 mg, 19\%).

${ }^{1} \mathrm{H}$ NMR $\left(400 \mathrm{MHz}, \mathrm{CD}_{3} \mathrm{OD}\right): \delta 8.54(\mathrm{~s}, 1 \mathrm{H}$, triazole $\mathrm{CH}), 7.72-7.69(\mathrm{~m}, 1 \mathrm{H}), 7.67-$ $7.63(\mathrm{~m}, 1 \mathrm{H}), 7.48(\mathrm{t}, J=7.8 \mathrm{~Hz}, 1 \mathrm{H}), 7.37-7.33(\mathrm{~m}, 1 \mathrm{H}), 4.46\left(\mathrm{~d}, J=9.7 \mathrm{~Hz}, \mathbf{H}^{1}\right), 4.02-$ $3.95(\mathrm{~m}, 2 \mathrm{H}), 3.81\left(\mathrm{dd}, J=12.2 \mathrm{~Hz}, 8.2 \mathrm{~Hz}, 1 \mathrm{H}, \mathbf{H}^{6}\right), 3.76-3.71(\mathrm{~m}, 2 \mathrm{H}), 3.65$ (dd, $J=9.1 \mathrm{~Hz}, 3.1 \mathrm{~Hz}, 1 \mathrm{H}, \mathbf{H}^{3}$ ), $2.48\left(\mathrm{~s}, 3 \mathrm{H}\right.$, phenyl methyl $\mathrm{H}_{3}$ )

${ }^{13} \mathrm{C}$ NMR $\left(100 \mathrm{MHz}, \mathrm{CD}_{3} \mathrm{OD}\right): \delta 137.19,129.39,121.55,120.67,117.28,79.65$, 74.94, 74.83, 70.85, 69.56, 61.49, 19.98

HRMS: $\mathrm{M}+\mathrm{H} ; 322.1402$ found, 322.1403 calculated. $[\alpha]^{20}{ }_{D}=13^{\circ}(c=0.16830$ in methanol $)$.

Purity by HPLC (UV/VIS detector $254 \mathrm{~nm}$ ): 99.6\% 


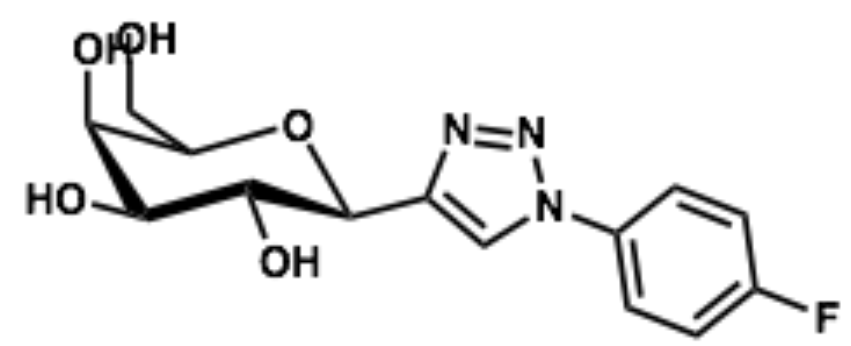

Compound 8 (25 mg, $0.070 \mathrm{mmol}$ ), was dissolved in dry acetonitrile ( $2 \mathrm{~mL}$ ) with copper(I) iodide ( $3 \mathrm{mg}, 0.014 \mathrm{mmol})$. 4-Fluorophenyl azide $(0.15 \mathrm{~mL}, 0.074$ mmol, $0.5 \mathrm{M}$ in tert-butyl methyl ether) and triethylamine (10 $\mu \mathrm{L} 0.140 \mathrm{mmol})$ were added. The reaction was left overnight at room temperature, then poured into ethyl acetate $(20 \mathrm{~mL})$ and washed with brine $(20 \mathrm{~mL})$. The brine was extracted twice with ethyl acetate $(20 \mathrm{~mL})$, the organic phases pooled, dried with anhydrous sodium sulfate and evaporated. The crude was dissolved inof dry methanol ( $2 \mathrm{~mL}$ ) with sodium methoxide (23 $\mathrm{mg}, 0.420 \mathrm{mmol})$ and left for 3 hours. The reaction was quenched with Amberlite IR-120 $\left(\mathrm{H}^{+}\right)$until pH 7, filtered and evaporated. The crude was purified with prep-HPLC (20 min gradient from $10 \%$ acetonitrile/ $90 \%$ water with $0.1 \%$ formic acid to $100 \%$ acetonitrile) to give 1h (11 mg, 44\%).

${ }^{1} \mathrm{H}$ NMR (400 MHz, CD $\left.{ }_{3} \mathrm{OD}\right): \delta 8.54(\mathrm{~s}, 1 \mathrm{H}$, triazole $\mathrm{CH}$ ), 7.89-7.86 (m, 2H), 7.40$7.32(\mathrm{~m}, 2 \mathrm{H}), 4.46$ (d, J=9.7 Hz, H1 $), 4.01-3.95(\mathrm{~m}, 2 \mathrm{H}), 3.81$ (dd, J=11.7 Hz, $8.2 \mathrm{~Hz}$, $1 \mathrm{H}, \mathbf{H}^{6}$ ), 3.76-3.71 (m, 2H), 3.65 (dd, J=9.7 Hz, $2.9 \mathrm{~Hz}, 1 \mathrm{H}, \mathbf{H}^{3}$ )

${ }^{13} \mathrm{C}$ NMR (100 MHz, CD ${ }_{3}$ OD): $\delta 163.81,161.35,147.28,133.45,122.49,122.41$, 121.76, 116.43, 116.19, 79.65, 74.93, 74.82, 70.85, 69.55, 61.57

HRMS: M+Na; 348.0972 found, 348.0972 calculated.

$[\alpha]^{20}{ }_{D}=22^{\circ}(c=0.33000$ in methanol $)$.

Purity by HPLC (UV/VIS detector $254 \mathrm{~nm}$ ): 99.9\%

1-(3-Fluorophenyl)-4-(1-deoxy- $\beta$-D-galactopyranosyl)-1H-1,2,3-triazole 1i<smiles>OC[C@H]1O[C@H](c2cn(-c3cccc(F)c3)nn2)[C@H](O)[C@@H](O)[C@H]1O</smiles>

Compound 8 (25 mg, $0.070 \mathrm{mmol}$ ) was dissolved in dry acetonitrile ( $2 \mathrm{~mL}$ ) with copper(I) iodide (3 mg, $0.014 \mathrm{mmol}$ ). 3-Fluorophenyl azide $(0.15 \mathrm{~mL}, 0.074$ mmol, 0.5 $\mathrm{M}$ in tert-butyl methyl ether $)$ and triethylamine $(10 \mu \mathrm{L}, 0.140 \mathrm{mmol})$ were added. The reaction was left overnight at room temperature, then poured into ethyl acetate $(20 \mathrm{~mL})$ and washed with brine $(20 \mathrm{~mL})$. The brine was extracted twice with ethyl acetate $(20 \mathrm{~mL})$, the organic phases pooled, dried with anhydrous sodium sulfate and evaporated. The crude was dissolved inof dry methanol ( $2 \mathrm{~mL}$ ) with sodium methoxide (23 $\mathrm{mg}, 0.420 \mathrm{mmol})$ and left for 3 
hours. The reaction was quenched with Amberlite IR-120 $\left(\mathrm{H}^{+}\right)$until pH 7, filtered and evaporated. The crude was purified with prep-HPLC (20 min gradient from $10 \%$ acetonitrile $/ 90 \%$ water with $0.1 \%$ formic acid to $100 \%$ acetonitrile) to give $1 \mathrm{i}(14.7 \mathrm{mg}, 64 \%$ ) as a lemon-yellow solid.

${ }^{1} \mathrm{H}$ NMR (400 MHz, CD $\left.{ }_{3} \mathrm{OD}\right): \delta 8.62(\mathrm{~s}, 1 \mathrm{H}$, triazole $\mathrm{CH}), 7.76-7.70(\mathrm{~m}, 2 \mathrm{H}), 7.67-$ 7.59 (m, 1H), (tdd, J= 8.4 Hz, 2.5 Hz, 0.9 Hz, 1H), 4.46 (d, J=10.0 Hz, H' ${ }^{1}$ ), 4.02-3.94 (m, 2H), $3.81\left(\mathrm{dd}, J=12.1 \mathrm{~Hz}, 8.4 \mathrm{~Hz}, \mathbf{H}^{6}\right), 3.77-3.70(\mathrm{~m}, 2 \mathrm{H}), 3.64(\mathrm{dd}, J=9.5 \mathrm{~Hz}, 3.3$ $\left.\mathrm{Hz}, \mathbf{H}^{3}\right)$

${ }^{13} \mathrm{C}$ NMR (100 MHz, CD $\left.{ }_{3} \mathrm{OD}\right): \delta 131.42,131.33,121.62,115.71,115.38,115.16$, $107.7,107.44,79.67,74.91,74.82,70.84,69.55,61.49$

HRMS: $\mathrm{M}+\mathrm{H}$; 326.1155 found, 326.1152 calculated. $[\alpha]^{20}{ }_{D}=24^{\circ}(c=0.35414$ in methanol. $)$

Purity by HPLC (UV/VIS detector $254 \mathrm{~nm}$ ): 99.7\%

\section{1-(2-Fluorophenyl)-4-(1-deoxy- $\beta$-D-galactopyranosyl)-1H-1,2,3-triazole 1j}

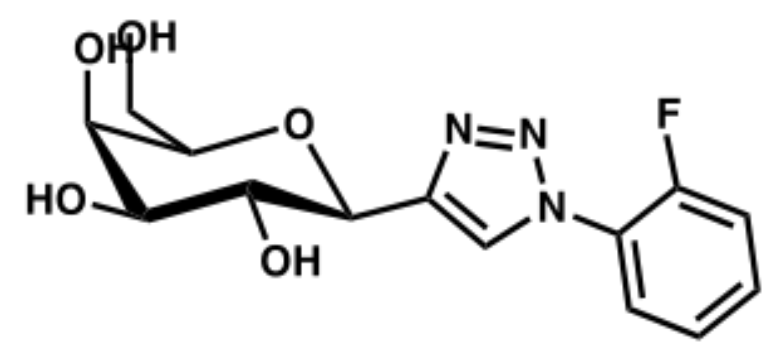

Compound 8 (25 mg, $0.070 \mathrm{mmol}$ ) was dissolved in dry acetonitrile ( $2 \mathrm{~mL}$ ) with copper(I) iodide (3 mg, $0.014 \mathrm{mmol})$. Phenyl azide $(0.15 \mathrm{~mL}, 0.074 \mathrm{mmol}, 0.5 \mathrm{M}$ in tert-butyl methyl ether) and triethylamine (10 $\mu \mathrm{L}, 0.140 \mathrm{mmol})$ were added. The reaction was left overnight at room temperature, then poured into ethyl acetate $(20 \mathrm{~mL})$ and washed with brine $(20 \mathrm{~mL})$. The brine was extracted twice with ethyl acetate $(20 \mathrm{~mL})$, the organic phases pooled, dried with anhydrous sodium sulfate and evaporated. The crude was dissolved inof dry methanol ( $2 \mathrm{~mL})$ with sodium methoxide ( $23 \mathrm{mg}, 0.420 \mathrm{mmol}$ ) and left for 3 hours. The reaction was quenched with Amberlite IR-120 $\left(\mathrm{H}^{+}\right)$until $\mathrm{pH} 7$, filtered and evaporated. The crude was purified with prep-HPLC (20 min gradient from $10 \%$ acetonitrile $/ 90 \%$ water with $0.1 \%$ formic acid to $100 \%$ acetonitrile) to give $\mathbf{1 j}$ ( $4.5 \mathrm{mg}$, $20 \%)$.

${ }^{1} \mathrm{H}$ NMR (400 MHz, CD $\left.{ }_{3} \mathrm{OD}\right): \delta 8.46(\mathrm{~d}, J=2.3 \mathrm{~Hz}, 1 \mathrm{H}$, triazole $\mathrm{CH}), 7.91-7.85(\mathrm{~m}$, 1H), 7.63-7.57 (m, 1H), 7.53-7.41 (m, 2H), $4.48\left(\mathrm{~d}, J=9.8 \mathrm{~Hz}, \mathbf{H}^{1}\right), 4.02-3.96(\mathrm{~m}$, 2H), 3.82 (dd, J=13.0 Hz, 8.7 Hz, 1H, H') , 3.77-3.71 (m, 2H), 3.65 (dd, J=9.6 Hz, 3.3 $\mathrm{Hz}, 1 \mathrm{H}, \mathbf{H}^{3}$ )

${ }^{13} \mathrm{C}$ NMR (100 MHz, CD ${ }_{3}$ OD): $\delta 130.94,130.86,125.26,125.12,124.71,124.65$, 116.88, 116.68, 79.69, 74.86, 74.83, 70.85, 69.58, 61.52

HRMS: M+H; 326.1149 found, 326.1152 calculated. $[\alpha]^{20}{ }_{D}=21^{\circ}(c=0.16455$ in methanol $)$.

Purity by HPLC (UV/VIS detector $254 \mathrm{~nm}$ ): 99.3\% 


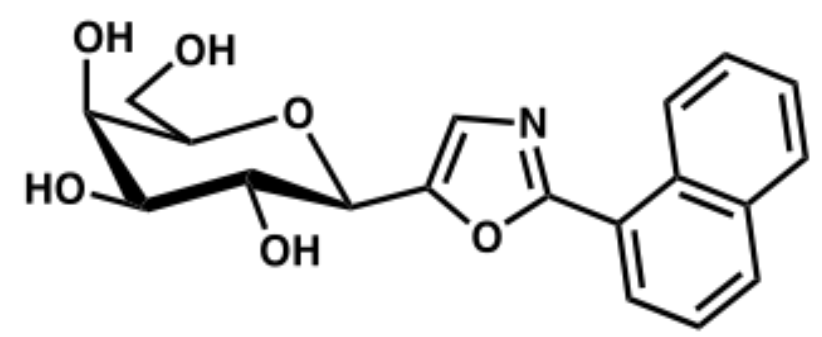

Compound $10 \mathrm{~b}$ (20 mg, $0.039 \mathrm{mmol}$ ) and of sodium methoxide (13 mg, 0.232 $\mathrm{mmol})$ were dissolved in dry methanol $(4 \mathrm{~mL})$ under nitrogen. After 3 hours, the reaction was quenched with Amberlite IR-120 ( $\left.\mathrm{H}^{+}\right)$to $\mathrm{pH} 7$, filtered and evaporated. Prep-HPLC (20 min gradient from $10 \%$ acetonitrile/ $90 \%$ water with $0.1 \%$ formic acid to $100 \%$ acetonitrile) gave $\mathbf{2 b}(7 \mathrm{mg}, 52 \%)$.

${ }^{1} \mathrm{H}$ NMR (400 MHz, CD $\left.{ }_{3} \mathrm{OD}\right): \delta 9.09-9.05(\mathrm{~m}, 1 \mathrm{H}), 8.21$ (dd, J=7.3 Hz, $1.1 \mathrm{~Hz}, 1 \mathrm{H}$ ), $8.07(\mathrm{~d}, J=8.4 \mathrm{~Hz}, 1 \mathrm{H}), 8.01-7.97(\mathrm{~m}, 1 \mathrm{H}), 7.68-7.57(\mathrm{~m}, 3 \mathrm{H}), 7.44(\mathrm{~s}, 1 \mathrm{H}$ oxazole CH), $4.45\left(\mathrm{~d}, J=9.9 \mathrm{~Hz}, 1 \mathrm{H}, \mathbf{H}^{1}\right.$ ) , $4.14\left(\mathrm{t}, J=9.6 \mathrm{~Hz} 1 \mathrm{H}, \mathbf{H}^{2}\right.$ ), 4.00 (dd J=3.2 Hz, $0.6 \mathrm{~Hz}$, $1 \mathrm{H} \mathrm{H}^{4}$ ), 3.82 (dd, $J=11.9 \mathrm{~Hz}, 8.42 \mathrm{~Hz}, 1 \mathrm{H}, \mathbf{H}^{6}$ ) 3.78-3.72 (m, 2H), 3.65 (dd, J=9.3 $\left.\mathrm{Hz}, 3.3 \mathrm{~Hz}, 1 \mathrm{H}, \mathbf{H}^{3}\right)$

${ }^{13} \mathrm{C}$ NMR (100 MHz, CD $\left.{ }_{3} \mathrm{OD}\right): \delta 150.09,131.13,128.29,127.86,127.14,126.69$, 126.081, 125.50, 124.68, 79.69, 74.89, 74.03, 69.43, 69.15, 61.43

HRMS: M+H; 358.1286 found, 358.1291 calculated. $[\alpha]^{20} \mathrm{D}=26^{\circ}(c=0.33409$ in methanol $)$.

Purity by HPLC (UV/VIS detector $254 \mathrm{~nm}$ ): 95.2\%

\section{2-Naphth-2-yl-5-(1-deoxy- $\beta$-D-galactopyranosyl)oxazole 2c}

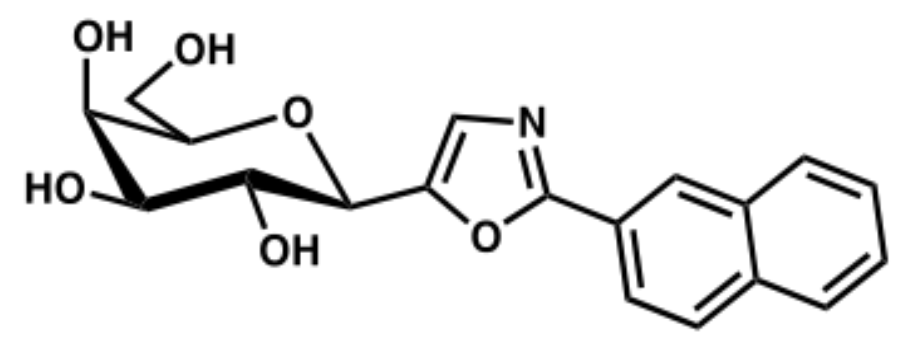

Compound 10c (30 mg, $0.057 \mathrm{mmol}$ ) and sodium methoxide (19 mg, 0.343

mmol) were dissolved in dry methanol ( $4 \mathrm{~mL})$ under nitrogen. After 3 hours, the reaction was quenched with Amberlite IR-120 $\left(\mathrm{H}^{+}\right)$to $\mathrm{pH} 7$, filtered and evaporated. Prep-HPLC (20 min gradient from $10 \%$ acetonitrile/ $90 \%$ water with $0.1 \%$ formic acid to $100 \%$ acetonitrile) gave 2 c (6 mg, 29\%).

${ }^{1} \mathrm{H}$ NMR (400 MHz, CD $\left.{ }_{3} \mathrm{OD}\right): \delta 8.61(\mathrm{~s}, 1 \mathrm{H}), 8.14(\mathrm{dd}, \mathrm{J} 1=8.5 \mathrm{~Hz}, 1.7 \mathrm{~Hz}, 1 \mathrm{H}), 8.04-$ $7.99(\mathrm{~m}, 2 \mathrm{H}), 7.97-7.93(\mathrm{~m}, 1 \mathrm{H}), 7.63-7.57(\mathrm{~m}, 2 \mathrm{H}), 7.37(\mathrm{~s}, 1 \mathrm{H}$, oxazole $\mathrm{CH}), 4.43$ (d, J=9.8 Hz, 1H, $\mathbf{H}^{1}$ ), $4.14\left(\mathrm{t}, J=9.4 \mathrm{~Hz}, 1 \mathrm{H}, \mathbf{H}^{2}\right.$ ), 4.02 (d, J=3.2 Hz, 1H, $\mathbf{H}^{6}$ ), 3.87-

$3.71(\mathrm{~m}, 3 \mathrm{H}), 3.64\left(\mathrm{dd}, J=9.4 \mathrm{~Hz}, 3.2 \mathrm{~Hz}, 1 \mathrm{H}, \mathbf{H}^{3}\right.$ )

${ }^{13} \mathrm{C}$ NMR $\left(100 \mathrm{MHz}, \mathrm{CD}_{3} \mathrm{OD}\right): \delta 150.21,134.37,133.09,128.50,128.35,127.54$, $127.31,126.81,126.71,126.15,124.18,122.65,79.67,74.84,73.99,69.41,69.03$, 61.42

HRMS: M+H; 358.1289 found, 358.1291 calculated. $[\alpha]^{20}{ }_{D}=14^{\circ}(c=0.22014$ in methanol $)$.

Purity by HPLC (UV/VIS detector $254 \mathrm{~nm}$ ): 96.6\% 


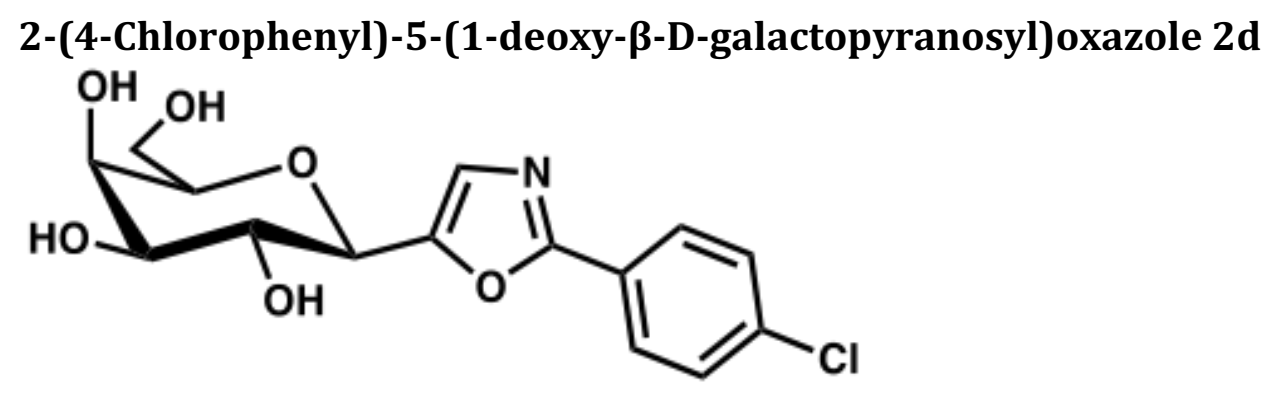

Compound 10d (10 mg, $0.019 \mathrm{mmol}$ ) and of sodium methoxide ( $6 \mathrm{mg}, 0.117$ $\mathrm{mmol}$ ) were dissolved in dry methanol (4 mL) under nitrogen. After 3 hours, the reaction was quenched with Amberlite IR- $120\left(\mathrm{H}^{+}\right)$to $\mathrm{pH} 7$, filtered and evaporated. Prep-HPLC (20 min gradient from $10 \%$ acetonitrile/90\% water with $0.1 \%$ formic acid to $100 \%$ acetonitrile) gave $\mathbf{2 d}(2 \mathrm{mg}, 30 \%$ ).

${ }^{1} \mathrm{H}$ NMR (400 MHz, $\left.\mathrm{CD}_{3} \mathrm{OD}\right): \delta 8.08-8.03(\mathrm{~m}, 2 \mathrm{H}), 7.58-7.52(\mathrm{~m}, 2 \mathrm{H}), 7.31(\mathrm{~s}, 1 \mathrm{H}$, oxazole CH), $4.38\left(\mathrm{~d}, J=9.5 \mathrm{~Hz}, 1 \mathrm{H}, \mathbf{H}^{1}\right), 4.07\left(\mathrm{t}, J=9.9 \mathrm{~Hz} 1 \mathrm{H}, \mathbf{H}^{2}\right), 3.99(\mathrm{~d} J=3.1 \mathrm{~Hz}$, $1 \mathrm{H} \mathrm{H}^{6}$ ), 3.82-3.68 (m, 3H), 3.61 (dd, J=9.4 Hz, $3.4 \mathrm{~Hz}, 1 \mathrm{H}, \mathbf{H}^{3}$ )

${ }^{13} \mathrm{C}$ NMR $\left(100 \mathrm{MHz}, \mathrm{CD}_{3} \mathrm{OD}\right): \delta 128.91,127.51,126.82,79.64,74.79,73.90,69.36$, $69.01,61.39$

HRMS: $\mathrm{M}+\mathrm{H} ; 342.0743$ found, 342.0744 calculated.

$[\alpha]^{20}{ }_{D}=18^{\circ}(c=0.14658$ in methanol $)$.

Purity by HPLC (UV/VIS detector $254 \mathrm{~nm}$ ): 97.1\%

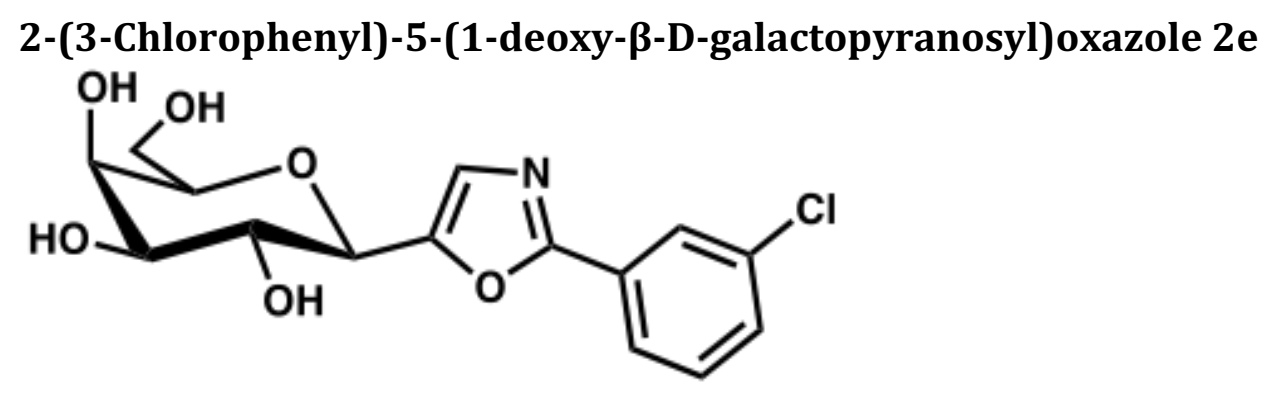

Compound 10e (10 mg, $0.019 \mathrm{mmol}$ ) and sodium methoxide ( $6 \mathrm{mg}, 0.117 \mathrm{mmol})$ were dissolved in dry methanol (4 mL) under nitrogen. After 3 hours, the reaction was quenched with Amberlite IR-120 $\left(\mathrm{H}^{+}\right)$to $\mathrm{pH} 7$, filtered and evaporated. Prep-HPLC (20 min gradient from $10 \%$ acetonitrile/90\% water with $0.1 \%$ formic acid to $100 \%$ acetonitrile) gave $2 \mathbf{e}(2 \mathrm{mg}, 30 \%)$.

${ }^{1} \mathrm{H}$ NMR (400 MHz, $\left.\mathrm{CD}_{3} \mathrm{OD}\right): \delta 8.10-8.07(\mathrm{~m}, 1 \mathrm{H}), 8.02-7.98(\mathrm{~m}, 1 \mathrm{H}), 7.56-7.49(\mathrm{~m}$, $2 \mathrm{H}), 7.33\left(\mathrm{~s}, 1 \mathrm{H}\right.$, oxazole CH), $4.39\left(\mathrm{~d}, J=9.8 \mathrm{~Hz}, 1 \mathrm{H}, \mathbf{H}^{1}\right), 4.08\left(\mathrm{t}, J=9.8 \mathrm{~Hz} 1 \mathrm{H}, \mathbf{H}^{2}\right)$, 3.99 (d J=3.4 Hz, $1 \mathrm{H} \mathrm{H}^{6}$ ), 3.83-3.69 (m, 3H), 3.61 (dd, J=9.5 Hz, $3.4 \mathrm{~Hz}, 1 \mathrm{H}, \mathbf{H}^{3}$ ) ${ }^{13} \mathrm{C}$ NMR (100 MHz, CD $\left.{ }_{3} \mathrm{OD}\right): \delta 150.56,134.68,130.33,128.81,126.94,125.85$, $124.26,79.68,74.79,73.90,69.40,68.98,61.41$

HRMS: $\mathrm{M}+\mathrm{H} ; 342.0744$ found, 342.0744 calculated. $[\alpha]^{20}{ }_{D}=12^{\circ}(c=0.09179$ in methanol $)$.

Purity by HPLC (UV/VIS detector $254 \mathrm{~nm}$ ): 99.5\% 


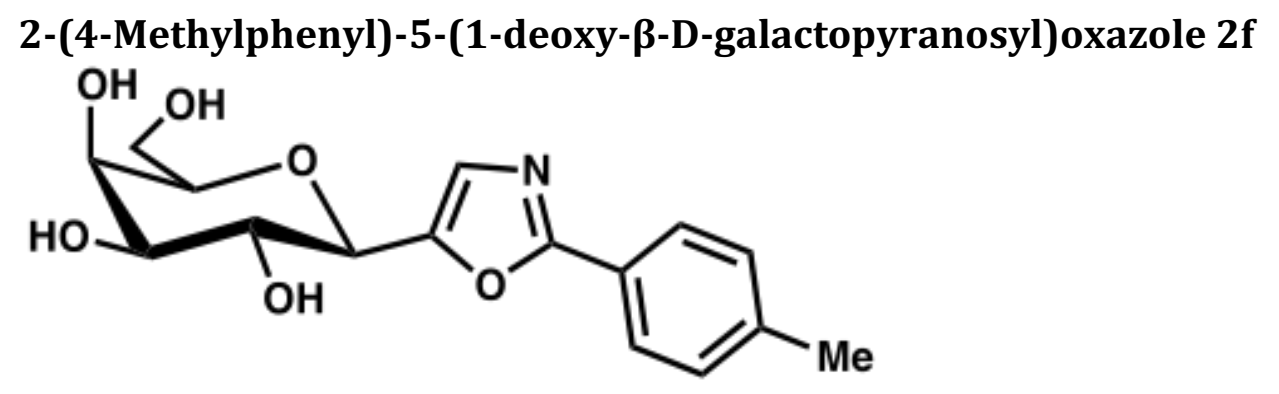

Compound 10f ( $3.3 \mathrm{mg}, 0.007 \mathrm{mmol}$ ) and sodium methoxide ( $2 \mathrm{mg}, 0.040 \mathrm{mmol}$ ) were dissolved inof dry methanol $(2 \mathrm{~mL})$ under nitrogen. After 3 hours, the reaction was quenched with Amberlite IR-120 $\left(\mathrm{H}^{+}\right)$to $\mathrm{pH} 7$, filtered and evaporated. Prep-HPLC (20 min gradient from $10 \%$ acetonitrile/ $90 \%$ water with $0.1 \%$ formic acid to $100 \%$ acetonitrile) gave $\mathbf{2 f}(1 \mathrm{mg}, 46 \%$ ).

${ }^{1} \mathrm{H}$ NMR (400 MHz, $\left.\mathrm{CD}_{3} \mathrm{OD}\right)$ : $\delta$ 7.97-7-93 (m, 2H), 7.37-7.32 (m, 2H), $7.28(\mathrm{~s}, 1 \mathrm{H}$, oxazole $\mathrm{CH}$ ), $4.37\left(\mathrm{~d}, J=9.8 \mathrm{~Hz}, 1 \mathrm{H}, \mathbf{H}^{1}\right), 4.08\left(\mathrm{t}, J=9.5 \mathrm{~Hz} 1 \mathrm{H}, \mathbf{H}^{2}\right), 3.99$ (d J=3.3 Hz, $\left.1 \mathrm{H} \mathrm{H}^{6}\right), 3.83-3.68(\mathrm{~m}, 3 \mathrm{H}), 3.61\left(\mathrm{dd}, J=9.5 \mathrm{~Hz}, 3.6 \mathrm{~Hz}, 1 \mathrm{H}, \mathrm{H}^{3}\right), 2.45$ (s, 3H, phenyl methyl)

${ }^{13} \mathrm{C}$ NMR $\left(100 \mathrm{MHz}, \mathrm{CD}_{3} \mathrm{OD}\right): \delta$ 129.26, $126.42,126.03,79.63,74.85,73.95,69.40$, $68.97,61.41,20.07$

HRMS: M+H; 322.1293 found, 322.1291 calculated.

$[\alpha]^{20} \mathrm{D}=16^{\circ}(\mathrm{c}=0.005289$ in methanol $)$.

Purity by HPLC (UV/VIS detector $254 \mathrm{~nm}$ ): 99.1\%

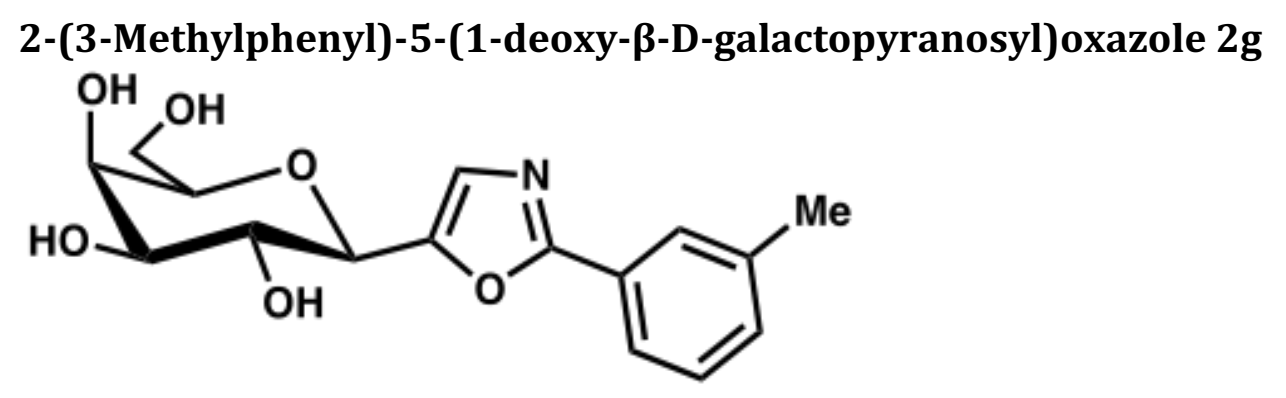

Compound $10 \mathrm{~g}$ (22 $\mathrm{mg}, 0.045 \mathrm{mmol}$ ) and sodium methoxide (15 mg, 0.270 $\mathrm{mmol}$ ) were dissolved in dry methanol (4 mL) under nitrogen. After 3 hours, the reaction was quenched with Amberlite IR- $120\left(\mathrm{H}^{+}\right)$to $\mathrm{pH} 7$, filtered and evaporated. Prep-HPLC (20 min gradient from $10 \%$ acetonitrile/90\% water with $0.1 \%$ formic acid to $100 \%$ acetonitrile) gave $2 \mathrm{~g}$ (7 $\mathrm{mg}, 46 \%)$.

${ }^{1} \mathrm{H}$ NMR $\left(400 \mathrm{MHz}, \mathrm{CD}_{3} \mathrm{OD}\right): \delta 7.90(\mathrm{~s}, 1 \mathrm{H}), 7.85(\mathrm{~d}, J=7.7 \mathrm{~Hz}, 1 \mathrm{H}), 7.40(\mathrm{t}, J=7.6$ $\mathrm{Hz}, 1 \mathrm{H}), 7.35(\mathrm{~d}, J=7.7 \mathrm{~Hz}, 1 \mathrm{H}), 4.38\left(\mathrm{~d}, J=9.7 \mathrm{~Hz}, 1 \mathrm{H}, \mathbf{H}^{1}\right), 4.09\left(\mathrm{t}, J=9.7 \mathrm{~Hz} 1 \mathrm{H}, \mathbf{H}^{2}\right)$, 3.99 (d J=3.4 Hz, $1 \mathrm{H} \mathrm{H^{6 }}$ ), 3.83-3.68 (m, 3H), 3.61 (dd, J=9.3 Hz, $3.5 \mathrm{~Hz}, 1 \mathrm{H}, \mathbf{H}^{3}$ ), 2.44 (s, 3H, phenyl methyl)

${ }^{13} \mathrm{C}$ NMR (100 MHz, CD $\left.{ }_{3} \mathrm{OD}\right): \delta 149.94,138.68,131.23,128.56,126.84,126.52$, 126.50, 123.21, 79.64, 74.84, 73.95, 69.40, 68.98, 61.41, 19.95

HRMS: $\mathrm{M}+\mathrm{H} ; 322.1288$ found, 322.1291 calculated. $[\alpha]^{20}{ }_{D}=17^{\circ}(c=0.21697$ in methanol $)$.

Purity by HPLC (UV/VIS detector $254 \mathrm{~nm}$ ): 99.5\% 


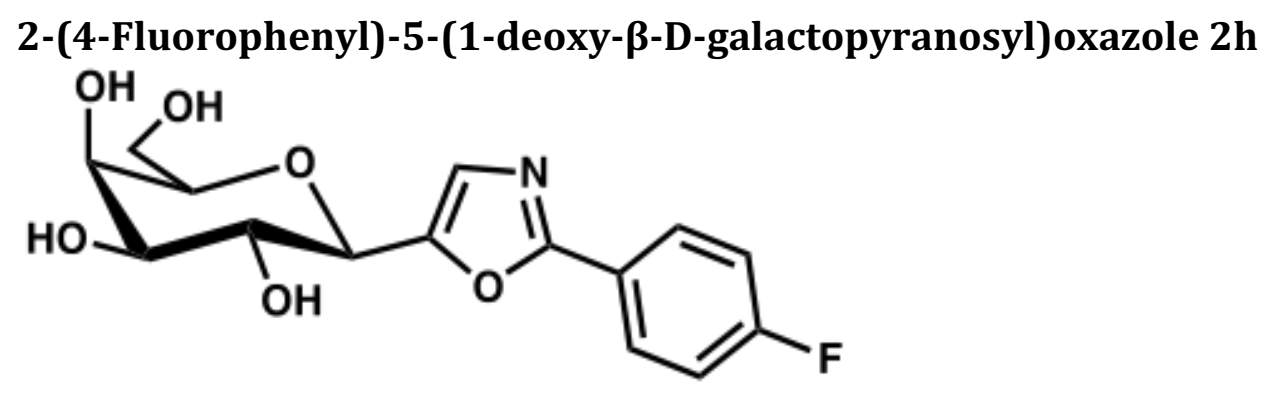

Compound $2 \mathrm{~h}$ ( $7 \mathrm{mg}, 0.014 \mathrm{mmol})$ and sodium methoxide ( $5 \mathrm{mg}, 0.085 \mathrm{mmol})$ were dissolved in dry methanol ( $2 \mathrm{~mL})$ under nitrogen. After 3 hours, the reaction was quenched with Amberlite IR-120 $\left(\mathrm{H}^{+}\right)$to $\mathrm{pH}$ 7, filtered and evaporated. Prep-HPLC (20 min gradient from $10 \%$ acetonitrile/ $90 \%$ water with $0.1 \%$ formic acid to $100 \%$ acetonitrile) gave $\mathbf{2 h}(2 \mathrm{mg}, 43 \%)$.

${ }^{1} \mathrm{H}$ NMR (400 MHz, $\left.\mathrm{CD}_{3} \mathrm{OD}\right): \delta 8.14-8.07(\mathrm{~m}, 2 \mathrm{H}), 7.32-7.23(\mathrm{~m}, 3 \mathrm{H}) 4.37$ (d, $J=$ $\left.10.0 \mathrm{~Hz}, 1 \mathrm{H}, \mathbf{H}^{1}\right), 4.08\left(\mathrm{t}, J=8.9 \mathrm{~Hz} 1 \mathrm{H}, \mathbf{H}^{2}\right), 3.98\left(\mathrm{~d} J=3.3 \mathrm{~Hz}, 1 \mathrm{H} \mathbf{H}^{6}\right), 3.83-3.68(\mathrm{~m}$, $3 \mathrm{H}), 3.61$ (dd, $J=9.4 \mathrm{~Hz}, 3.4 \mathrm{~Hz}, 1 \mathrm{H}, \mathbf{H}^{3}$ )

${ }^{13} \mathrm{C}$ NMR (100 MHz, CD $\left.{ }_{3} \mathrm{OD}\right): \delta 128.46,128.38,126.64,115.79,115.56,79.63$, $74.82,73.91,69.38,68.98,61.40$

HRMS: $\mathrm{M}+\mathrm{H} ; 326.1035$ found, 326.1040 calculated.

$[\alpha]^{20}{ }_{D}=18^{\circ}(c=0.07776$ in methanol $)$.

Purity by HPLC (UV/VIS detector $254 \mathrm{~nm}$ ): 96.6\%

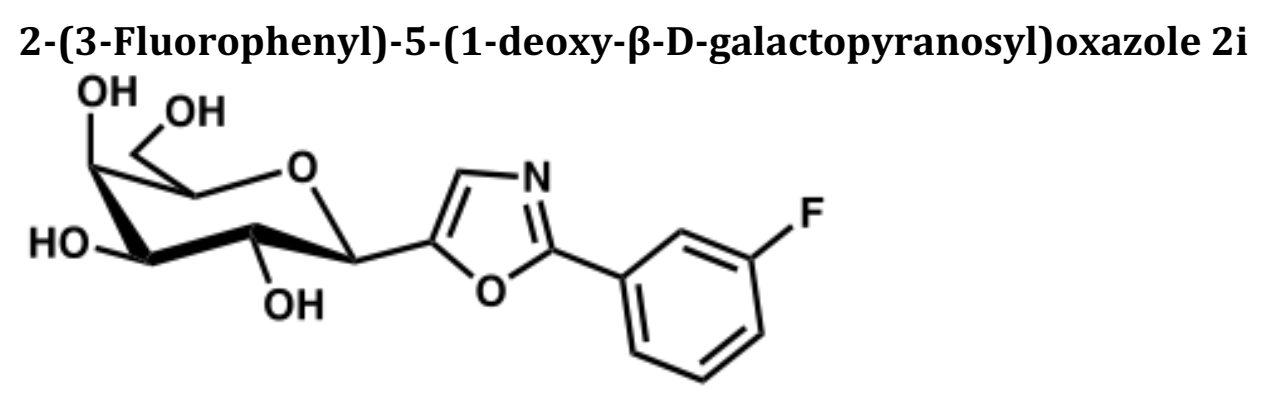

Compound 10i $(10 \mathrm{mg}, 0.020 \mathrm{mmol})$ and sodium methoxide $(7 \mathrm{mg}, 0.122 \mathrm{mmol})$ were dissolved in dry methanol ( $2 \mathrm{~mL})$ under nitrogen. After 3 hours, the reaction was quenched with Amberlite IR-120 $\left(\mathrm{H}^{+}\right)$to $\mathrm{pH} 7$, filtered and evaporated. Prep-HPLC (20 min gradient from $10 \%$ acetonitrile/ $90 \%$ water with $0.1 \%$ formic acid to $100 \%$ acetonitrile) gave $2 \mathbf{i}(1 \mathrm{mg}, 18 \%)$.

${ }^{1} \mathrm{H}$ NMR ( $400 \mathrm{MHz}, \mathrm{CD}_{3} \mathrm{OD}$ ): $\delta 7.90$ (ddd, $J=7.8 \mathrm{~Hz}, 1.7 \mathrm{~Hz}, 0.9 \mathrm{~Hz}, 1 \mathrm{H}$ ), 7.79 (ddd, $J=9.8 \mathrm{~Hz}, 2.6 \mathrm{~Hz}, 1.5 \mathrm{~Hz}$ ), 7.59-7.52 (m, 1H), $7.33(\mathrm{~s}, 1 \mathrm{H}$, oxazole $\mathrm{CH}), 7.28$ (tdd, $J=$ $8.5 \mathrm{~Hz}, 2.7 \mathrm{~Hz}, 0.8 \mathrm{~Hz}, 1 \mathrm{H}), 4.39$ (d, J= $\left.9.8 \mathrm{~Hz}, 1 \mathrm{H}, \mathbf{H}^{1}\right), 4.08\left(\mathrm{t}, J=8.4 \mathrm{~Hz} 1 \mathrm{H}, \mathbf{H}^{2}\right)$, 3.99 (d J=3.3 Hz, $1 \mathrm{H} \mathrm{H}^{6}$ ), 3.83-3.68 (m, 3H), 3.61 (dd, J=9.4 Hz, $3.3 \mathrm{~Hz}, 1 \mathrm{H}, \mathbf{H}^{3}$ ) ${ }^{13} \mathrm{C}$ NMR (100 MHz, CD $\left.30 D\right): \delta 130.79,126.89,121.92,117.31,79.67,74.80$, 73.90, 69.38, 69.01, 61.40

HRMS: $\mathrm{M}+\mathrm{H}$; 326.1035 found, 326.1040 calculated. $[\alpha]^{20}{ }_{D}=19^{\circ}(c=0.06491$ in methanol $)$.

Purity by HPLC (UV/VIS detector $254 \mathrm{~nm}$ ): 99.2\% 


\section{3-Naphth-1-yl-5-(1-deoxy- $\beta$-D-galactopyranosyl)isoxazole $3 b$}

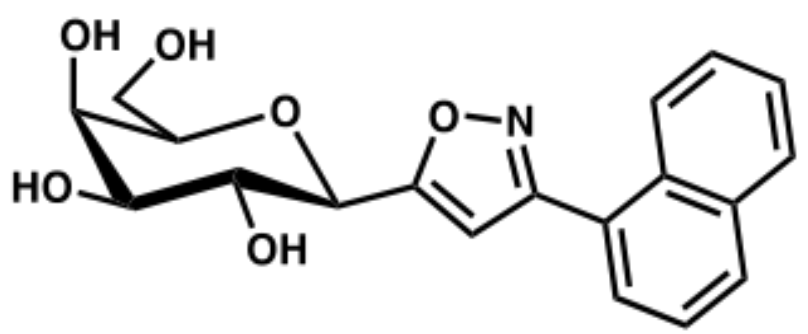

Compound 9b (84 mg, $0.165 \mathrm{mmol}$ ) was dissolved in dry THF ( $3 \mathrm{~mL}$ ) with hydroxylamine hydrochloride (29 $\mathrm{mg}, 0.411 \mathrm{mmol}$ ) and of sodium carbonate (71 $\mathrm{mg}, 0.666 \mathrm{mmol}$ ) and refluxed overnight under nitrogen. The reaction was poured into ethyl acetate $(30 \mathrm{~mL})$ and washed with brine. The brine was extracted two times with ethyl acetate $(30 \mathrm{~mL})$, the organic phases were pooled, dried with anhydrous sodium sulfate and evaporated. The crude was dissolved in dry methanol ( $2 \mathrm{~mL}$ ) with of sodium methoxide (39 $\mathrm{mg}, 0.708 \mathrm{mmol}$ ). After 3 hours, the reaction was quenched by addition of Amberlite IR-120 $\left(\mathrm{H}^{+}\right)$until pH 7 , filtered \& evaporated. The crude was purified first with column chromatography (5:1 dichloromethane/methanol) and then with prep-HPLC to give $\mathbf{3 b}$ (11 mg, 11\%).

${ }^{1} \mathrm{H}$ NMR (400 MHz, CD $\left.{ }_{3} \mathrm{OD}\right): \delta 8.36-8.31(\mathrm{~m}, 1 \mathrm{H}), 8.06(\mathrm{~d}, J=8.2 \mathrm{~Hz}, 1 \mathrm{H}), 8.03-7.98$ $(\mathrm{m}, 1 \mathrm{H}), 7.89-7.84(\mathrm{~m}, 1 \mathrm{H}), 7.62(3 \mathrm{H}, \mathrm{m}), 7.05(\mathrm{~s}, 1 \mathrm{H}$ isoxazole $\mathrm{CH}), 4.37(\mathrm{~d}, J=$ $\left.10.0 \mathrm{~Hz}, 1 \mathrm{H}, \mathbf{H}^{1}\right), 4.01\left(\mathrm{~d}, J=3.1 \mathrm{~Hz}, 1 \mathrm{H}, \mathbf{H}^{4}\right), 3.89\left(\mathrm{t}, J=9.4 \mathrm{~Hz} 1 \mathrm{H}, \mathbf{H}^{2}\right), 3.81(\mathrm{dd}$ $\left.J=10.0 \mathrm{~Hz}, 4.3 \mathrm{~Hz} 1 \mathrm{H} \mathrm{H}^{5}\right), 3.77-3.68(\mathrm{~m}, 2 \mathrm{H}), 3.64\left(\mathrm{dd}, J=9.4 \mathrm{~Hz}, 3.3 \mathrm{~Hz}, 1 \mathrm{H}, \mathbf{H}^{3}\right.$ ) ${ }^{13} \mathrm{C}$ NMR $\left(100 \mathrm{MHz}, \mathrm{CD}_{3} \mathrm{OD}\right): \delta 169.75,163.40,133.96,130.67,130.10,128.44$, $127.28,127.08,126.17,124.90,124.44,102.37,79.72,75.13,74.65,70.11,69.42$, 61.45

HRMS: $\mathrm{M}+\mathrm{Na} ; 380.1115$ found, 380.1110 calculated. $[\alpha]^{20}{ }_{D}=21^{\circ}(c=0.23211$ in methanol $)$.

Purity by HPLC (UV/VIS detector $254 \mathrm{~nm}$ ): 99.3\%

\section{3-Naphth-2-yl-5-(1-deoxy- $\beta$-D-galactopyranosyl)isoxazole 3c}

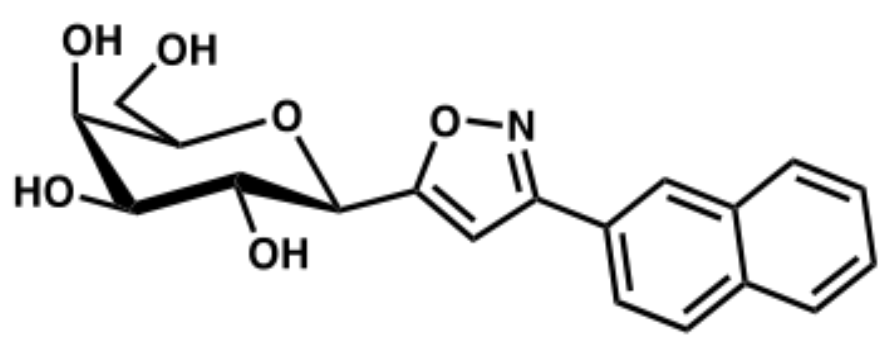

Compound 9c (79 mg, $0.155 \mathrm{mmol}$ ) was dissolved in dry THF ( $3 \mathrm{~mL}$ ) with hydroxylamine hydrochloride (27 $\mathrm{mg}, 0.388 \mathrm{mmol}$ ) and sodium carbonate (30 $\mathrm{mg}$, $0.620 \mathrm{mmol}$ ) and refluxed overnight under nitrogen. The reaction was poured into ethyl acetate $(30 \mathrm{~mL})$ and washed with brine. The brine was extracted two times with ethyl acetate $(30 \mathrm{~mL})$, the organic phases were pooled, dried with anhydrous sodium sulfate and evaporated. The crude was dissolved in dry methanol ( $2 \mathrm{~mL}$ ) with of sodium methoxide $(16 \mathrm{mg}, 0.422 \mathrm{mmol})$. After 3 hours the reaction was quenched by addition of Amberlite IR-120 $\left(\mathrm{H}^{+}\right)$until $\mathrm{pH} 7$, filtered \& evaporated. The crude was purified first with column chromatography (5:1 dichloromethane/methanol) and then with prep-HPLC to give 3c (17 mg, 40\%). 
${ }^{1} \mathrm{H}$ NMR (400 MHz, CD $\left.{ }_{3} \mathrm{OD}\right): \delta 8.41(\mathrm{~d}, J=1.2 \mathrm{~Hz}, 1 \mathrm{H}), 8.04-8.00(\mathrm{~m}, 2 \mathrm{H}), 7.95-7.91$ $(\mathrm{m}, 2 \mathrm{H}), 7.61-7.57(\mathrm{~m}, 2 \mathrm{H}) 7.11(\mathrm{~s}, 1 \mathrm{H}$ isoxazole $\mathrm{CH}), 4.37\left(\mathrm{~d}, J=10.0 \mathrm{~Hz}, 1 \mathrm{H}, \mathbf{H}^{\mathbf{1}}\right)$, $4.01\left(\mathrm{~d}, J=3.1 \mathrm{~Hz}, 1 \mathrm{H}, \mathbf{H}^{4}\right), 3.89$ (t, J=9.4 Hz 1H, $\mathbf{H}^{2}$ ), 3.81 (dd $J=10.0 \mathrm{~Hz}, 4.3 \mathrm{~Hz} 1 \mathrm{H}$ $\mathbf{H}^{5}$ ), 3.77-3.68 (m, 2H), $3.64\left(\mathrm{dd}, J=9.4 \mathrm{~Hz}, 3.2 \mathrm{~Hz}, 1 \mathrm{H}, \mathbf{H}^{3}\right.$ )

${ }^{13} \mathrm{C}$ NMR (100 MHz, CD ${ }_{3}$ OD): $\delta 128.68,128.31,127.51,127.12,126.69,124.98$, $122.40,98.74,79.72,75.13,74.65,70.11,69.42,61.45$

HRMS: M+H; 358.1292 found 358.1291 calculated $[\alpha]^{20}{ }_{D}=21^{\circ}(c=0.22913$ in methanol $)$.

Purity by HPLC (UV/VIS detector $254 \mathrm{~nm}$ ): 96.6\%

\section{3-Naphth-1-yl-5-(1-deoxy- $\beta$-D-galactopyranosyl)-1H-pyrazole 4b}

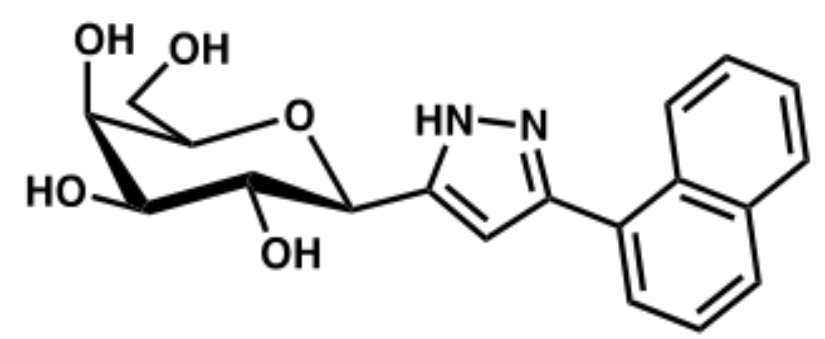

Compound 9b (137 mg, $0.269 \mathrm{mmol}$ ) was dissolved in THF (6 mL) and sodium carbonate (120 mg, $1.128 \mathrm{mmol})$ and hydrazine hydrate $(130 \mu \mathrm{L}, 1.694 \mathrm{mmol}$, $64 \%$ ) were added. The reaction mixture was refluxed overnight, cooled to room temperature, filtrated and evaporated. Column chromatography (5:1 dichloromethane/methanol) followed by prep-HPLC (20 min gradient from $10 \%$ acetonitrile/ $90 \%$ water with $0.1 \%$ formic acid to $100 \%$ acetonitrile) gave $\mathbf{4 b}$ ( $74 \mathrm{mg}$, $77 \%)$.

${ }^{1} \mathrm{H}$ NMR (400 MHz, CD $\left.{ }_{3} \mathrm{OD}\right): \delta 8.31-8.25(\mathrm{~m}, 1 \mathrm{H}), 7.96-7.89(\mathrm{~m}, 2 \mathrm{H}), 7.62(\mathrm{dd}$, $J=7.2, \mathrm{~Hz}, 1.2 \mathrm{~Hz}, 1 \mathrm{H}), 7.57-7.47(\mathrm{~m}, 3 \mathrm{H}) 6.77(\mathrm{~s}, 1 \mathrm{H}$ pyrazole $\mathrm{CH}), 4.33(\mathrm{~d}, J=9.9$ $\mathrm{Hz}, 1 \mathrm{H}, \mathbf{H}^{1}$ ), $3.98\left(\mathrm{~d}, J=3.0 \mathrm{~Hz}, 1 \mathrm{H}, \mathbf{H}^{4}\right.$ ), 3.90 (t, J=9.6 Hz 1H, $\mathbf{H}^{2}$ ), 3.83 (dd J=11.2 $\mathrm{Hz}, 7.1 \mathrm{~Hz}, 1 \mathrm{H} \mathrm{H}^{5}$ ), 3.77-3.68 (m, 2H), 3.61 (dd, $J=9.0 \mathrm{~Hz}, 3.3 \mathrm{~Hz}, 1 \mathrm{H}, \mathbf{H}^{3}$ ) ${ }^{13} \mathrm{C}$ NMR (100 MHz, CD ${ }_{3}$ OD): $\delta 146.76,133.96,131.36,129.87,128.37,128.00$, 126.77, 125.66, 125.28, 124.93, 101.12, 79.45, 75.70, 74.89, 70.95, 69.56, 61.57 HRMS: $\mathrm{M}+\mathrm{H}$; 357.1459 found, 357.1450 calculated. $[\alpha]^{20}{ }_{D}=19^{\circ}(c=0.22521$ in methanol $)$. Purity by HPLC (UV/VIS detector $254 \mathrm{~nm}$ ): 97.3\%

\section{3-Naphth-2-yl-5-(1-deoxy- $\beta$-D-galactopyranosyl)-1H-pyrazole 4c}

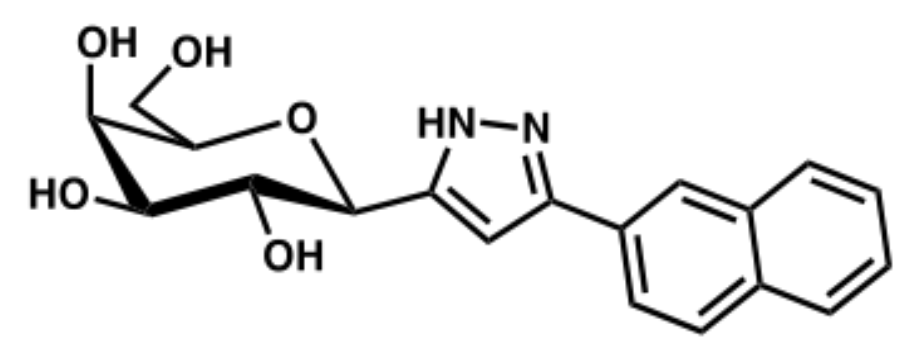

Compound 9c (146 mg, $0.286 \mathrm{mmol})$ was dissolved in THF (6 mL) and sodium carbonate (76 mg, $0.715 \mathrm{mmol})$ and hydrazine hydrate $(32 \mu \mathrm{L}, 0.429 \mathrm{mmol}$, $64 \%$ ) were added. The reaction mixture was refluxed overnight, poured into ethyl acetate $(30 \mathrm{~mL})$, washed with brine $(30 \mathrm{~mL})$ which was extracted twice with ethyl acetate $(30 \mathrm{~mL})$. The organic phases were pooled, dried with sodium sulfate 
and evaporated. The crude was dissolved in dry methanol ( $6 \mathrm{~mL})$ with sodium methoxide (187 mg, $3.46 \mathrm{mmol}$ ) under nitrogen and left for 4 hours. Upon completion, the reaction was quenched with Amberlite IR-120 $\left(\mathrm{H}^{+}\right)$until pH 7, filtered and evaporated. Column chromatography (5:1 dichloromethane/methanol) followed by prep-HPLC (20 min gradient from 10\% acetonitrile/90\% water with $0.1 \%$ formic acid to $100 \%$ acetonitrile) gave $4 \mathrm{c}$ (90 $\mathrm{mg}, 88 \%$ ).

${ }^{1} \mathrm{H}$ NMR (400 MHz, $\left.\mathrm{CD}_{3} \mathrm{OD}\right): \delta 8.24(\mathrm{~s}, 1 \mathrm{H}), 7.99-7.80(\mathrm{~m}, 4 \mathrm{H}), 7.55-7.44(\mathrm{~m}, 2 \mathrm{H})$, $6.92(\mathrm{~s}, 1 \mathrm{H}$ pyrazole $\mathrm{CH}), 4.33\left(\mathrm{~d}, J=9.9 \mathrm{~Hz}, 1 \mathrm{H}, \mathbf{H}^{1}\right), 3.98\left(\mathrm{~d}, J=3.0 \mathrm{~Hz}, 1 \mathrm{H}, \mathbf{H}^{4}\right.$ ),

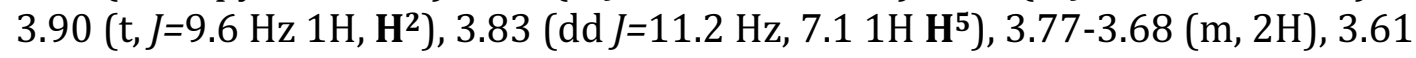
(dd, J=9.0 Hz, $3.3 \mathrm{~Hz}, 1 \mathrm{H}, \mathbf{H}^{3}$ )

${ }^{13} \mathrm{C}$ NMR $\left(100 \mathrm{MHz}, \mathrm{CD}_{3} \mathrm{OD}\right): \delta 133.63,133.13,127.75,127.33,123.78,101.44$, 79.45, 75.70, 74.89, 70.95, 69.56, 61.57

HRMS: $\mathrm{M}+\mathrm{H}$; 357.1457 found, 157.1450 calculated.

$[\alpha]^{20}{ }_{D}=23^{\circ}(c=0.42031$ in methanol $)$.

Purity by HPLC (UV/VIS detector $254 \mathrm{~nm}$ ): 97.3\%

\section{1-Naphth-1-yl-3-(2,3,4,6-tetra-O-acetyl- $\beta$-D-galactopyranosyl)-prop-2-yn-} 1-one $9 b$

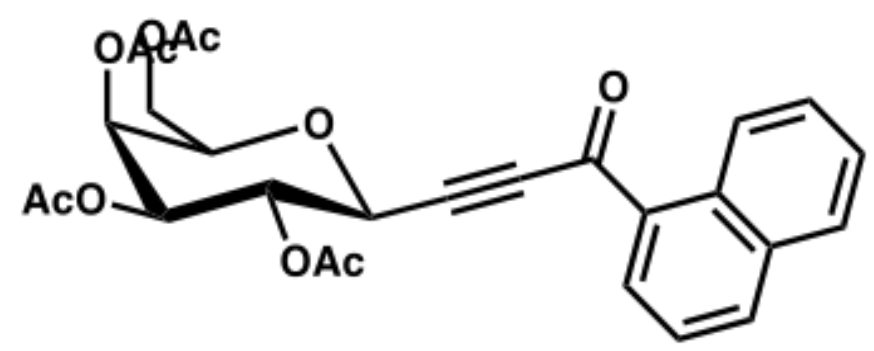

Compound 8 (150 mg, $0.421 \mathrm{mmol}$ ), 1-naphthoyl chloride ( $85 \mathrm{mg}, 0.463 \mathrm{mmol}$ ), bis(triphenylphosphine) palladium(II) dichloride (15 $\mathrm{mg}, 0.021 \mathrm{mmol}$ ) and copper(I) iodide (12 mg, $0.063 \mathrm{mmol}$ ) were dissolved in dry THF ( $5 \mathrm{~mL}$ ) and triethylamine $(0.120 \mathrm{~mL}, 0.842 \mathrm{mmol})$ was added. The reaction mixture goes from pale yellow to a deep orange upon triethylamine addition. After $15 \mathrm{mins}$, the reaction mixture was poured into ethyl acetate $(40 \mathrm{~mL})$, washed with brine (40 $\mathrm{mL})$, the brine was extracted twice with ethyl acetate $(40 \mathrm{~mL})$, the organic phases pooled, dried with sodium sulfate and evaporated. Column chromatography (2:1 heptane/ethyl acetate) gave $9 \mathbf{b}$ (137 $\mathrm{mg}, 64 \%)$.

${ }^{1} \mathrm{H} \mathrm{NMR}\left(400 \mathrm{MHz} \mathrm{CDCl}_{3}\right.$ ): $\delta 9.19(\mathrm{dd}, \mathrm{J} 1=8.5 \mathrm{~Hz}, \mathrm{~J} 2=0.8 \mathrm{~Hz}, 1 \mathrm{H}), 8.53$ (dd, J= 7.3 $\mathrm{Hz}, 1.3 \mathrm{~Hz}, 1 \mathrm{H}), 8.13$ (d, J=8.2 Hz, 1H), 7.96-7.91 (m, 1H), 7.73-7.67 (m, 1H), 7.65$7.58(\mathrm{~m}, 2 \mathrm{H}), 5.59\left(\mathrm{t}, J=10.1 \mathrm{~Hz}, 1 \mathrm{H}, \mathbf{H}^{2}\right), 5.50\left(\mathrm{dd}, J=3.4 \mathrm{~Hz}, 1.0 \mathrm{~Hz}, 1 \mathrm{H}, \mathbf{H}^{4}\right), 5.11$ (dd, J=10.2 Hz, 3.3 Hz, 1H, H³), 4.51 (d, J=10.1 Hz, 1H, H' ${ }^{1}$ ), 4.22-4.17 (m, 2H), 4.04-3.99 (m, 1H, H5), $2.22(\mathrm{~s}, 3 \mathrm{H}), 2.14(\mathrm{~s}, 3 \mathrm{H}), 2.11(\mathrm{~s}, 3 \mathrm{H}), 2.04(\mathrm{~s}, 3 \mathrm{H})$ ${ }^{13} \mathrm{C}$ NMR $\left(100 \mathrm{MHz}, \mathrm{CDCl}_{3}\right): \delta 135.78,135.59,129.31,128.66,126.95,125.86$, $124.48,85.04,74.99,71.41,69.17,67.77,67.26,61.59,20.71,20.62$ $[\alpha]^{20}{ }_{D}=-12^{\circ}(c=0.59553$ in chloroform $)$. HRMS: M+H; 511.1601 found, 511.1604 calculated. 


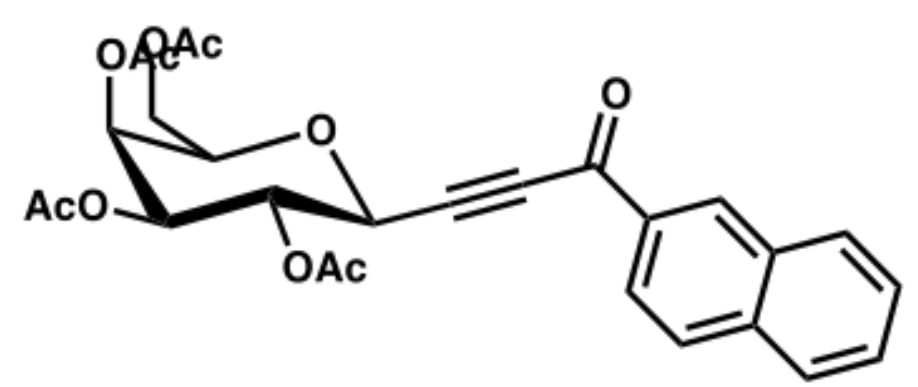

Compound 8 (150 mg, $0.421 \mathrm{mmol}$ ), 2-naphthoyl chloride ( $88 \mathrm{mg}, 0.463 \mathrm{mmol}$ ), bis(triphenylphosphine) palladium(II) dichloride (15 mg, $0.021 \mathrm{mmol}$ ) and copper(I) iodide (12 mg, $0.063 \mathrm{mmol}$ ) were dissolved in dry THF ( $5 \mathrm{~mL}$ ) and triethylamine $(0.120 \mathrm{~mL}, 0.842 \mathrm{mmol})$ was added. The reaction mixture goes from pale yellow to a deep orange upon triethylamine addition. After 1 hour, the reaction mixture was poured into ethyl acetate $(40 \mathrm{~mL})$, washed with brine $(40 \mathrm{~mL})$, the brine was extracted twice with ethyl acetate $(40 \mathrm{~mL})$, the organic phases pooled, dried with sodium sulfate and evaporated. Column chromatography (1.5:1 heptane/ethyl acetate) gave 9c (146 mg, 68\%).

${ }^{1} \mathrm{H}$ NMR $\left(400 \mathrm{MHz}, \mathrm{CDCl}_{3}\right): \delta 8.71(\mathrm{~s}, 1 \mathrm{H}), 8.16-8.08(\mathrm{~m}, 2 \mathrm{H}), 7.95-7.90(\mathrm{~m}, 2 \mathrm{H})$, 7.71-7.60 (m, 2H), $5.65\left(\mathrm{t}, J=10.2 \mathrm{~Hz}, 1 \mathrm{H}, \mathbf{H}^{2}\right), 5.52\left(\mathrm{dd}, J=3.4 \mathrm{~Hz}, 1.0 \mathrm{~Hz}, 1 \mathrm{H}, \mathbf{H}^{4}\right.$ ),

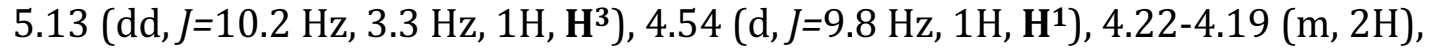
4.04-3.99 (m, 1H, $\left.\mathbf{H}^{5}\right), 2.23(\mathrm{~s}, 3 \mathrm{H}), 2.15(\mathrm{~s}, 3 \mathrm{H}), 2.11(\mathrm{~s}, 3 \mathrm{H}), 2.05(\mathrm{~s}, 3 \mathrm{H})$ ${ }^{13} \mathrm{C}$ NMR $\left(100 \mathrm{MHz}, \mathrm{CDCl}_{3}\right): \delta 176.82,170.48,170.22,170.06,169.56,136.40$, $133.59,132.51,129.99,129.37,128.69,127.94,127.12,123.46,86.09,83.64$, 75.07, 71.41, 69.13, 67.79, 67.26, 61.60, 20.79, 20.75, 20.72, 20.62 $[\alpha]^{20} \mathrm{D}=-7^{\circ}(c=0.67860$ in chloroform $)$. HRMS: M+H; 511.1612 found, 511.1604 calculated.

2-Naphth-1-yl-5-(2,3,4,6-tetra-O-acetyl-1-deoxy- $\beta$-D-galactopyranosyl)oxazole 10b

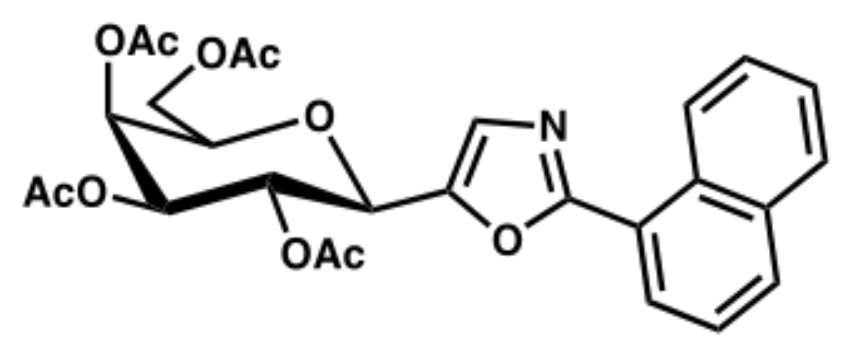

Compound 8 (100 mg, $0.281 \mathrm{mmol}$ ), 8-methylquinoline N-oxide (67 mg, 0.421 mmol), [bis(trifluoromethanesulfonyl)imidate](triphenylphosphine)gold(I) 2:1 toluene adduct (22 mg, $0.028 \mathrm{mmol}$ ) and 1-cyanonaphthalene (150 mg, 0.980 mmol) were mixed, and heated to $75^{\circ} \mathrm{C}$ where the mixture melted. The reaction was left under nitrogen for 48 hours, then diluted with ethyl acetate $(20 \mathrm{~mL})$ and washed with brine $(20 \mathrm{~mL})$. The brine was extracted two times with ethyl acetate $(20 \mathrm{~mL})$, the organic phases were pooled, dried with anhydrous sodium sulfate and evaporated. Column chromatography (1.5:1 heptane/ethyl acetate) gave 10b (49 mg, 33\%). 
${ }^{1} \mathrm{H} \mathrm{NMR}\left(400 \mathrm{MHz}, \mathrm{CDCl}_{3}\right): \delta 9.23(\mathrm{~d}, J=8.7 \mathrm{~Hz}, 1 \mathrm{H}), 8.25(\mathrm{dd}, J=7.3 \mathrm{~Hz}, 1.2 \mathrm{~Hz}, 1 \mathrm{H})$, $8.00(\mathrm{~d}, J=8.3 \mathrm{~Hz}, 1 \mathrm{H}), 7.94(\mathrm{~d}, J=7.8,1 \mathrm{H}), 7.70-7.65(\mathrm{~m}, 1 \mathrm{H}), 7.62-7.56(\mathrm{~m}, 2 \mathrm{H})$, $7.41\left(\mathrm{~s}, 1 \mathrm{H}\right.$, oxazole $\mathrm{CH}$ ), $5.77\left(\mathrm{t}, J=10.1 \mathrm{~Hz}, 1 \mathrm{H}, \mathrm{H}^{2}\right.$ ), $5.57(\mathrm{dd}, J=3.5 \mathrm{~Hz}, 0.9 \mathrm{~Hz}, 1 \mathrm{H}$, $\mathbf{H}^{4}$ ), 5.24 (dd, J=10.4 Hz, 3.4 Hz, 1H, H3), 4.73 (d, J=10.1 Hz, 1H, $\mathbf{H}^{1}$ ), 4.23-4.12 (m, 3H), $2.26(\mathrm{~s}, 3 \mathrm{H}), 2.09(\mathrm{~s}, 3 \mathrm{H}), 2.06(\mathrm{~s}, 3 \mathrm{H}), 1.96(\mathrm{~s}, 3 \mathrm{H})$

${ }^{13} \mathrm{C}$ NMR $\left(100 \mathrm{MHz}, \mathrm{CDCl}_{3}\right): \delta 134.16,134.01,131.65,131.19,129.13,129,02$, $128.59,128.41,127.89,127.67,126.38,126.00,124.99,74.77,72.23,72.07$, $67.46,66.94,61.64,20.80,20.75,20.63$

$[\alpha]^{20}{ }_{D}=-3^{\circ}(c=0.30249$ in chloroform $)$.

HRMS: M+H; 526.1719 found, 526.1713 calculated

\section{2-Naphth-2-yl-5-(2,3,4,6-tetra-O-acetyl-1-deoxy- $\beta$-D-galactopyranosyl)oxa- zole 10c}

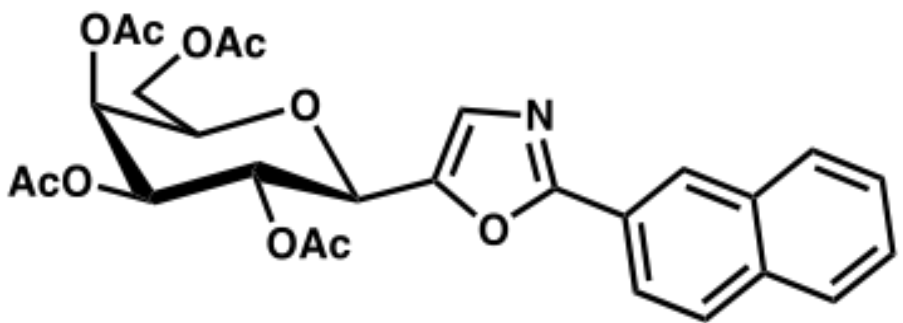

Compound 8 (100 mg, $0.281 \mathrm{mmol}$ ), 8-methylquinoline $\mathrm{N}$-oxide (67 mg, 0.421 mmol), [bis(trifluoromethanesulfonyl)imidate](triphenylphosphine)gold(I) 2:1 toluene adduct (22 $\mathrm{mg}, 0.028 \mathrm{mmol}$ ) and 2-cyanonaphthalene (150 $\mathrm{mg}, 0.980$ mmol) were mixed, and heated to $75^{\circ} \mathrm{C}$ where the mixture melted. The reaction was left under nitrogen for 48 hours, then diluted with ethyl acetate $(25 \mathrm{~mL})$ and washed with brine $(40 \mathrm{~mL})$. The brine was extracted two times with ethyl acetate $(20 \mathrm{~mL})$, the organic phases were pooled, dried with anhydrous sodium sulfate and evaporated. Column chromatography (1.5:1 heptane/ethyl acetate) gave 10c (35 mg, 25\%).

${ }^{1} \mathrm{H}$ NMR (400 MHz, CDCl $): \delta 8.59(\mathrm{~d}, J=1.3 \mathrm{~Hz}, 1 \mathrm{H}), 8.15(\mathrm{dd}, J=8.7 \mathrm{~Hz}, 1.6 \mathrm{~Hz}, 1 \mathrm{H})$, 8.00-7.87 (m, 3H), 7.59-7.55 (m, 2H), $7.32(\mathrm{~s}, 1 \mathrm{H}$, oxazole $\mathrm{CH}), 5.73(\mathrm{t}, J=10.1 \mathrm{~Hz}$, $1 \mathrm{H}, \mathbf{H}^{2}$ ), 5.57 (dd, J=3.5 Hz, $0.9 \mathrm{~Hz}, 1 \mathrm{H}, \mathbf{H}^{4}$ ), 5.22 (dd, J=10.4 Hz, 3.4 Hz, 1H, H ), $4.71\left(\mathrm{~d}, J=10.1 \mathrm{~Hz}, 1 \mathrm{H}, \mathbf{H}^{1}\right.$ ) , 4.23-4.11 (m, 3H), 2.28 (s, 3H), 2.09 (s, 3H), 2.05 (s, $3 \mathrm{H}), 1.95(\mathrm{~s}, 3 \mathrm{H})$ ${ }^{13} \mathrm{C}$ NMR $\left(100 \mathrm{MHz}^{\mathrm{CDCl}} 3\right): \delta 170.47,170.37,170.17,169.16,146.61,128.82$, 128.71, 128.19, 127.89, 127.47, 126.82, 126.76, 123.35, 74.74, 72.25, 72.09, $67.43,66.85,61.59,20.82,20.75,20.62$ $[\alpha]^{20}{ }_{D}=-8^{\circ}(c=0.20941$ in chloroform $)$. HRMS: $\mathrm{M}+\mathrm{H}$; 526.1712 found, 526.1713 calculated. 


\section{2-(4-Chlorophenyl)-5-(2,3,4,6-tetra- $O$-acetyl-1-deoxy- $\beta$-D-galactopyra- nosyl)oxazole 10d}

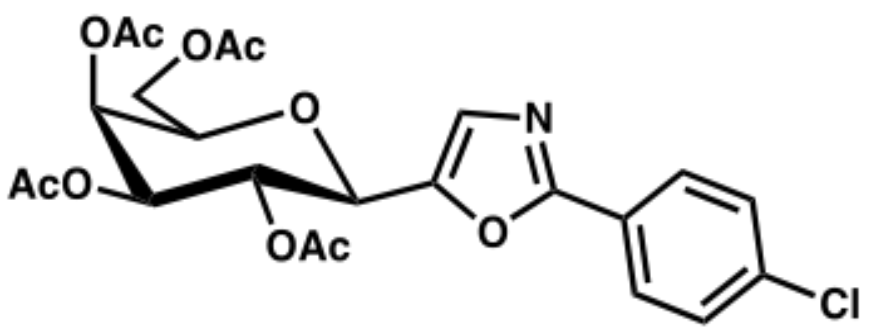

Compound 8 (50 mg, 0.140 mmol), 8-methylquinoline N-oxide (49 mg, 0.182 mmol), [bis(trifluoromethanesulfonyl)imidate](triphenylphosphine)gold(I) 2:1 toluene adduct ( $11 \mathrm{mg}, 0.007 \mathrm{mmol}$ ) and of 4-chlorobenzonitrile (116 mg, 0.840 mmol) was sealed in a tube under nitrogen, heated to $75^{\circ} \mathrm{C}$ under nitrogen, and left for 3 days. The reaction was diluted with ethyl acetate $(20 \mathrm{~mL})$, poured into brine $(20 \mathrm{~mL})$ and extracted two times with ethyl acetate $(20 \mathrm{~mL})$. The organic phases were pooled, dried with anhydrous sodium sulfate and evaporated. Column chromatography (1:1 heptane/ethyl acetate) gave yellow powdered 10d ( 9 mg, 19\%).

${ }^{1} \mathrm{H} \mathrm{NMR}\left(400 \mathrm{MHz}, \mathrm{CDCl}_{3}\right): \delta 8.04-7.98(\mathrm{~m}, 2 \mathrm{H}), 7.49-7.44(\mathrm{~m}, 2 \mathrm{H}), 7.28(\mathrm{~s}, 1 \mathrm{H}, \mathrm{ox}-$ azole $\mathrm{CH}$ ), $5.67\left(\mathrm{t}, J=10.1 \mathrm{~Hz}, 1 \mathrm{H}, \mathbf{H}^{2}\right) .5 .55\left(\mathrm{dd}, J=3.5 \mathrm{~Hz}, 1.0 \mathrm{~Hz}, 1 \mathrm{H}, \mathbf{H}^{4}\right), 5.19$ (dd, $J=10.1 \mathrm{~Hz}, 3.4 \mathrm{~Hz}, 1 \mathrm{H}, \mathbf{H}^{3}$ ), $4.64\left(\mathrm{~d}, J=10.0 \mathrm{~Hz}, 1 \mathrm{H}, \mathbf{H}^{1}\right), 4.21-4.08(\mathrm{~m}, 3 \mathrm{H}), 2.25$ (s, $3 \mathrm{H}), 2.08(\mathrm{~s}, 3 \mathrm{H}), 2.03(\mathrm{~s}, 3 \mathrm{H}), 1.93(\mathrm{~s}, 3 \mathrm{H})$

${ }^{13} \mathrm{C}$ NMR $\left(100 \mathrm{MHz}, \mathrm{CDCl}_{3}\right): \delta 170.45,170.31,170.13,169.12,161.76,146.63$, $136.93,129.16,128.22,127.91,125.56,74.74,72.11,71.99,67.38,66.77,61.56$, $20.79,20.73,20.62,20.59$

HRMS: $\mathrm{M}+\mathrm{H} ; 510.1167$ found, 510.1167 calculated. $[\alpha]^{20}{ }_{D}=-11^{\circ}(c=0.23056$ in chloroform $)$.

\section{2-(3-Chlorophenyl)-5-(2,3,4,6-tetra- $O$-acetyl-1-deoxy- $\beta$-D-galactopyra- nosyl)oxazole 10e}

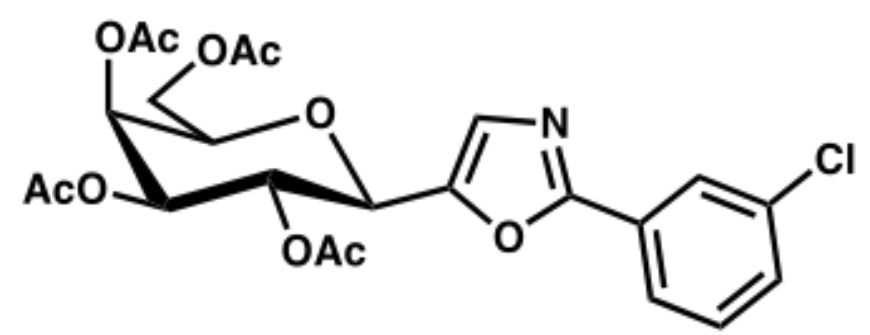

Compound 8 (50 mg, $0.140 \mathrm{mmol}$ ), 8-methylquinoline $\mathrm{N}$-oxide (49 mg, 0.182 mmol), [bis(trifluoromethanesulfonyl)imidate](triphenylphosphine)gold(I) 2:1 toluene adduct (11 mg, $0.007 \mathrm{mmol}$ ) and 3-chlorobenzonitrile (116 mg, 0.840 mmol) were sealed in a tube under nitrogen, heated to $50^{\circ} \mathrm{C}$ under nitrogen, and left for 3 days. The reaction was diluted with ethyl acetate $(20 \mathrm{~mL})$, poured into brine $(20 \mathrm{~mL})$ and extracted two times with ethyl acetate $(20 \mathrm{~mL})$. The organic phases were pooled, dried with anhydrous sodium sulfate and evaporated. Column chromatography (1:1 heptane/ethyl acetate) gave yellow powdered 10e (8 mg, 11\%).

${ }^{1} \mathrm{H}$ NMR (400 MHz, CDCl $): \delta 8.07(\mathrm{t}, J=1.7 \mathrm{~Hz}, 1 \mathrm{H}), 7.96(\mathrm{dt}, \mathrm{J} 1=7.3 \mathrm{~Hz}, 1.7 \mathrm{~Hz}$, 1H), 7.48-7.40 (m, 2H), 7.27 (s, 1H, oxazole CH), $5.67\left(\mathrm{t}, J=10.0 \mathrm{~Hz}, 1 \mathrm{H}, \mathbf{H}^{2}\right) .5 .55$ 
(dd, J=3.6 Hz, 1.2 Hz, 1H, H H) $^{4} .20$ (dd, J=10.0 Hz, $3.6 \mathrm{~Hz}, 1 \mathrm{H}, \mathbf{H}^{3}$ ), 4.65 (d, J=10.0 $\mathrm{Hz}, 1 \mathrm{H}, \mathrm{H}^{1}$ ), 4.21-4.09 (m, 3H), 2.26 (s, 3H), 2.09 (s, 3H), $2.04(\mathrm{~s}, 3 \mathrm{H}), 1.94(\mathrm{~s}, 3 \mathrm{H})$ ${ }^{13} \mathrm{C}$ NMR $\left(100 \mathrm{MHz}, \mathrm{CDCl}_{3}\right): \delta 170.45,170.32,170.12,169.11,134.94,130.76$, 130.17, 128.69, 128.19, 126.66, 124.70, 74.78, 72.14, 71.97, 67.38, 66.82, 61.57, 20.80, 20.73, 20.62, 20.58

HRMS: $\mathrm{M}+\mathrm{H}$; 510.1177 found, 510.1167 calculated.

$[\alpha]^{20}{ }_{D}=-8^{\circ}(c=0.27875$ in chloroform $)$.

\section{2-(4-Methylphenyl)-5-(2,3,4,6-tetra- $O$-acetyl-1-deoxy- $\beta$-D-galactopyra- nosyl)oxazole $10 \mathrm{f}$}

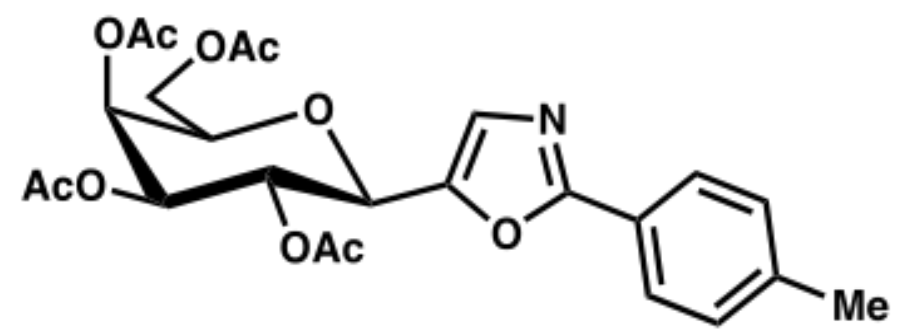

Compound 8 (50 mg, 0.140 mmol), 8-methylquinoline N-oxide (49 mg, 0.182 mmol), [bis(trifluoromethanesulfonyl)imidate](triphenylphosphine)gold(I) 2:1 toluene adduct ( $11 \mathrm{mg}, 0.007 \mathrm{mmol}$ ) and p-tolunitrile ( $99 \mathrm{mg}, 0.840 \mathrm{mmol}$ ) were sealed in a tube under nitrogen, heated to $75^{\circ} \mathrm{C}$ under nitrogen, and left for 3 days. The reaction was diluted with ethyl acetate $(20 \mathrm{~mL})$, poured into brine $(20$ $\mathrm{mL})$ and extracted two times with ethyl acetate $(20 \mathrm{~mL})$. The organic phases were pooled, dried with anhydrous sodium sulfate and evaporated. Column chromatography (1:1 heptane/ethyl acetate) gave yellow powdered $10 f(3 \mathrm{mg}$, $4 \%)$.

${ }^{1} \mathrm{H}$ NMR (400 MHz, $\left.\mathrm{CDCl}_{3}\right): \delta$ 7.98-7.94 (m, 2H), 7.31-7.27 (m, 3H), 7.79-7.74 (m, $1 \mathrm{H}), 5.68\left(\mathrm{t}, J=10.1 \mathrm{~Hz}, 1 \mathrm{H}, \mathbf{H}^{2}\right.$ ), $5.54\left(\mathrm{dd}, J=3.4 \mathrm{~Hz}, 1.0 \mathrm{~Hz}, 1 \mathrm{H}, \mathbf{H}^{4}\right), 5.19$ (dd, $J=10.2 \mathrm{~Hz}, 3.8 \mathrm{~Hz}, 1 \mathrm{H}, \mathbf{H}^{3}$ ), 4.65 (d, J=9.6 Hz, 1H, H' $\left.{ }^{1}\right), 4.23-4.08(\mathrm{~m}, 3 \mathrm{H}), 2.43$, (s, 3H, phenyl methyl H), $2.25(\mathrm{~s}, 3 \mathrm{H}), 2.08(\mathrm{~s}, 3 \mathrm{H}), 2.04(\mathrm{~s}, 3 \mathrm{H}), 1.94(\mathrm{~s}, 3 \mathrm{H})$ ${ }^{13} \mathrm{C}$ NMR $\left(100 \mathrm{MHz} \mathrm{CClD}_{3}\right): \delta 170.47,170.36,170.17,169.10,141.13,130.20$, $129.52,129.20,127.92,126.58,124.37,118.10,74.67,72.20,72.08,67.43,66.81$, $61.59,21.57,20.80,20.74,20.63,20.60$

HRMS: $\mathrm{M}+\mathrm{H} ; 490.1707$ found, 490.1713 calculated. $[\alpha]^{20} D^{2}=-13^{\circ}(c=0.18849$ in chloroform $)$. 


\section{2-(3-Methylphenyl)-5-(2,3,4,6-tetra- $O$-acetyl-1-deoxy- $\beta$-D-galactopyra- nosyl)oxazole $10 \mathrm{~g}$}

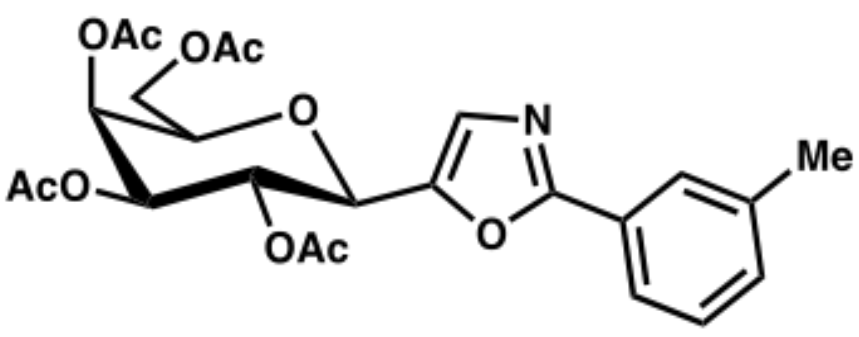

Compound 8 (50 mg, $0.140 \mathrm{mmol}$ ), 8-methylquinoline N-oxide (49 mg, 0.182 mmol), [bis(trifluoromethanesulfonyl)imidate](triphenylphosphine)gold(I) 2:1 toluene adduct ( $11 \mathrm{mg}, 0.007 \mathrm{mmol}$ ) and m-tolunitrile ( $99 \mathrm{mg}, 0.840 \mathrm{mmol}$ ) were sealed in a tube under nitrogen, heated to $75^{\circ} \mathrm{C}$ under nitrogen, and left for 3 days. The reaction was diluted with ethyl acetate $(20 \mathrm{~mL})$, poured into brine $(20 \mathrm{~mL})$ and extracted two times with ethyl acetate $(20 \mathrm{~mL})$. The organic phases were pooled, dried with anhydrous sodium sulfate and evaporated. Column chromatography (1:1 heptane/ethyl acetate) gave yellow powdered $\mathbf{1 0 g}$ (22 mg, $48 \%)$.

${ }^{1} \mathrm{H}$ NMR (400 MHz, $\mathrm{CDCl}_{3}$ ): $\delta$ 7.94-7.84 (m ,2H), $7.37(\mathrm{t}, J=7.6 \mathrm{~Hz}, 1 \mathrm{H}), 7.31-7.23$ $(\mathrm{m}, 2 \mathrm{H}), 5.67\left(\mathrm{t}, J=10.2 \mathrm{~Hz}, 1 \mathrm{H}, \mathbf{H}^{2}\right), 5.54\left(\mathrm{dd}, J=3.5 \mathrm{~Hz}, 0.9 \mathrm{~Hz}, 1 \mathrm{H}, \mathbf{H}^{4}\right.$ ), 5.19 (dd, $J=9.9 \mathrm{~Hz}, 3.4 \mathrm{~Hz}, 1 \mathrm{H}, \mathbf{H}^{3}$ ), $4.66\left(\mathrm{~d}, J=10.2 \mathrm{~Hz}, 1 \mathrm{H}, \mathbf{H}^{1}\right), 4.22-4.08(\mathrm{~m}, 3 \mathrm{H}), 2.44$, (s, $3 \mathrm{H}$, phenyl methyl $\mathrm{H}), 2.25(\mathrm{~s}, 3 \mathrm{H}), 2.08(\mathrm{~s}, 3 \mathrm{H}), 2.03(\mathrm{~s}, 3 \mathrm{H}), 1.93(\mathrm{~s}, 3 \mathrm{H})$ ${ }^{13} \mathrm{C}$ NMR $\left(100 \mathrm{MHz}, \mathrm{CDCl}_{3}\right): \delta 170.46,170.35,170.16,169.11,146.30,138.60$, 131.58, 128.73, 127.93, 127.17, 126.94, 123.76, 74.69, 72.21, 72.07, 67.42, 66.84, 61.58, 21.36, 20.80, 20.74, 20.63, 20.59

HRMS: M+H; 490.1707 found, 490.1713 calculated $[\alpha]^{20}{ }_{D}=-6^{\circ}(c=0.55960$ in chloroform $)$.

\section{2-(4-Fluorophenyl)-5-(2,3,4,6-tetra- $O$-acetyl-1-deoxy- $\beta$-D-galactopyra- nosyl)oxazole $10 \mathrm{~h}$}

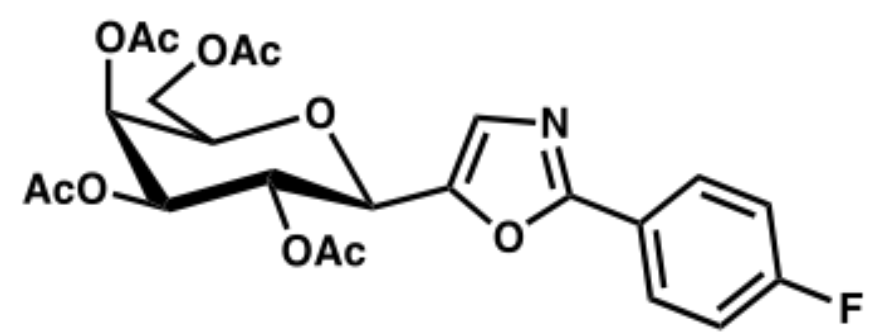

Compound 8 (50 mg, $0.140 \mathrm{mmol}$ ), 8-methylquinoline N-oxide (49 mg, 0.182 mmol), [bis(trifluoromethanesulfonyl)imidate](triphenylphosphine)gold(I) 2:1 toluene adduct (11 mg, $0.007 \mathrm{mmol}$ ) and 4-fluorobenzonitrile (102 $\mathrm{mg}, 0.840$ mmol) were sealed in a tube under nitrogen, heated to $75^{\circ} \mathrm{C}$ under nitrogen, and left for 3 days. The reaction was diluted with ethyl acetate $(20 \mathrm{~mL})$, poured into brine $(20 \mathrm{~mL})$ and extracted two times with ethyl acetate $(20 \mathrm{~mL})$. The organic phases were pooled, dried with anhydrous sodium sulfate and evaporated. Column chromatography (1:1 heptane/ethyl acetate) gave yellow powdered $\mathbf{1 0 h}$ (8 mg, 11\%). 
${ }^{1} \mathrm{H}$ NMR (400 MHz, $\left.\mathrm{CDCl}_{3}\right): \delta 8.10-8.04(\mathrm{~m}, 2 \mathrm{H}), 7.24(\mathrm{~s}, 1 \mathrm{H}$, oxazole $\mathrm{CH}), 7.21-$ $7.14(\mathrm{~m}, 2 \mathrm{H}), 5.67\left(\mathrm{t}, J=10.8 \mathrm{~Hz}, 1 \mathrm{H}, \mathbf{H}^{2}\right), 5.55\left(\mathrm{dd}, J=3.5 \mathrm{~Hz}, 1.0 \mathrm{~Hz}, 1 \mathrm{H}, \mathbf{H}^{4}\right), 5.19$ (dd, J=9.8 Hz, 3.4 Hz, 1H, H ${ }^{3}$ ), $4.64\left(\mathrm{~d}, J=10.8 \mathrm{~Hz}, 1 \mathrm{H}, \mathbf{H}^{1}\right.$ ), 4.21-4.06 (m, 3H), 2.25 $(\mathrm{s}, 3 \mathrm{H}), 2.08(\mathrm{~s}, 3 \mathrm{H}), 2.04(\mathrm{~s}, 3 \mathrm{H}), 1.94(\mathrm{~s}, 3 \mathrm{H})$ ${ }^{13} \mathrm{C}$ NMR $\left(100 \mathrm{MHz}, \mathrm{CDCl}_{3}\right): \delta 170.45,170.31,170.14,169.12,146.42,128.86$, $128.77,128.14,116.15,115.93,74.72,72.13,72.01,67.39,66.77,61.56,20.78$, $20.74,20.62,20.59$

HRMS: $\mathrm{M}+\mathrm{H} ; 494.1463$ found, 494.1462 calculated. $[\alpha]^{20}{ }_{D}=-6^{\circ}(c=0.20000$ in chloroform $)$.

\section{2-(3-Fluorophenyl)-5-(2,3,4,6-tetra- $O$-acetyl-1-deoxy- $\beta$-D-galactopyra- nosyl)oxazole 10i}

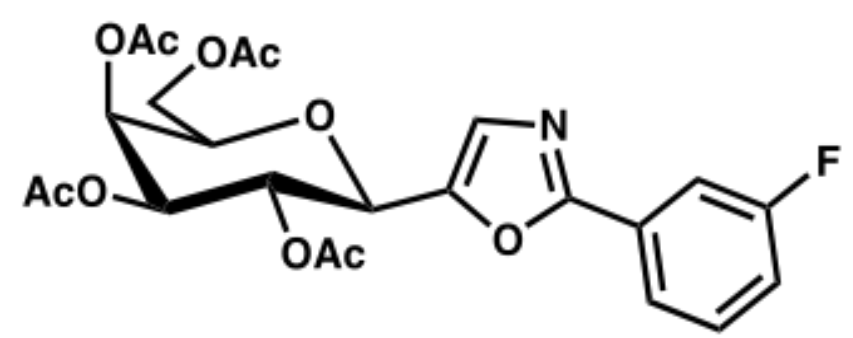

Compound 8 ( $50 \mathrm{mg}, 0.140 \mathrm{mmol}$ ), 8-methylquinoline $\mathrm{N}$-oxide (49 mg, 0.182 mmol), [bis(trifluoromethanesulfonyl)imidate](triphenylphosphine)gold(I) 2:1 toluene adduct (11 mg, $0.007 \mathrm{mmol}$ ) and 3-fluorobenzonitrile (101 $\mathrm{mg}, 0.840$ mmol) were sealed in a tube under nitrogen, heated to $75^{\circ} \mathrm{C}$ under nitrogen, and left for 3 days. The reaction was diluted with ethyl acetate $(20 \mathrm{~mL})$, poured into brine $(20 \mathrm{~mL})$ and extracted two times with ethyl acetate $(20 \mathrm{~mL})$. The organic phases were pooled, dried with anhydrous sodium sulfate and evaporated. Column chromatography (1:1 heptane/ethyl acetate) gave 10i (14 mg, 31\%) as a yellow viscous oil.

${ }^{1} \mathrm{H}$ NMR $\left(400 \mathrm{MHz}, \mathrm{CDCl}_{3}\right): \delta 7.86(\mathrm{~d}, J=7.8 \mathrm{~Hz}, 1 \mathrm{H}), 7.79-7.74(\mathrm{~m}, 1 \mathrm{H}), 7.50-7.42$ $(\mathrm{m}, 1 \mathrm{H}), 7.28(\mathrm{~s}, 1 \mathrm{H}$, oxazole $\mathrm{CH}), 7.22-7.15(\mathrm{~m}, 1 \mathrm{H}), 5.66\left(\mathrm{t}, J=9.9 \mathrm{~Hz}, 1 \mathrm{H}, \mathbf{H}^{2}\right.$ ), 5.55 (dd, J=3.5 Hz, $1.0 \mathrm{~Hz}, 1 \mathrm{H}, \mathbf{H}^{4}$ ), 5.19 (dd, J=10.2 Hz, $3.2 \mathrm{~Hz}, 1 \mathrm{H}, \mathbf{H}^{3}$ ), 4.65 (d, $\left.J=10.2 \mathrm{~Hz}, 1 \mathrm{H}, \mathrm{H}^{1}\right), 4.24-4.09(\mathrm{~m}, 3 \mathrm{H}), 2.25(\mathrm{~s}, 3 \mathrm{H}), 2.08(\mathrm{~s}, 3 \mathrm{H}), 2.04(\mathrm{~s}, 3 \mathrm{H}), 1.94$ $(\mathrm{s}, 3 \mathrm{H})$

${ }^{13} \mathrm{C}$ NMR $\left(100 \mathrm{MHz}, \mathrm{CDCl}_{3}\right): \delta 170.46,170.33,170.14,169.12,161.65,130.62$, 130.54, 129.02, 128.17, 122.32, 117.87, 117.66, 113.75, 113.50, 74.76, 72.10, 71.97, 67.39, 66.81, 61.56, 20.79, 20.73, 20.62, 20.59

HRMS: $\mathrm{M}+\mathrm{H}$; 494.1470 found, 494.1482 calculated. $[\alpha]^{20} \mathrm{D}=-4^{\circ}(c=0.41667$ in chloroform $)$. 


\section{NMR spectra}

1a: 1-phenyl-4-(1-deoxy- $\beta$-D-galactopyranosyl)triazole

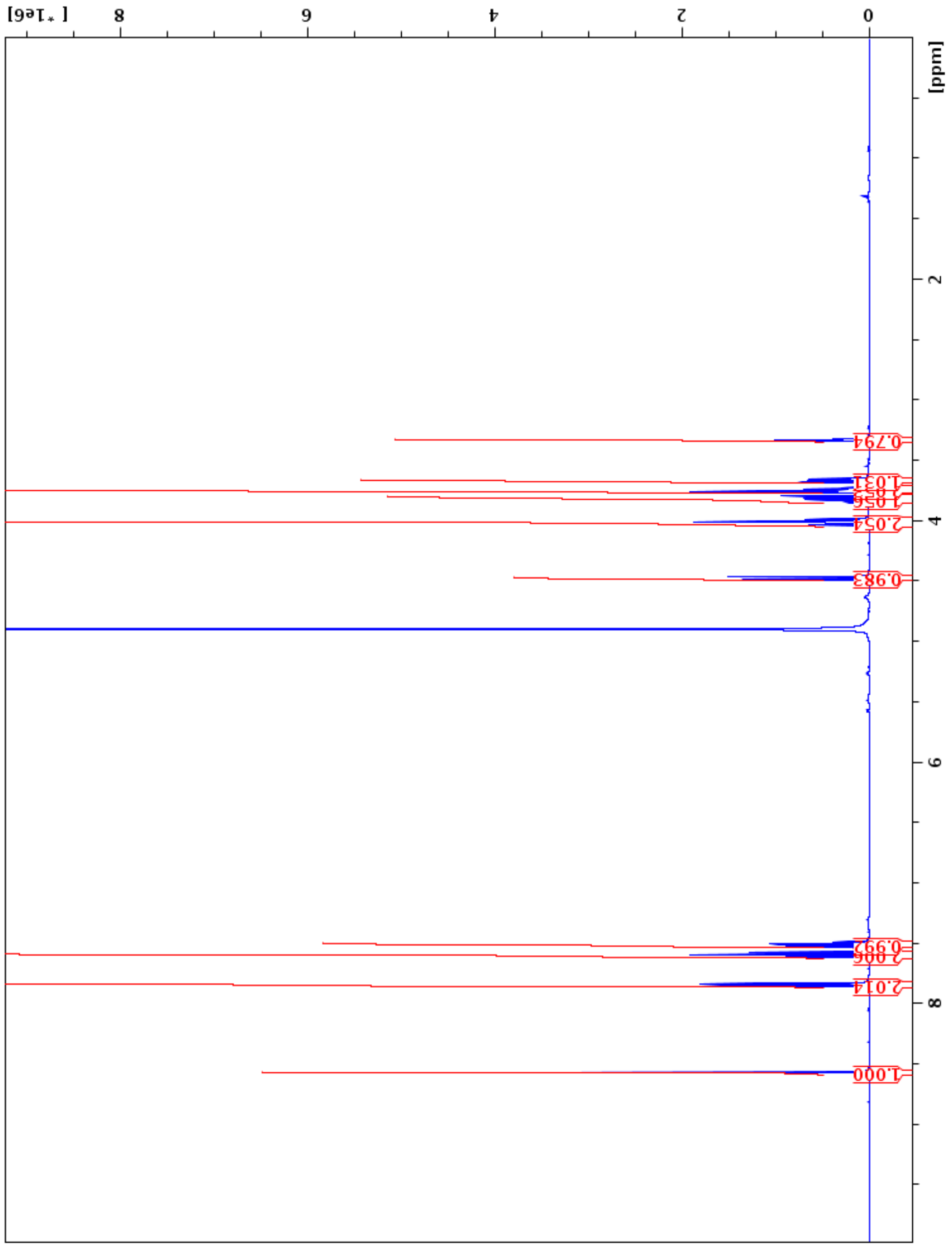


1a: 1-phenyl-4-(1-deoxy- $\beta$-D-galactopyranosyl)triazole

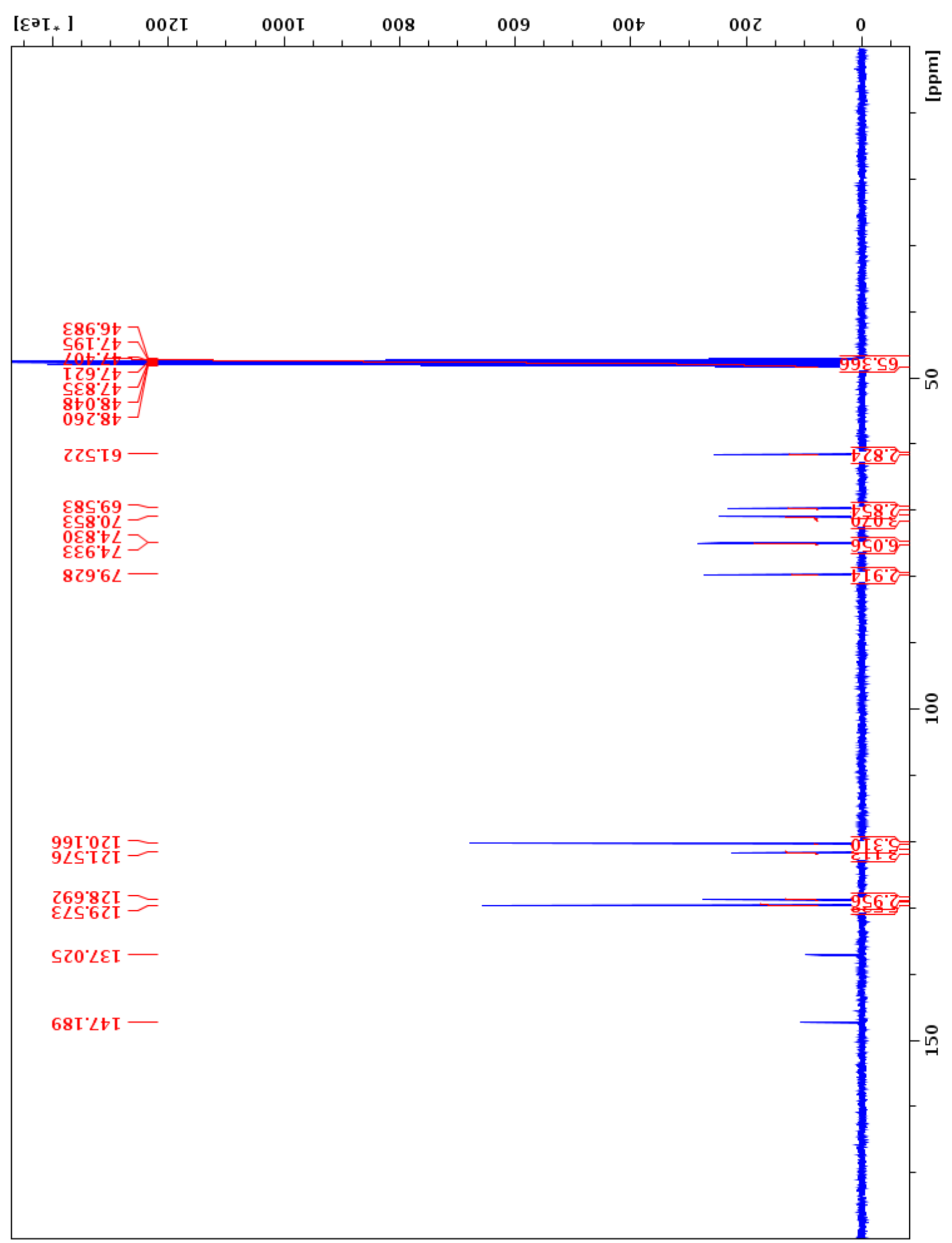


1b: 1-naphth-1-yl-4-(1-deoxy- $\beta$-D-galactopyranosyl)oxazole

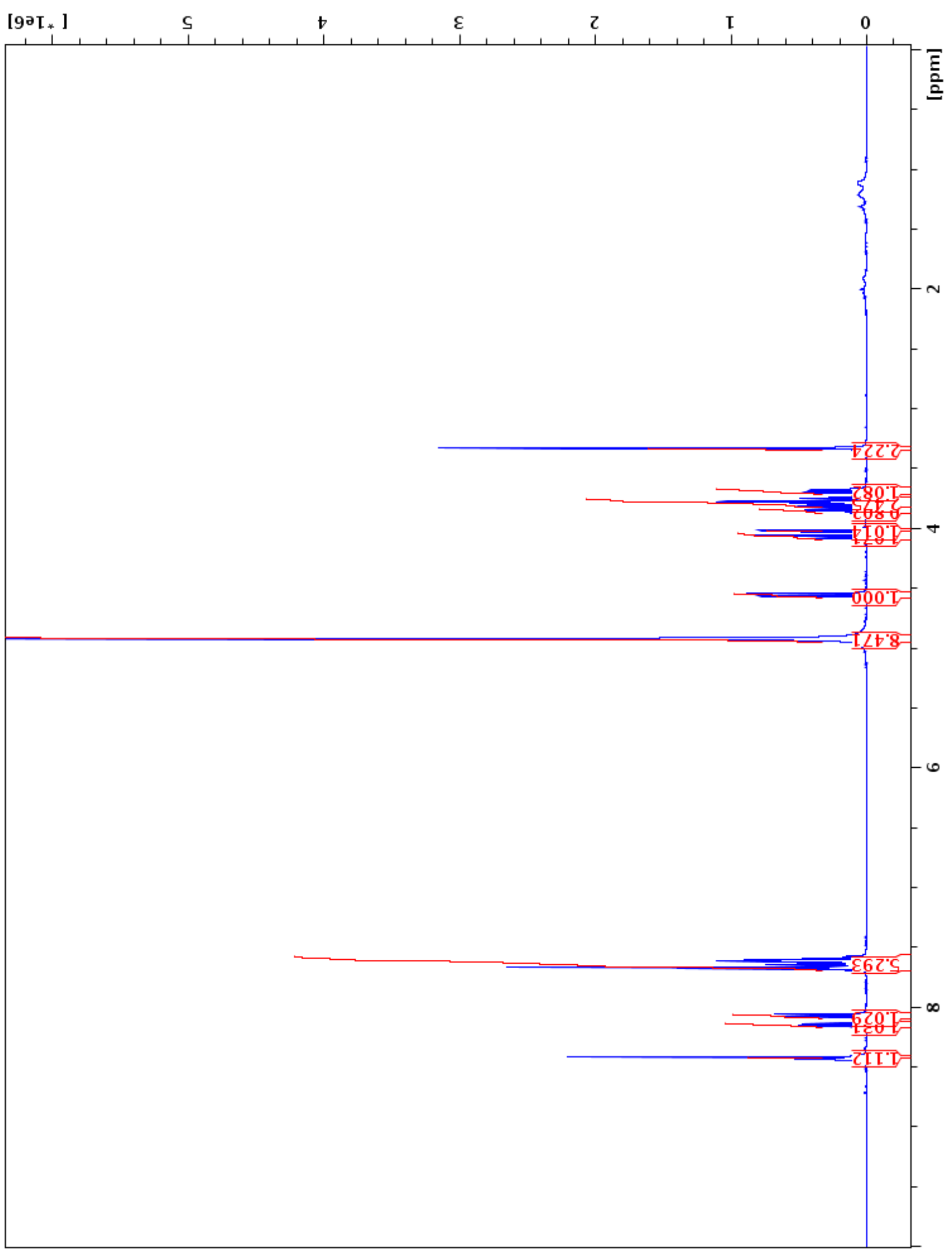


1b: 1-naphth-1-yl-4-(1-deoxy- $\beta$-D-galactopyranosyl)triazole

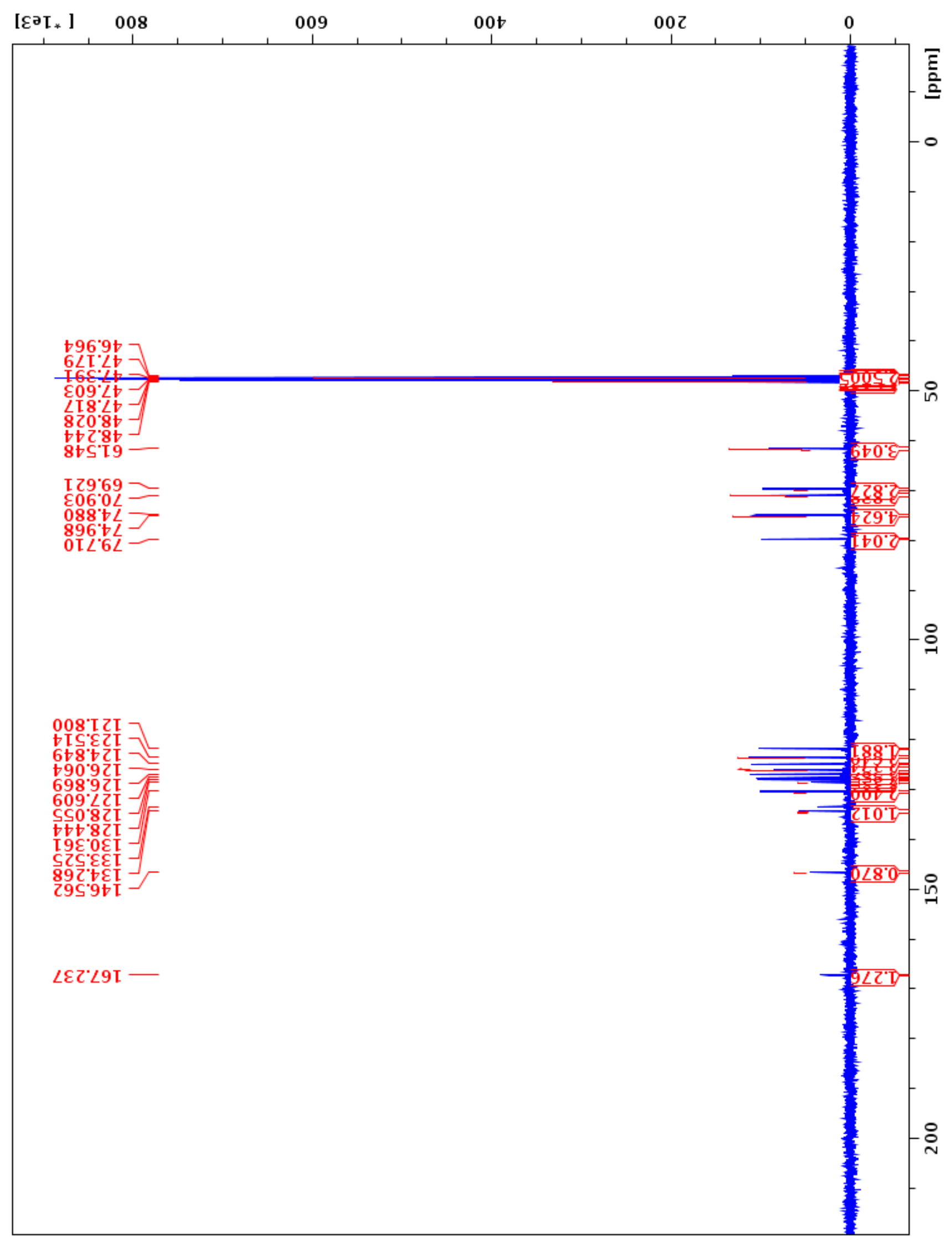


1c: 1-naphth-2-yl-4-(1-deoxy- $\beta$-D-galactopyranosyl)triazole

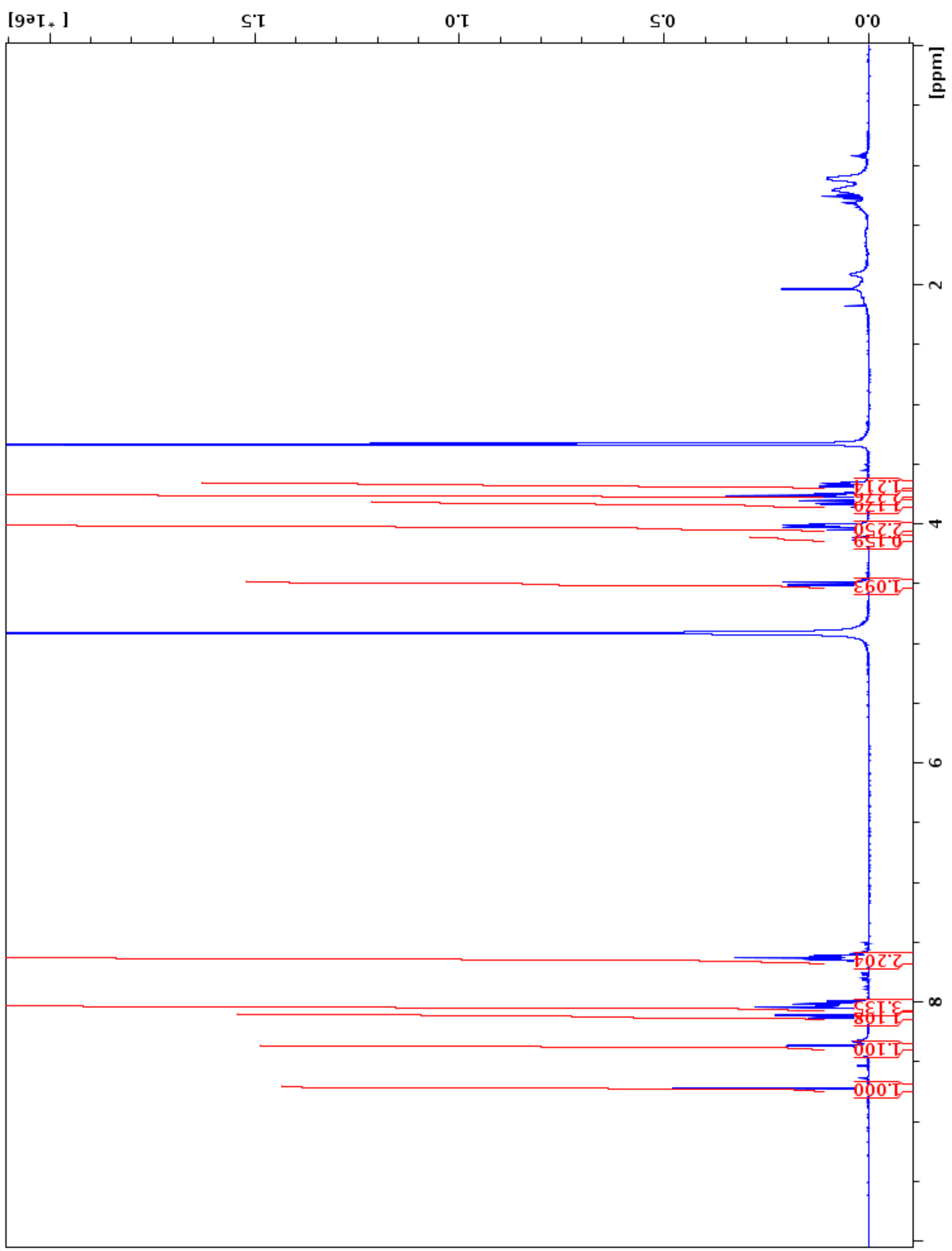


1c: 1 -(naphth-2-yl)-4-(1-deoxy- $\beta$-D-galactopyranosyl)triazole

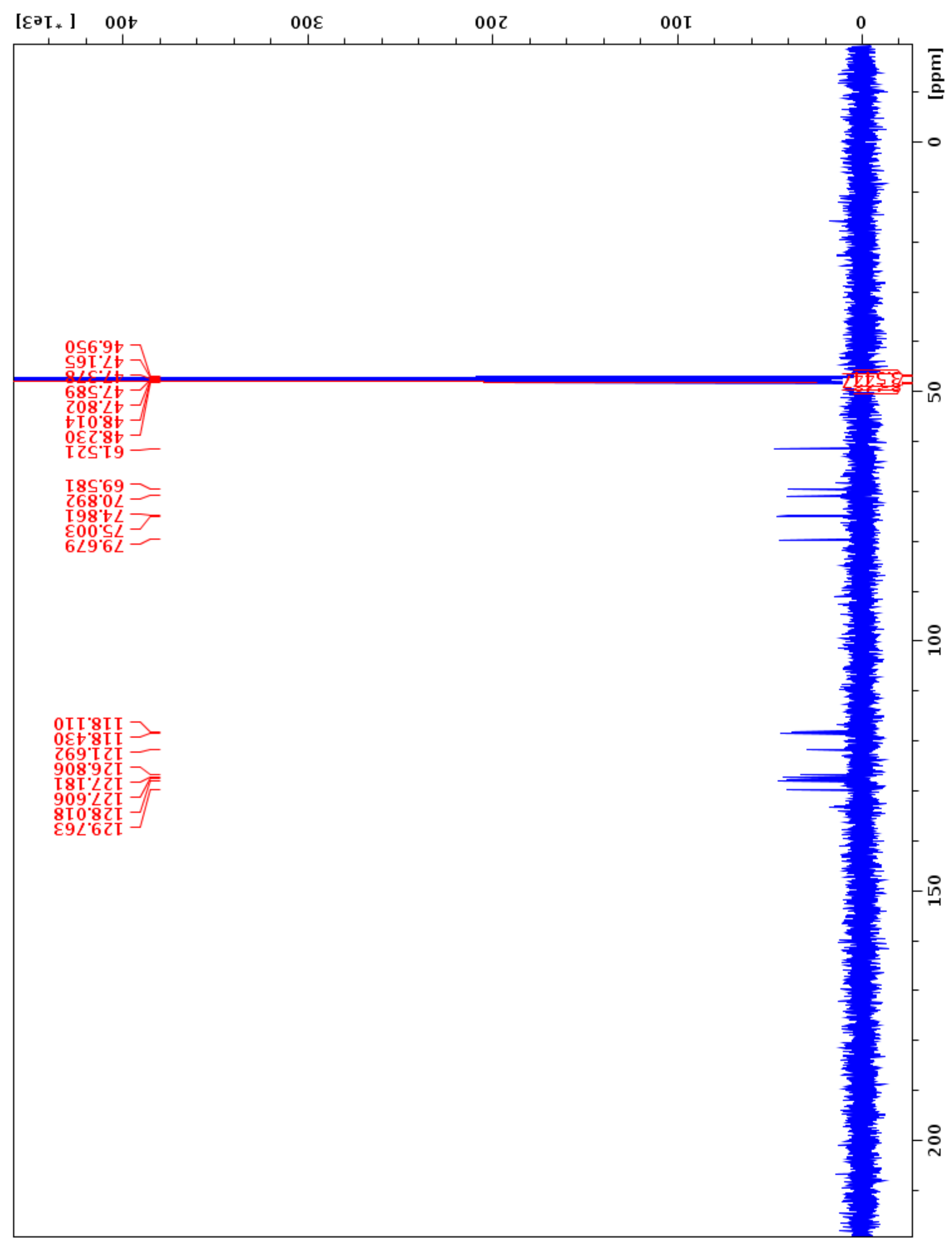


1d: 1 -(4-chlorophenyl)-4-(1-deoxy- $\beta$-D-galactopyranosyl)triazole

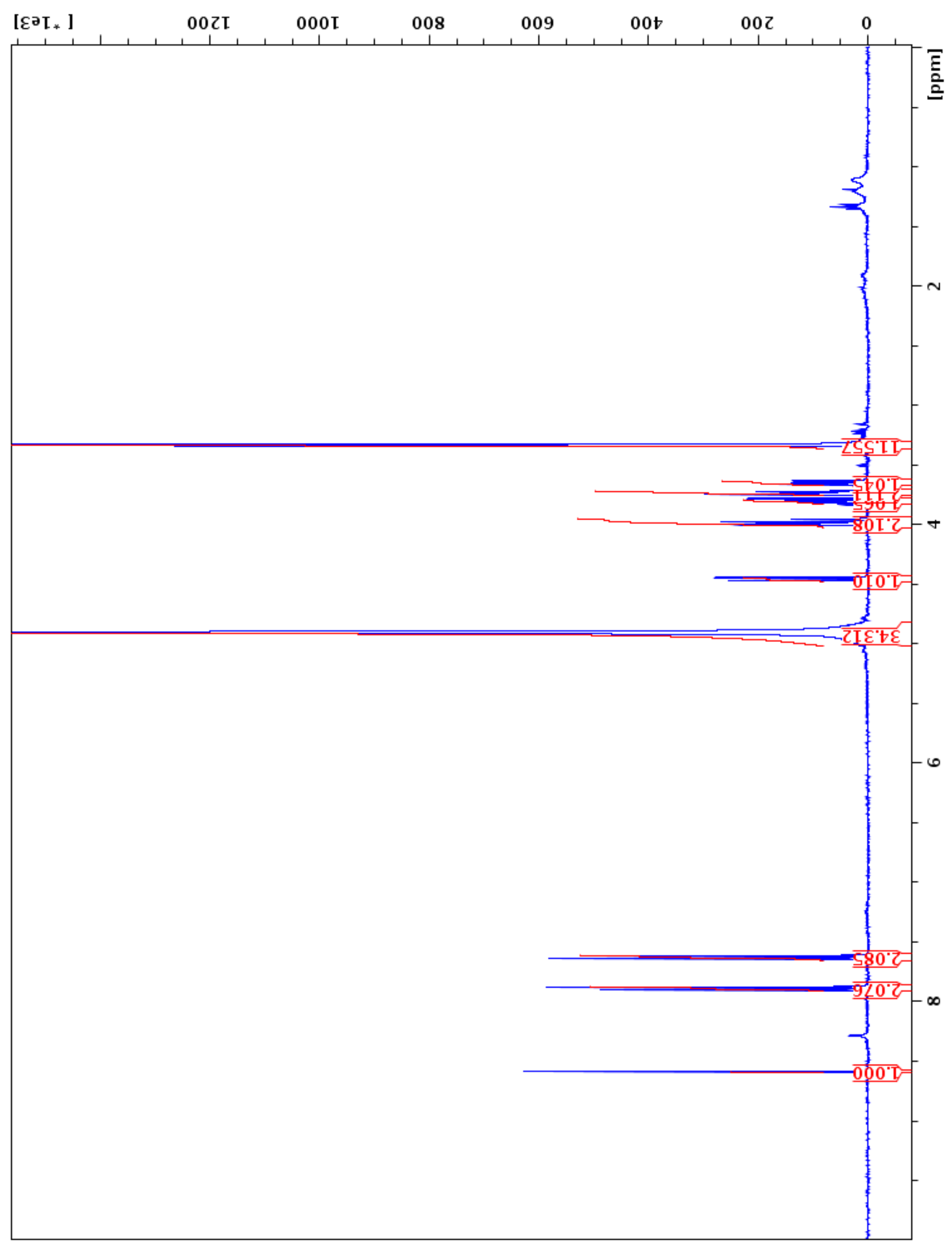


1d: 1 -(4-chlorophenyl)-4-(1-deoxy- $\beta$-D-galactopyranosyl)triazole

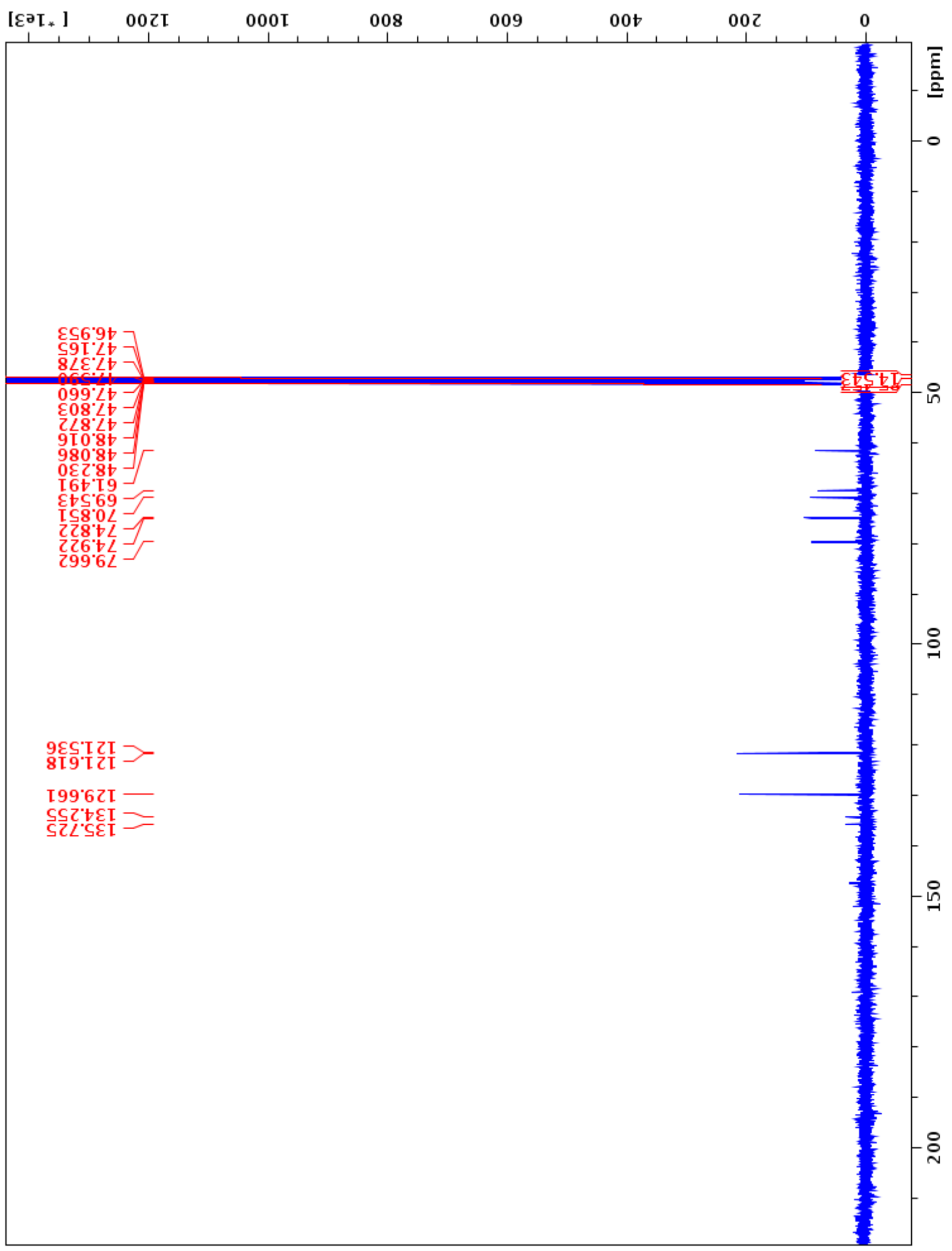


1e: 1 -(3-chlorophenyl)-4-(1-deoxy- $\beta$-D-galactopyranosyl)triazole

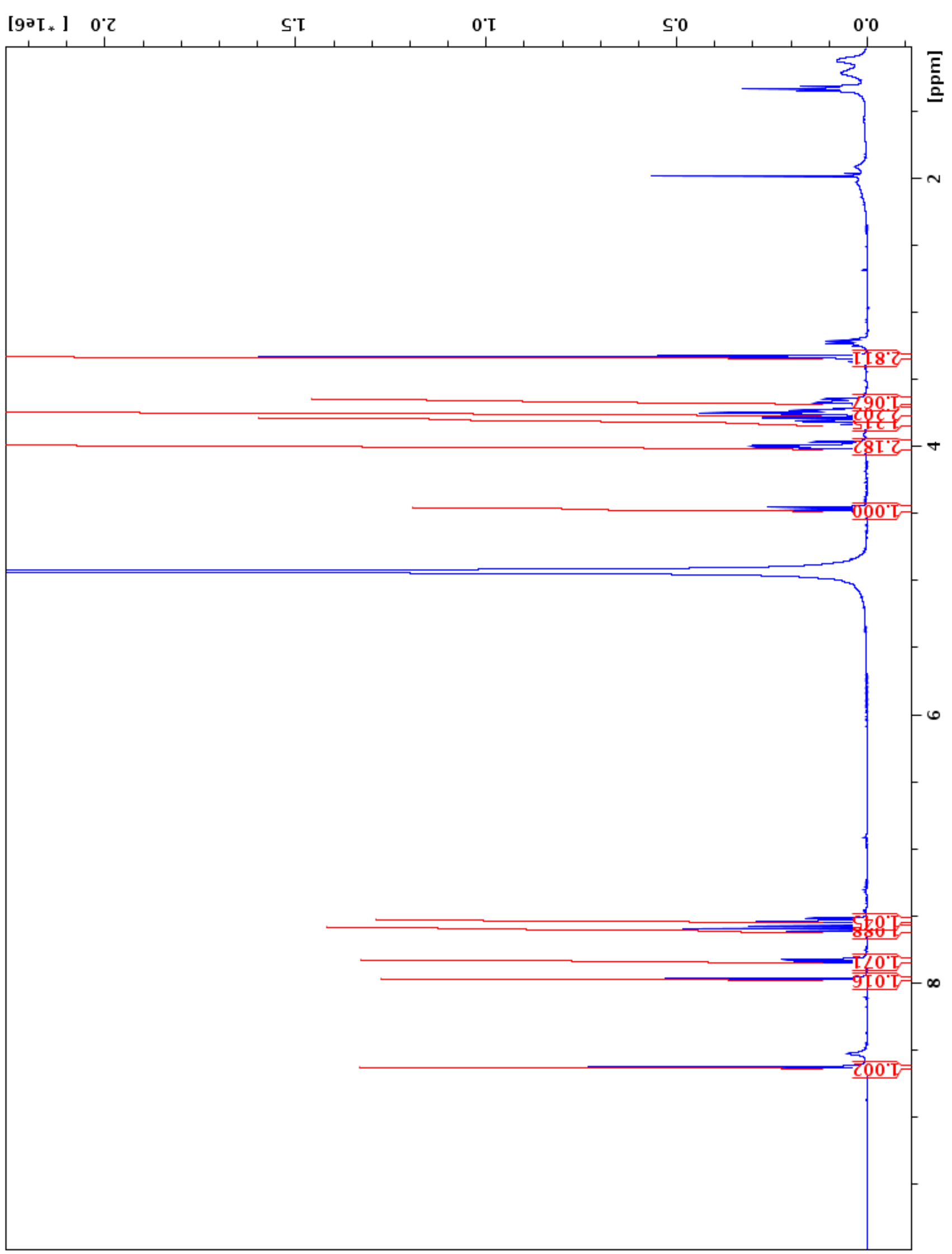


1e: 1-(3-chlorophenyl)-4-(1-deoxy- $\beta$-D-galactopyranosyl)triazole

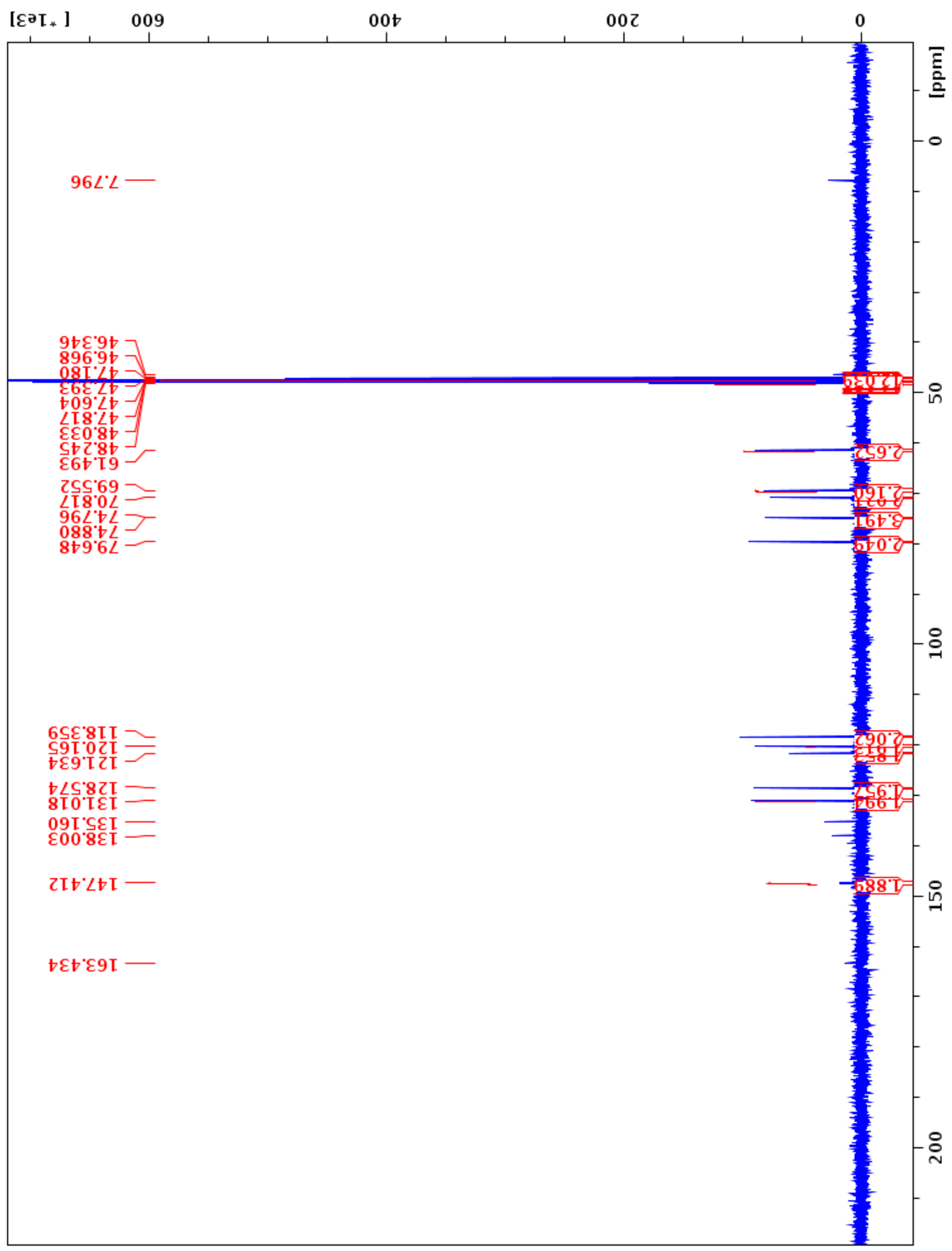


1f: 1 -(4-methylphenyl)-4-(1-deoxy- $\beta$-D-galactopyranosyl)triazole

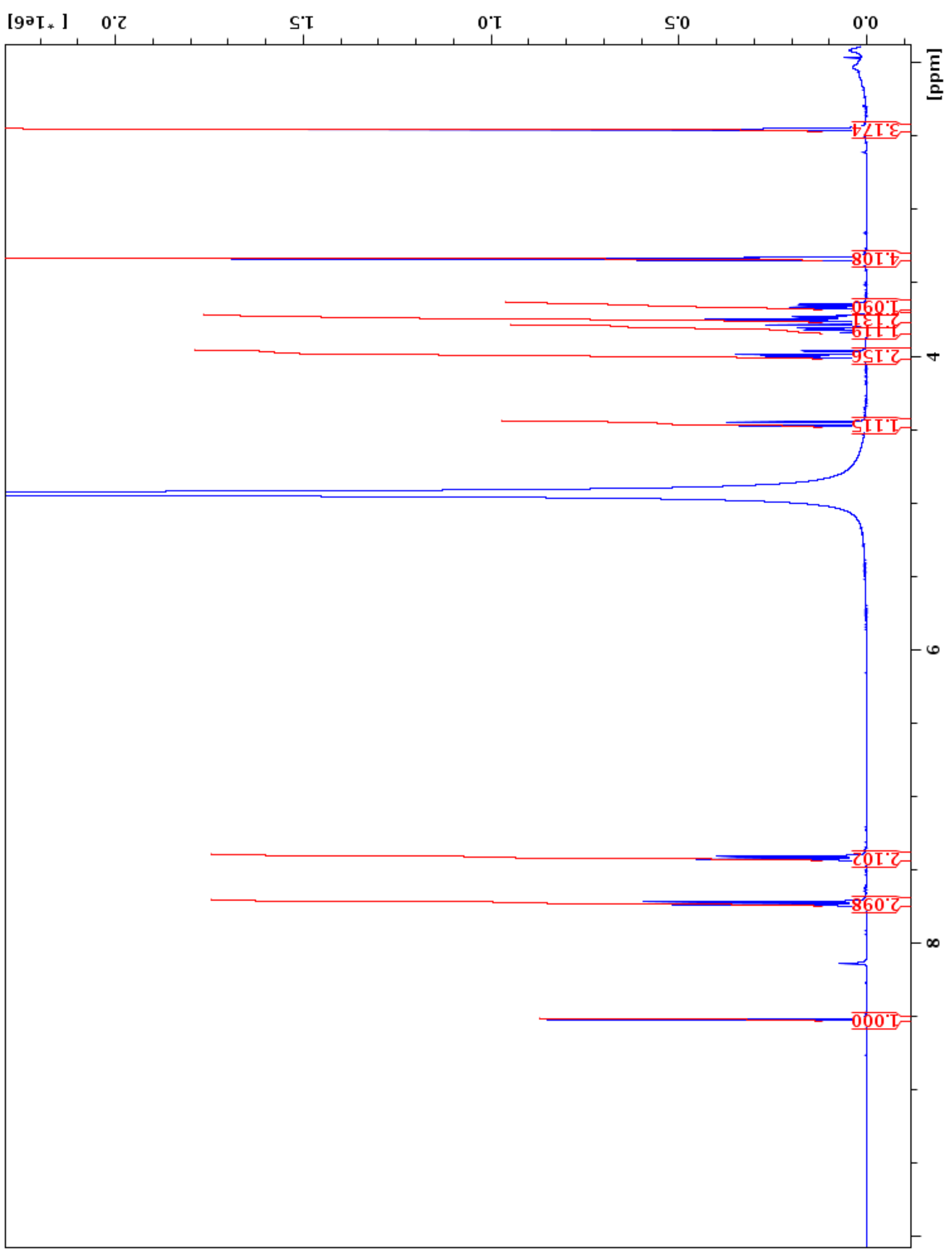


1f: 1 -(4-methylphenyl)-4-(1-deoxy- $\beta$-D-galactopyranosyl)triazole

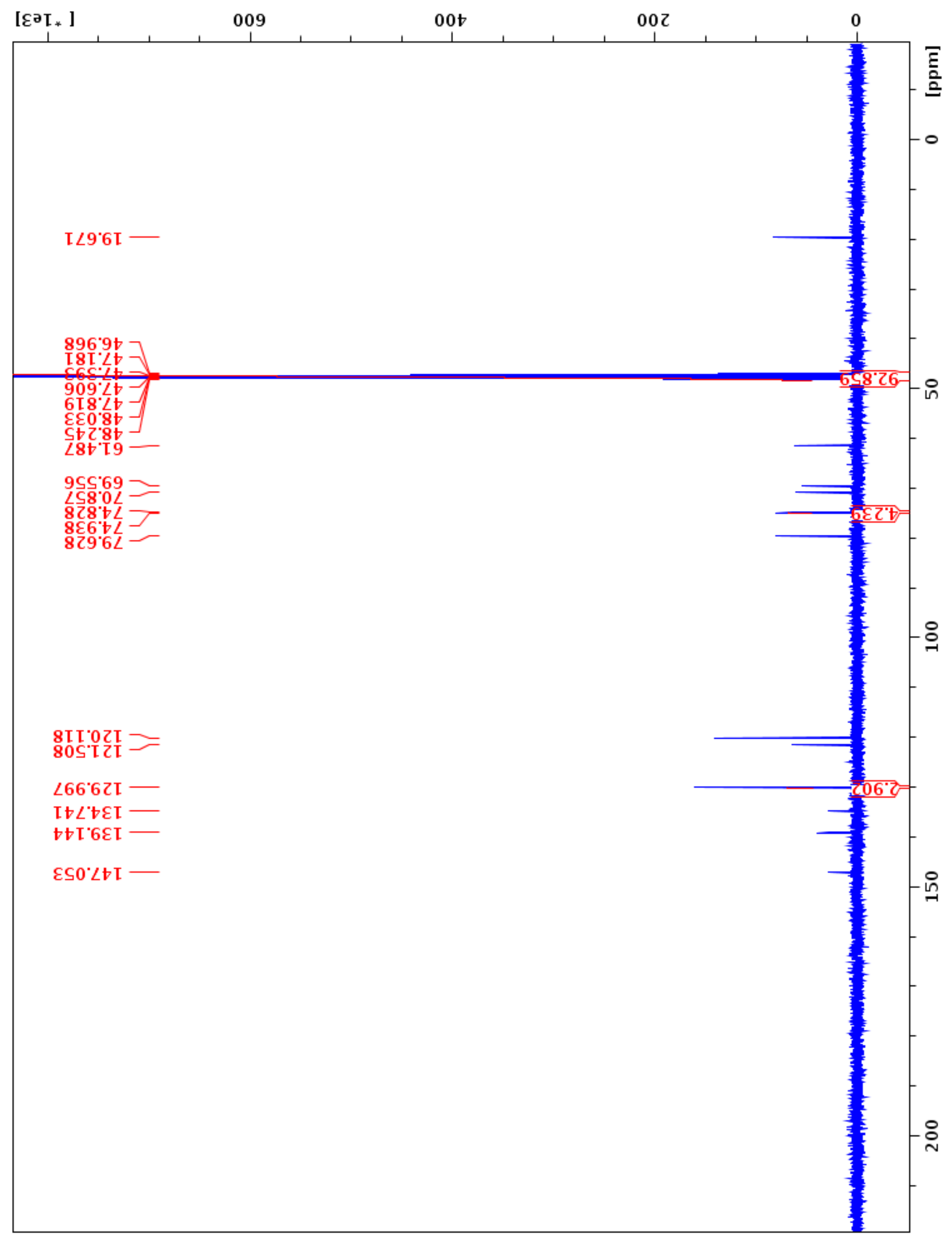


1g: 1-(3-methylphenyl)-4-(1-deoxy- $\beta$-D-galactopyranosyl)triazole

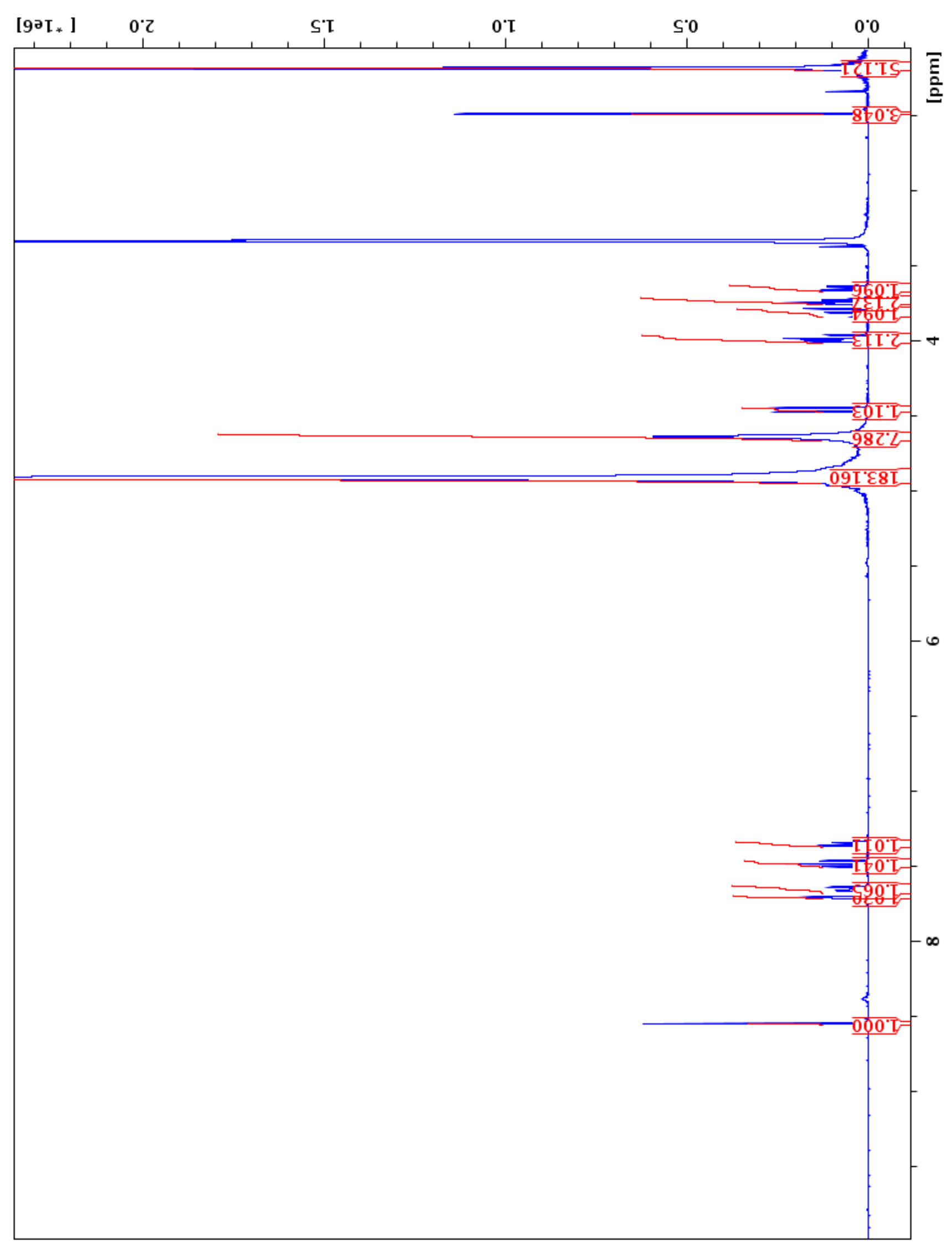


1g: 1-(3-methylphenyl)-4-(1-deoxy- $\beta$-D-galactopyranosyl)oxazole

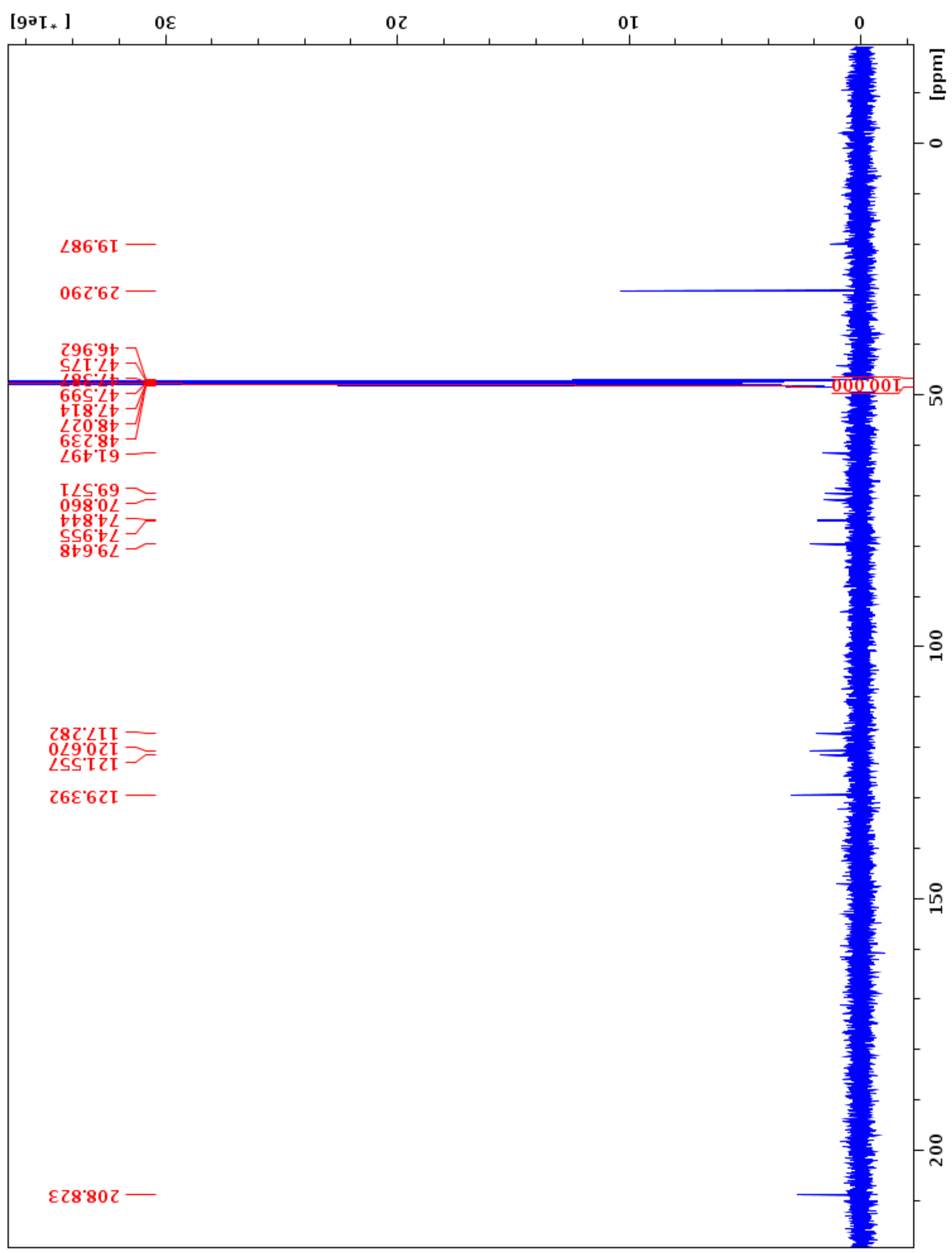


1h: 1-(4-fluorophenyl)-4-(1-deoxy- $\beta$-D-galactopyranosyl)triazole

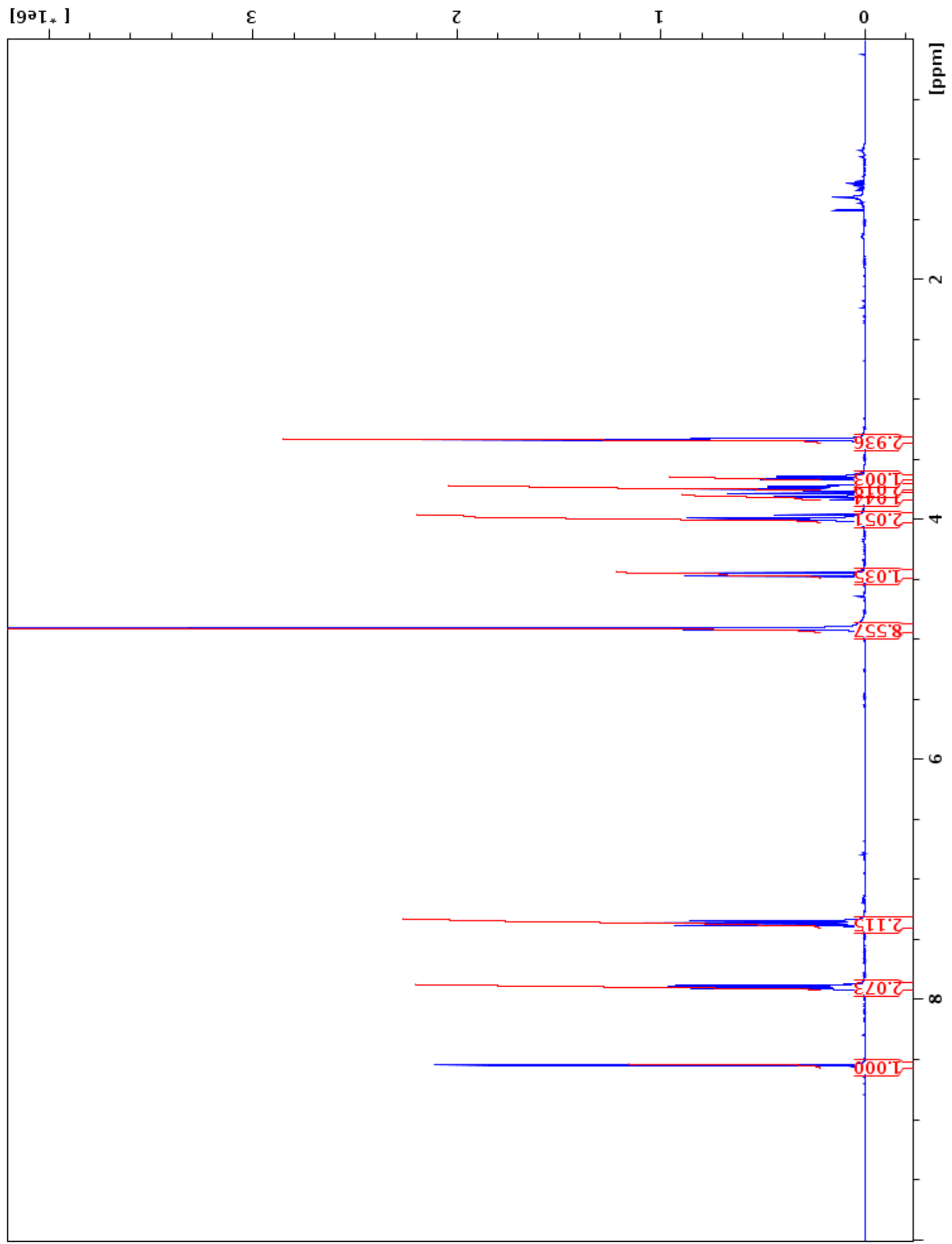


1h: 1-(4-fluorophenyl)-4-(1-deoxy- $\beta$-D-galactopyranosyl)triazole

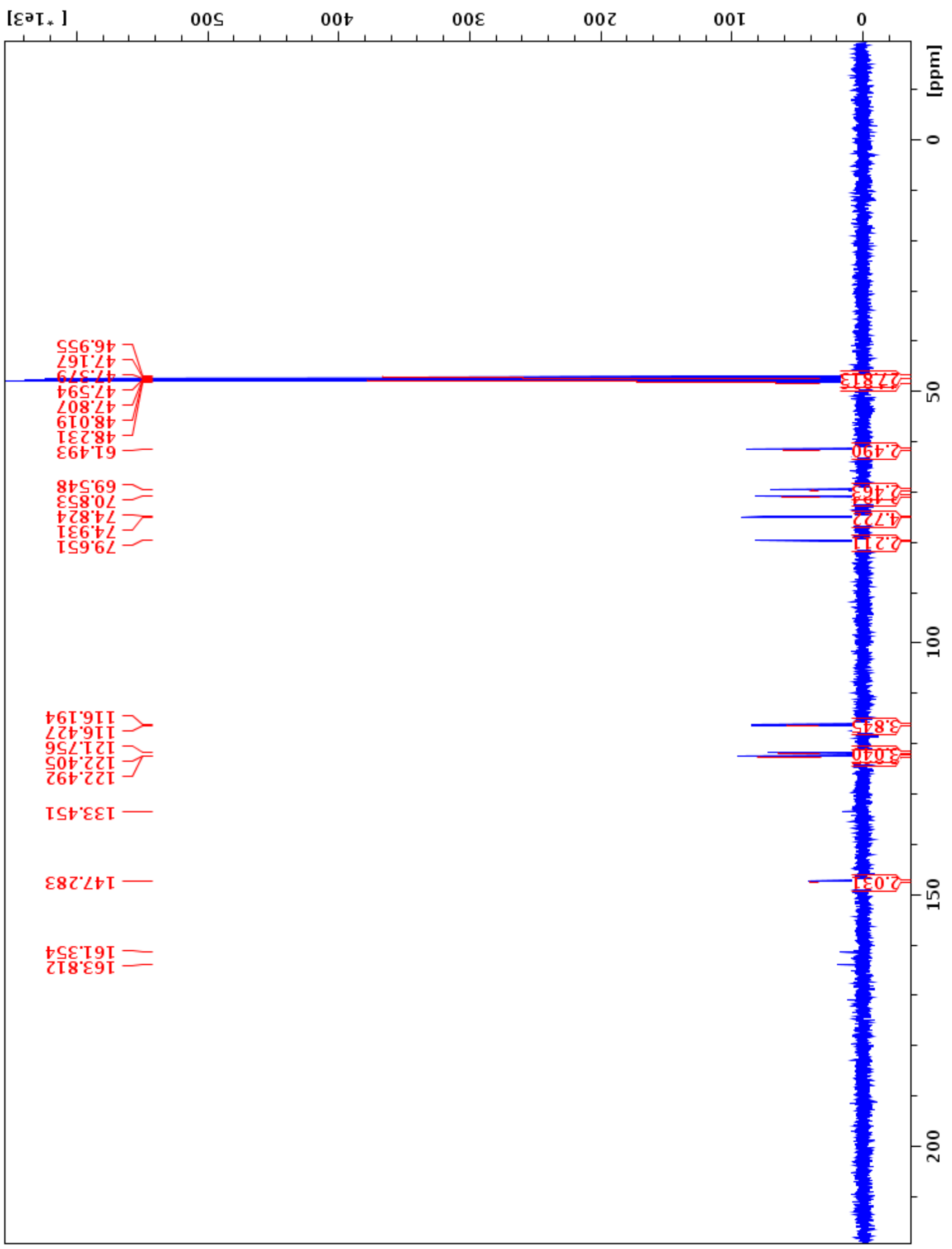


1i: 1 -(3-fluorophenyl)-4-(1-deoxy- $\beta$-D-galactopyranosyl)triazole

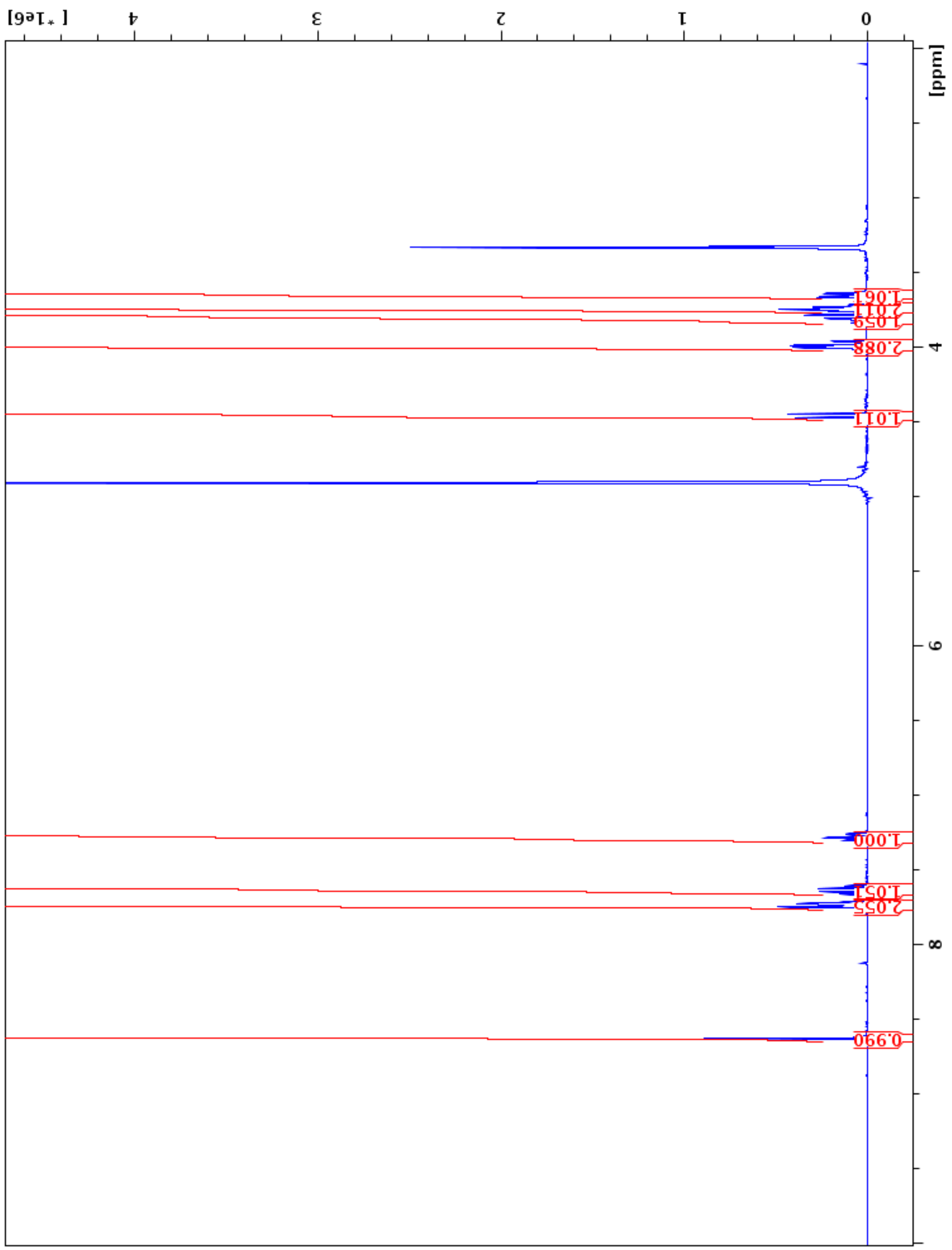


1i: 1-(3-fluorophenyl)-4-(1-deoxy- $\beta$-D-galactopyranosyl)triazole

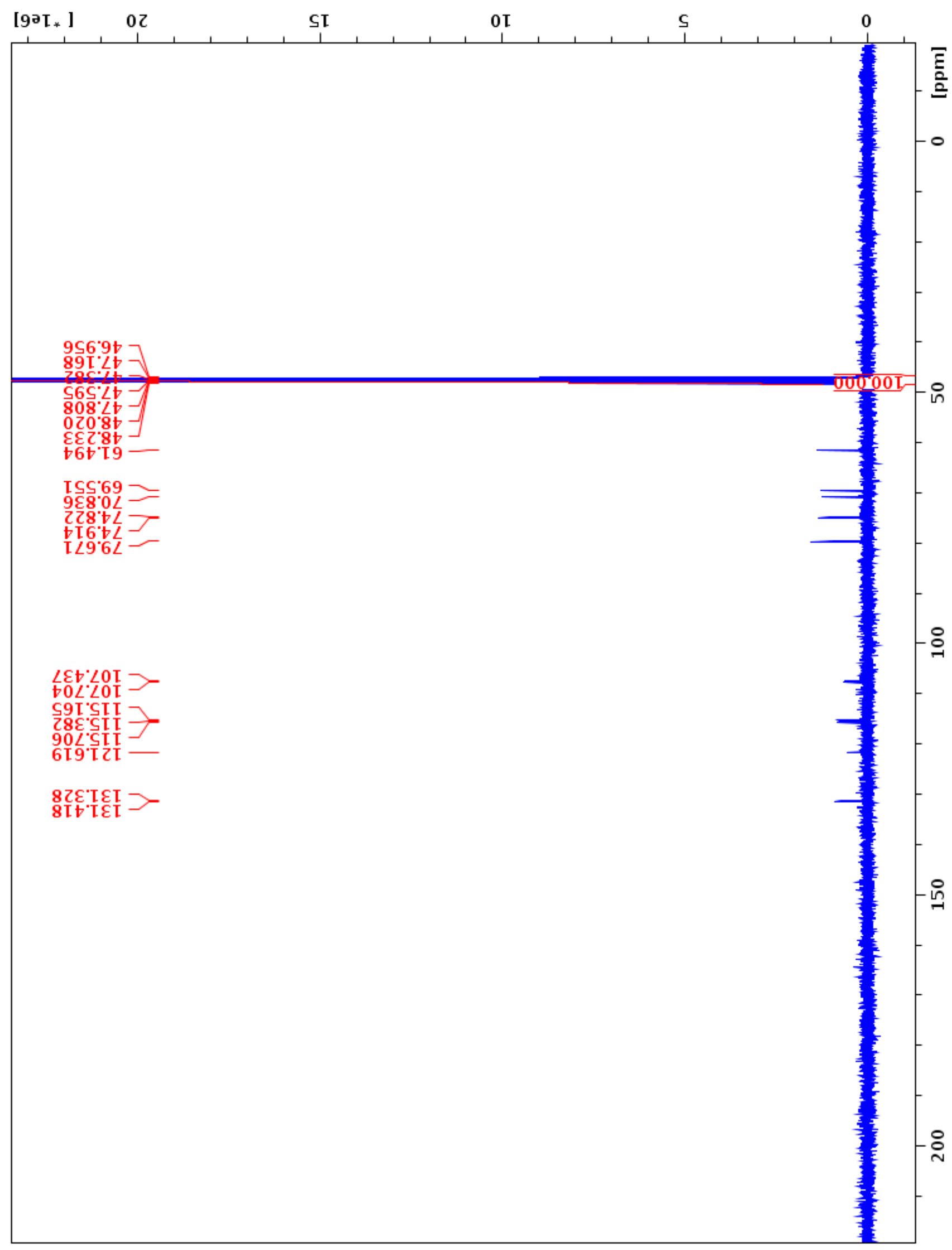


1j: 1-(2-fluorophenyl)-4-(1-deoxy- $\beta$-D-galactopyranosyl)triazole

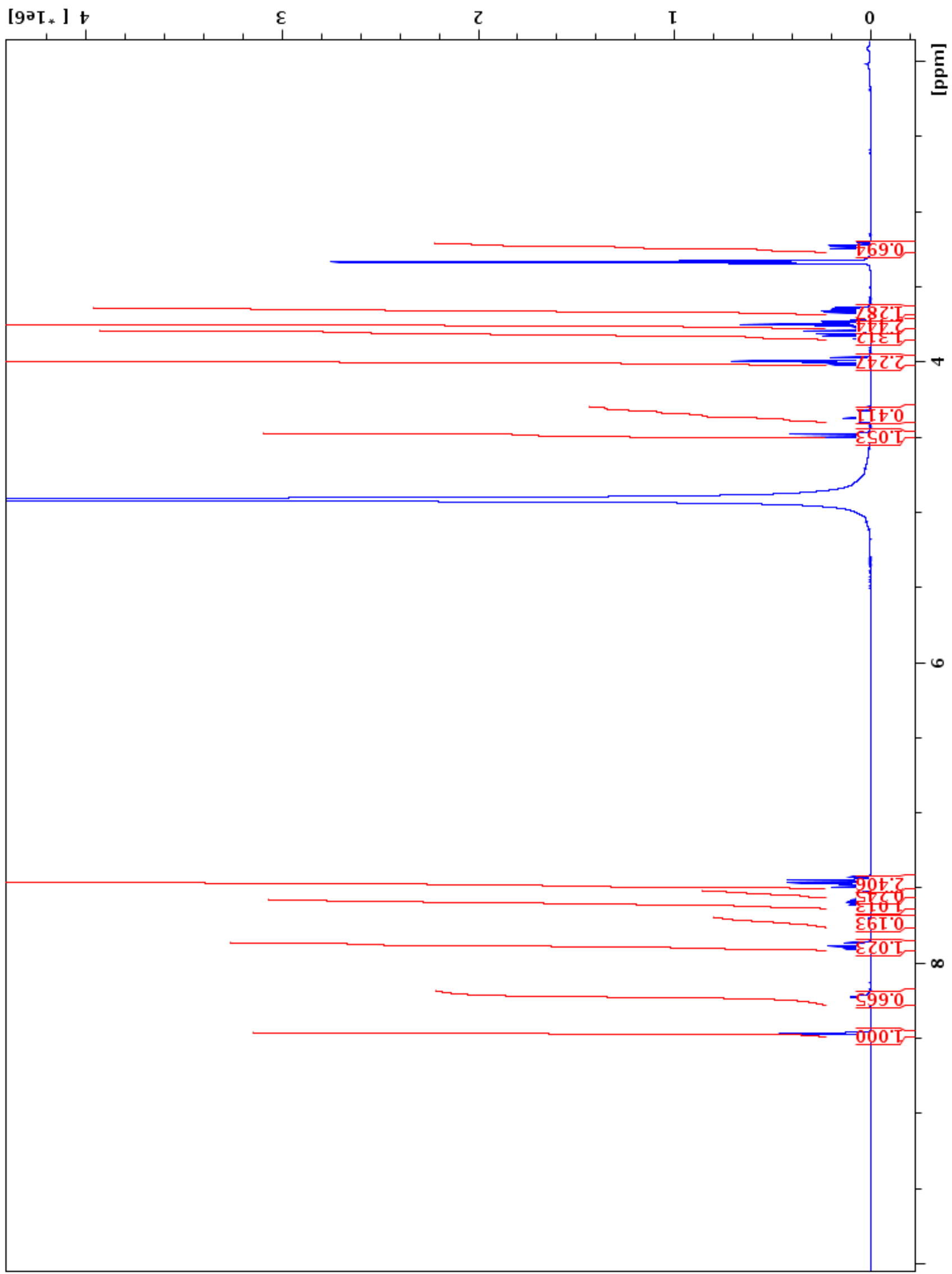


1j: 1-(2-fluorophenyl)-4-(1-deoxy- $\beta$-D-galactopyranosyl)triazole

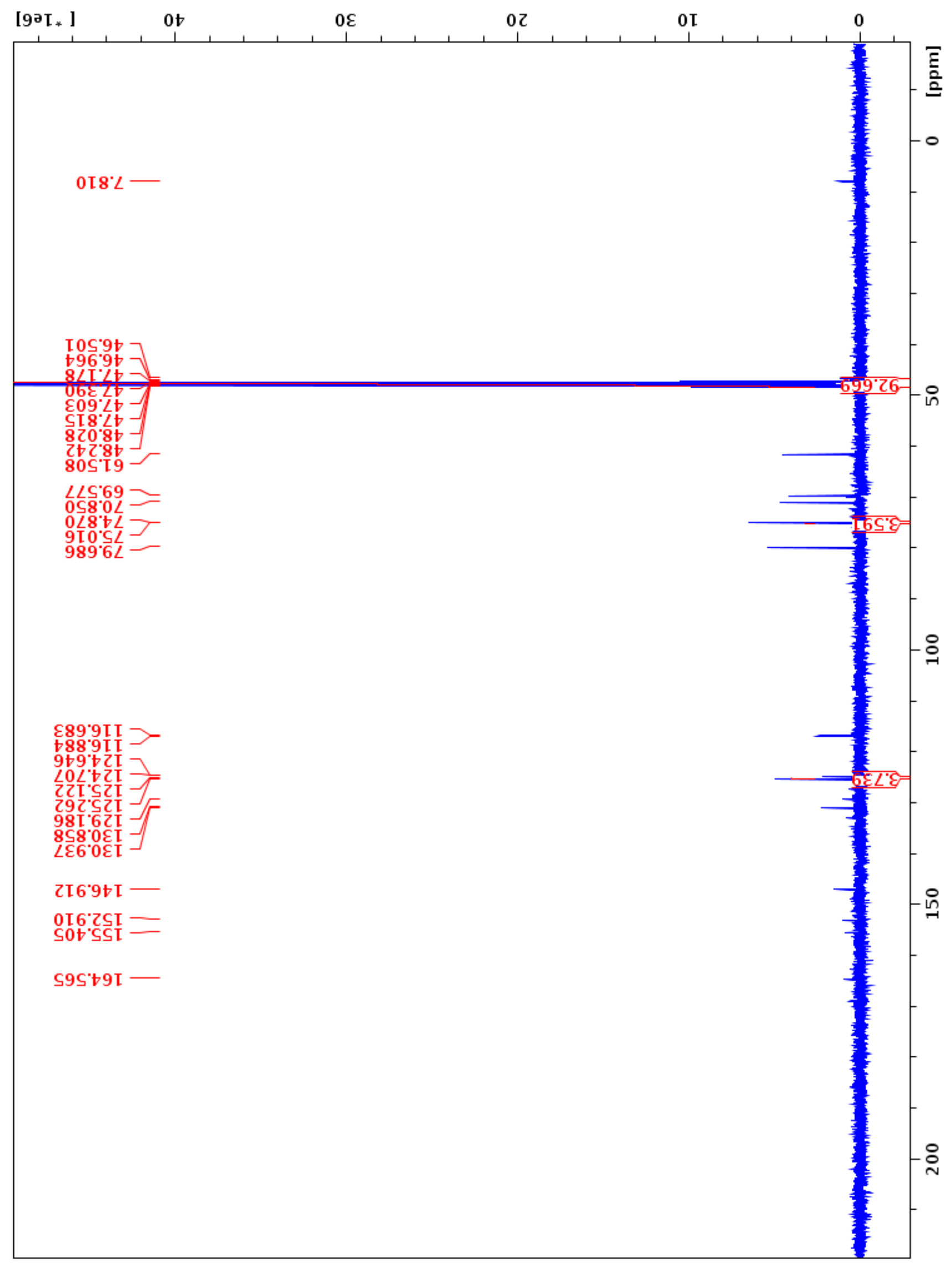


2a: 2-phenyl-5-(1-deoxy- $\beta$-D-galactopyranosyl)oxazole

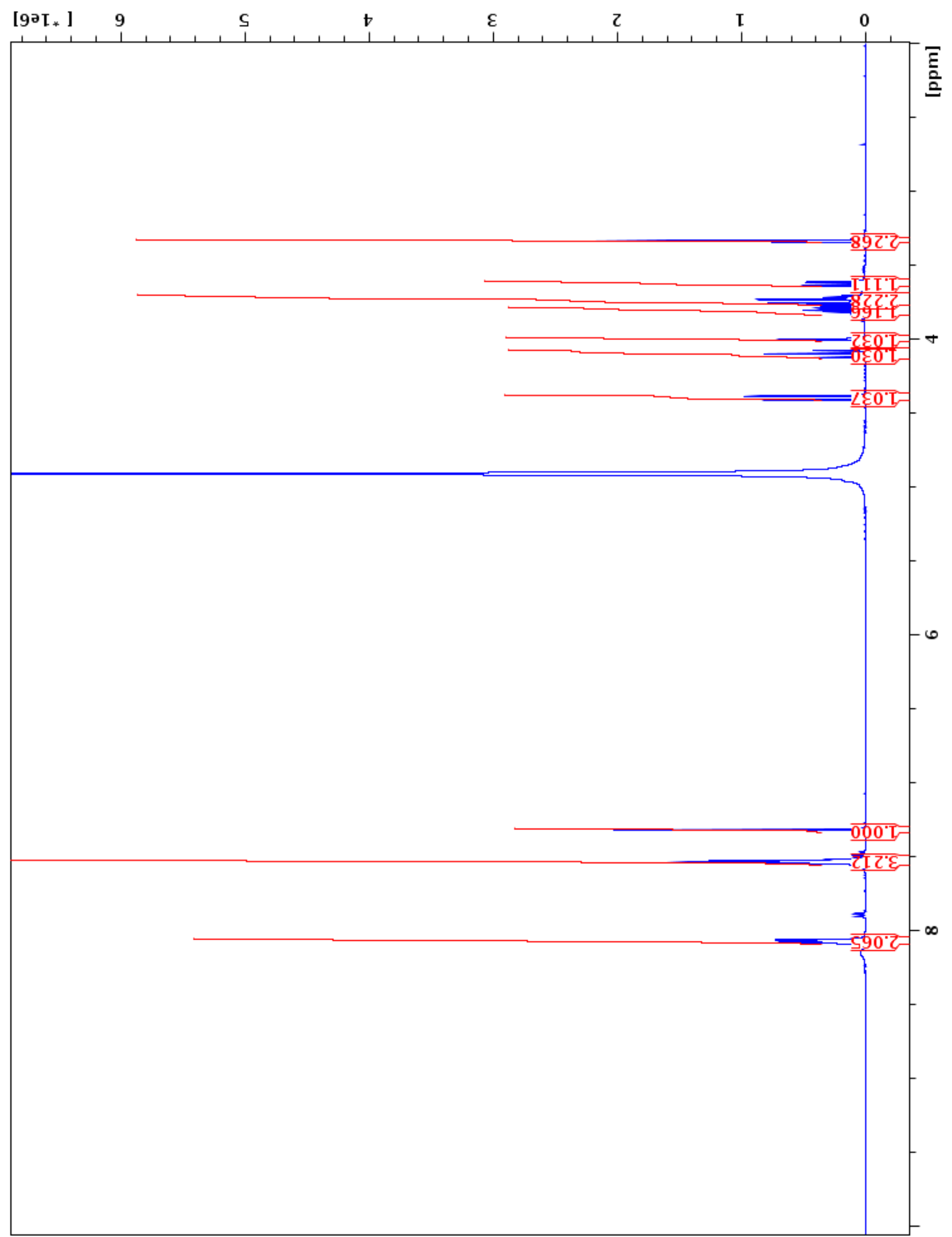


2a: 2-phenyl-5-(1-deoxy- $\beta$-D-galactopyranosyl)oxazole

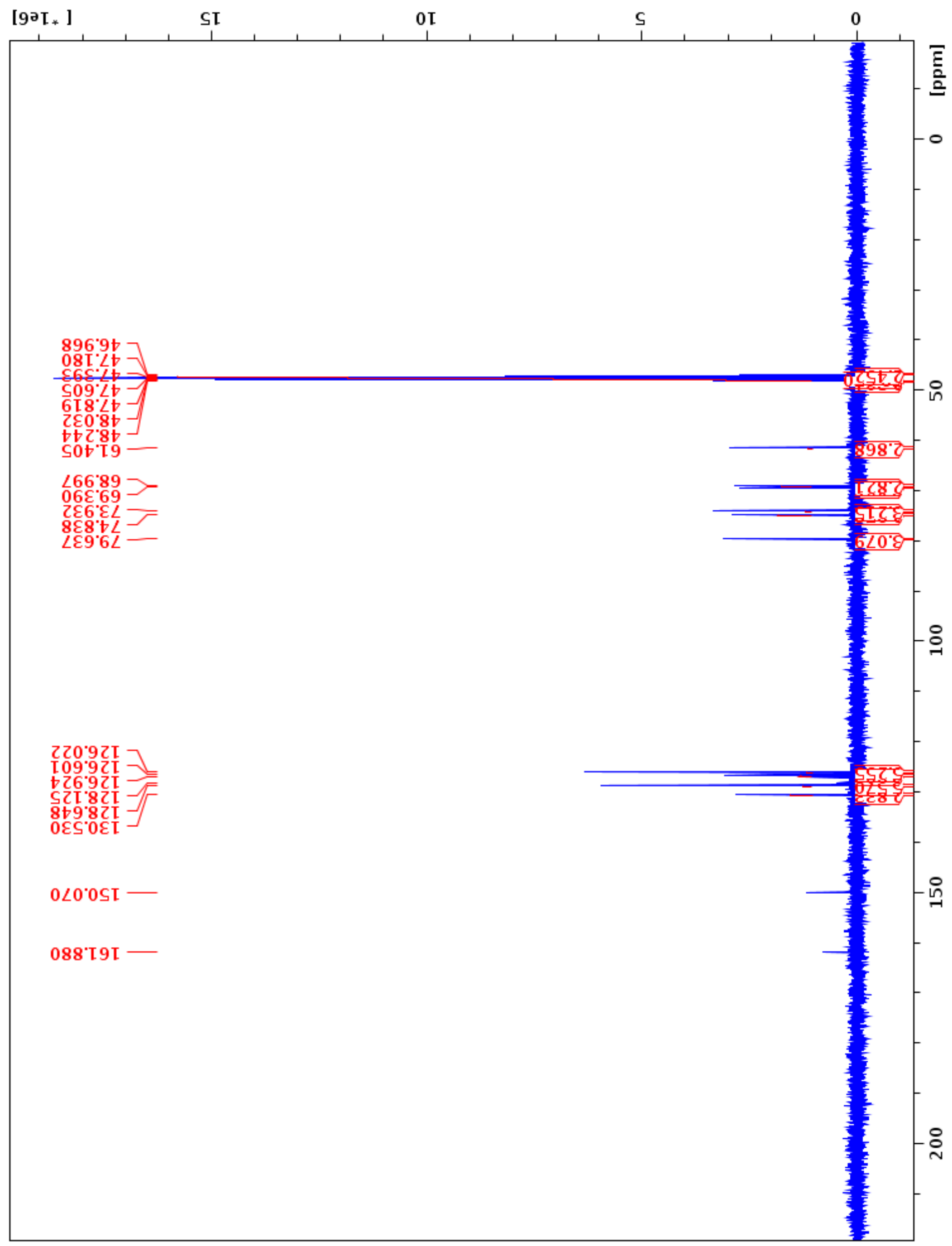


2b: 2-naphth-1-yl-5-(1-deoxy- $\beta$-D-galactopyranosyl)oxazole

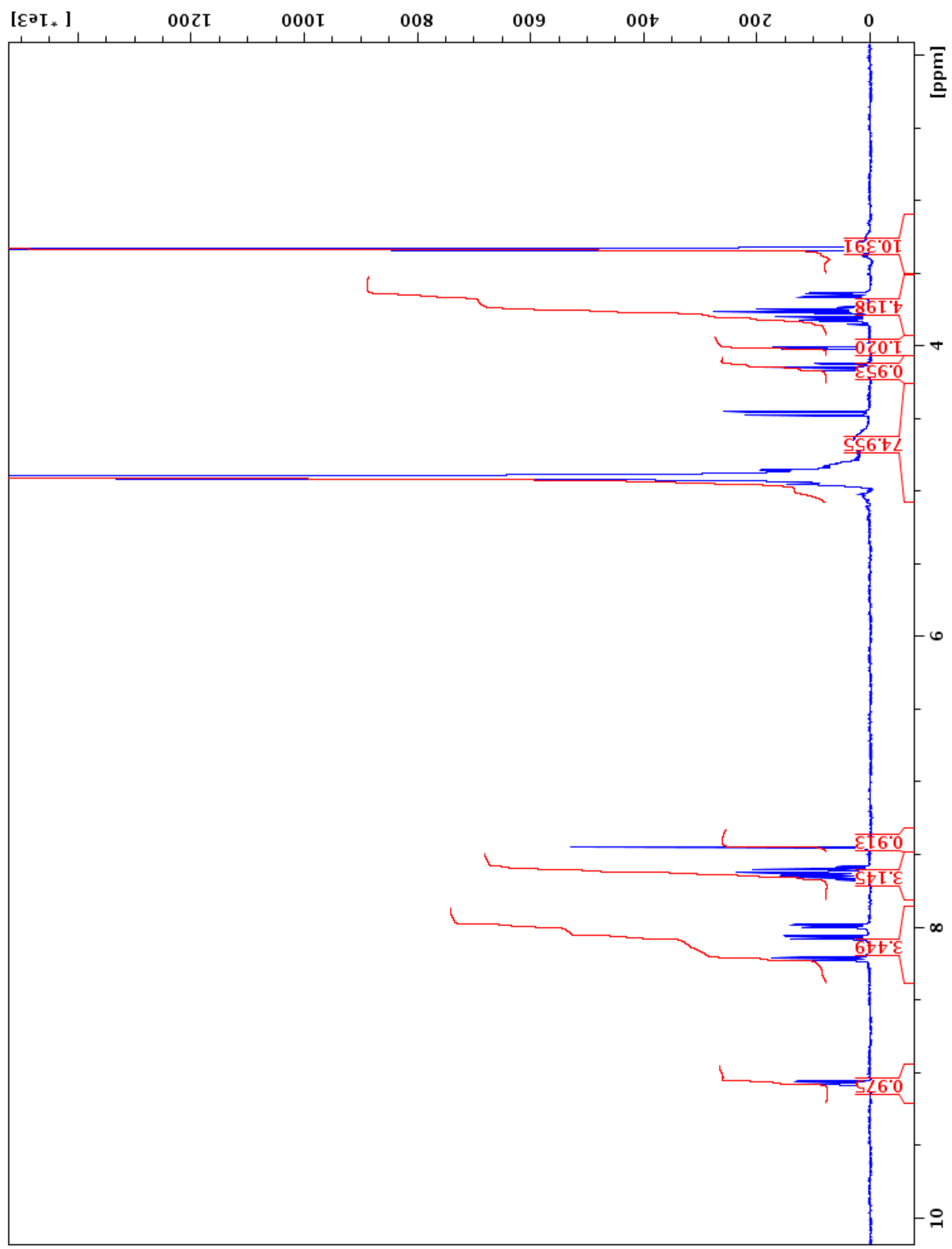


2b: 2-naphth-1-yl-5-(1-deoxy- $\beta$-D-galactopyranosyl)oxazole

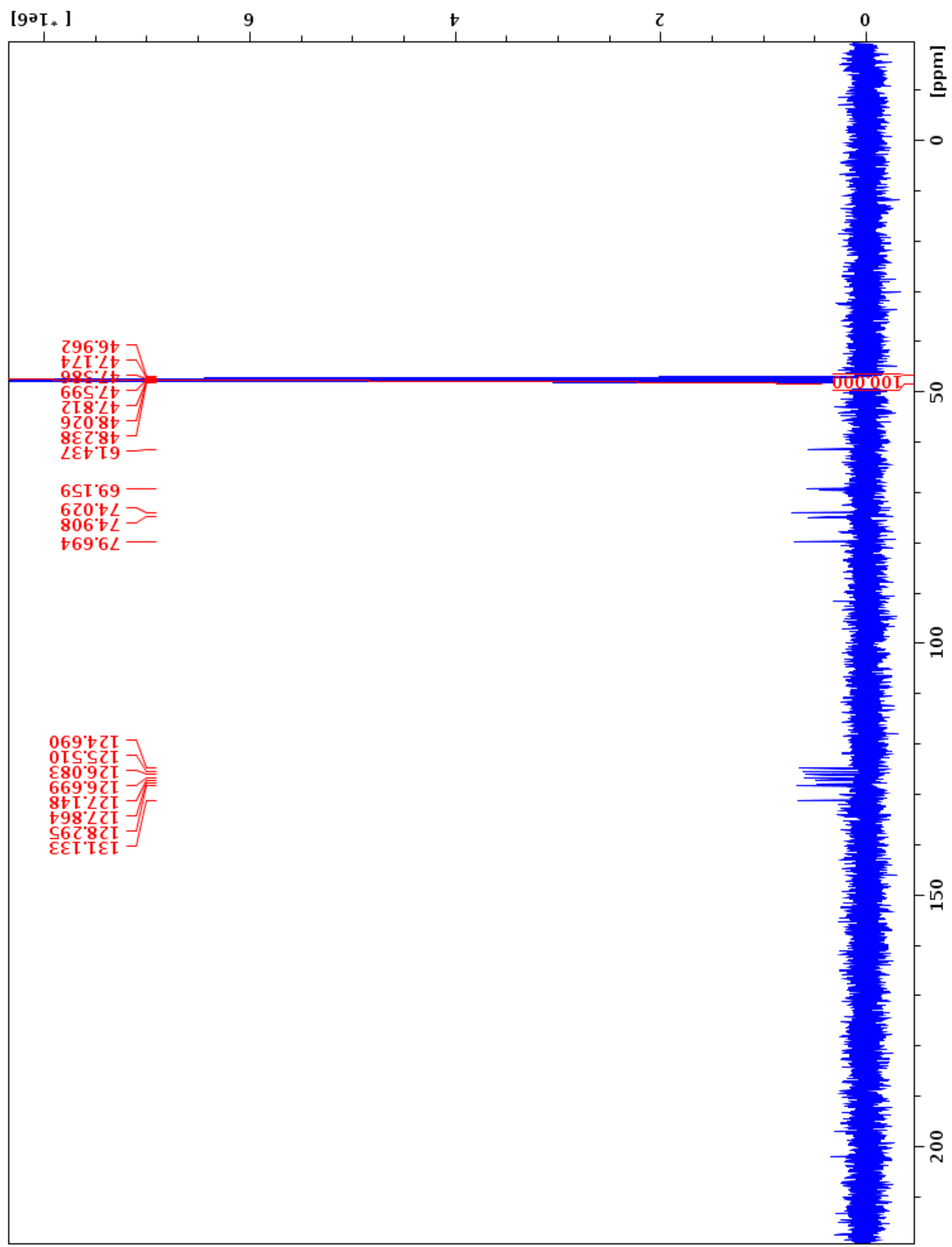


2c: 2-naphth-2-yl-5-(1-deoxy- $\beta$-D-galactopyranosyl)oxazole

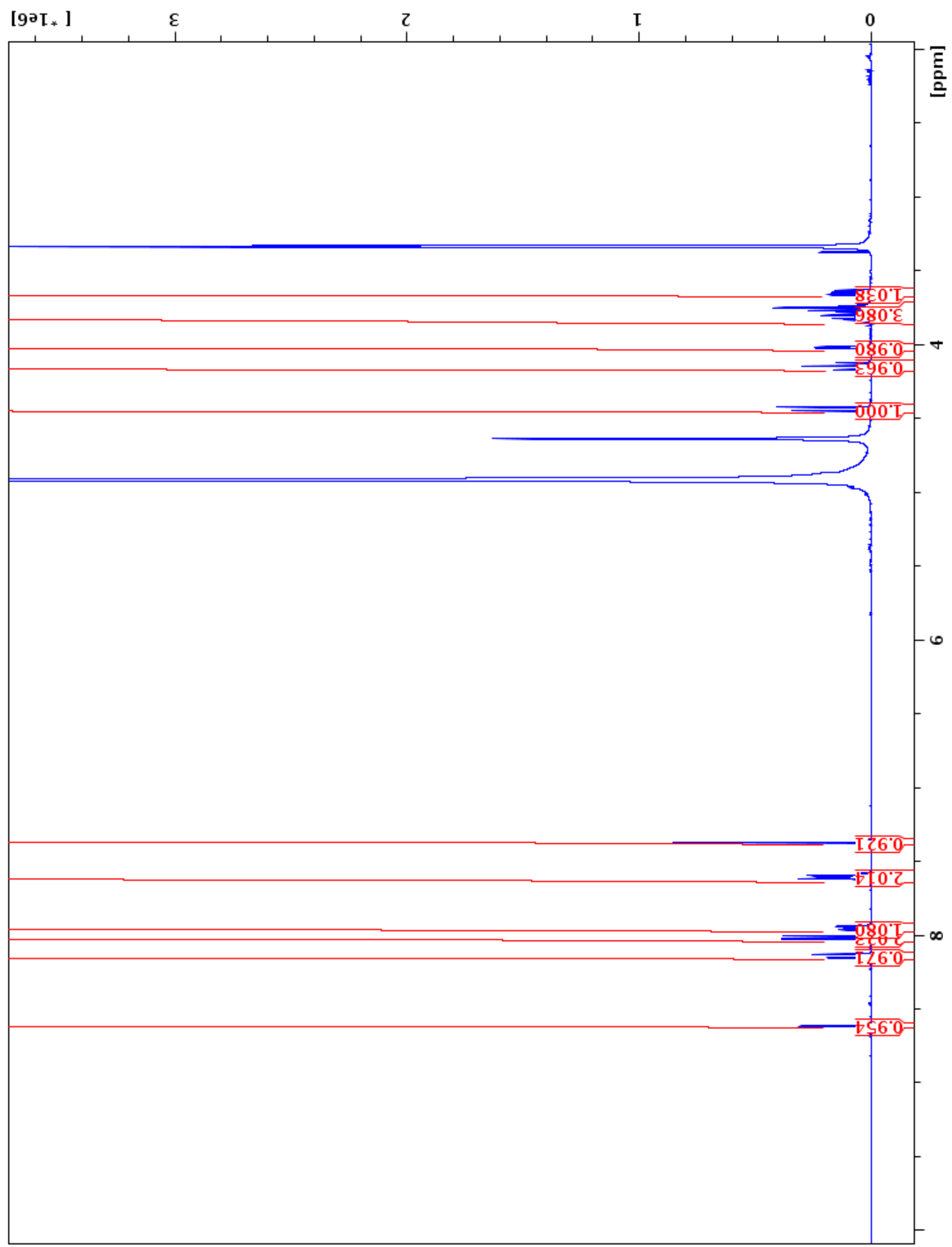


2c: 2-naphth-2-yl-5-(1-deoxy- $\beta$-D-galactopyranosyl)oxazole

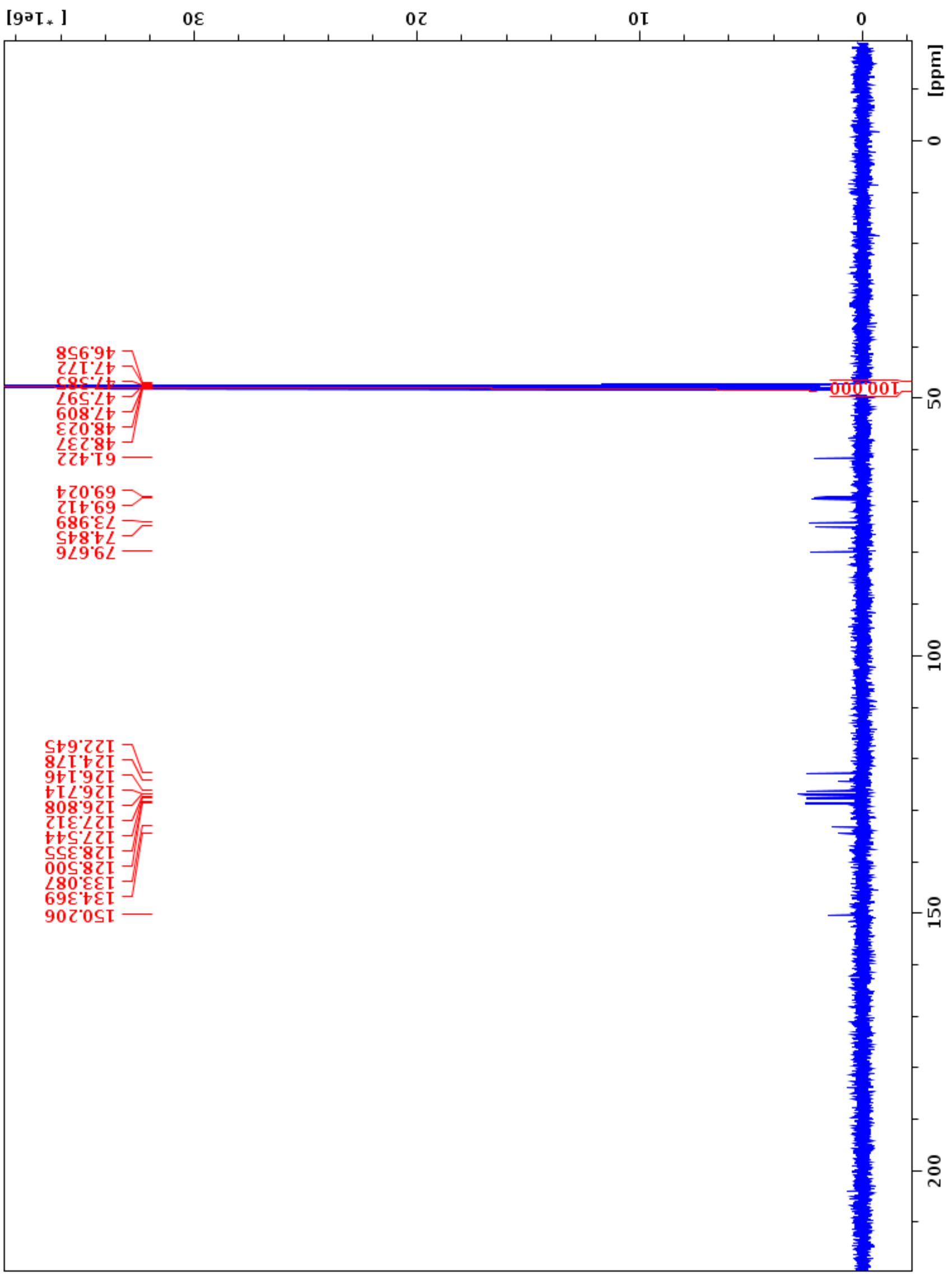


2d: 2-(4-chlorophenyl)-5-(1-deoxy- $\beta$-D-galactopyranosyl)oxazole

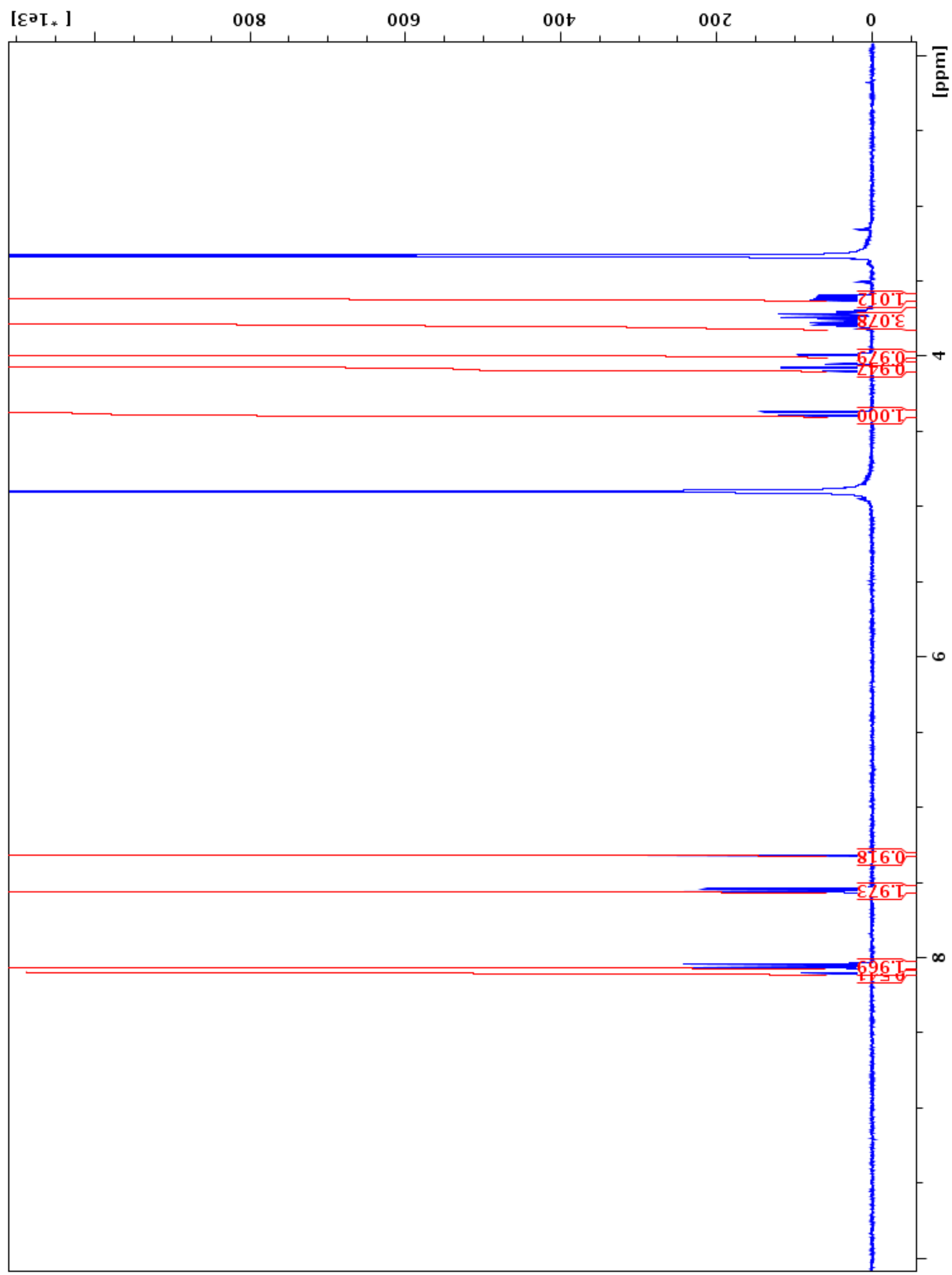


2d: 2-(4-chlorophenyl)-5-(1-deoxy- $\beta$-D-galactopyranosyl)oxazole

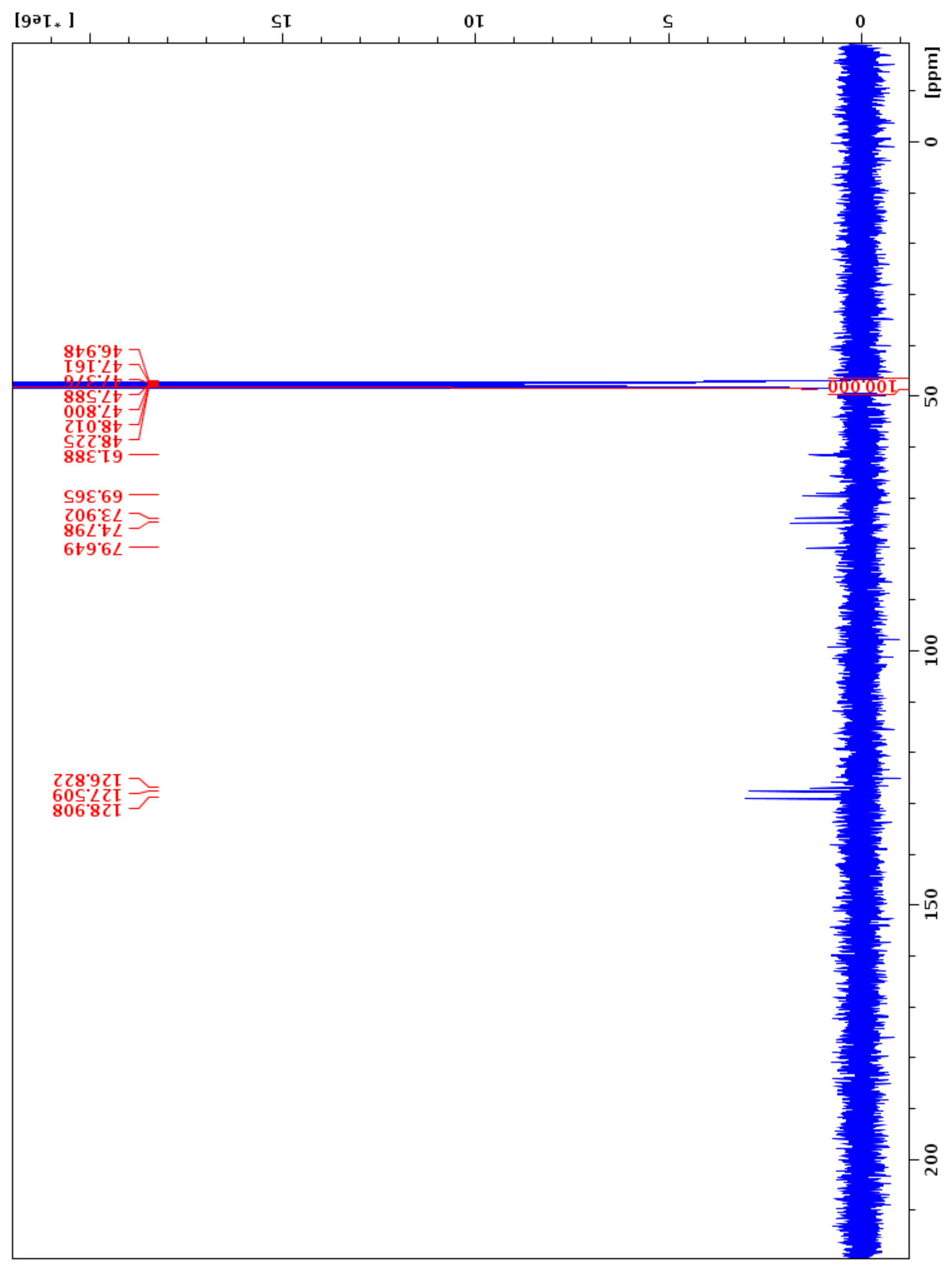


2e: 2-(3-chlorophenyl)-5-(1-deoxy- $\beta$-D-galactopyranosyl)oxazole

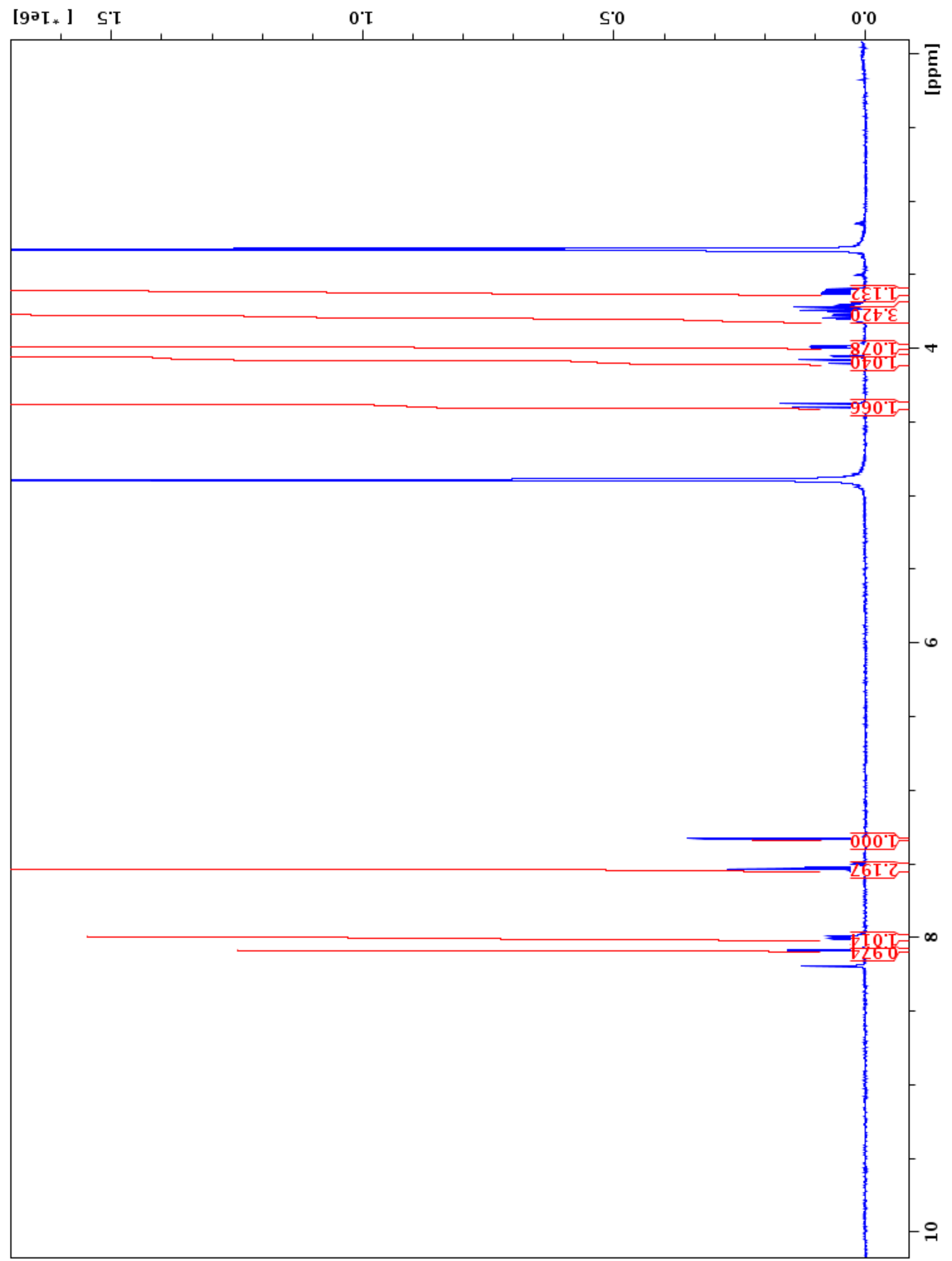


2e: 2-(3-chlorophenyl)-5-(1-deoxy- $\beta$-D-galactopyranosyl)oxazole

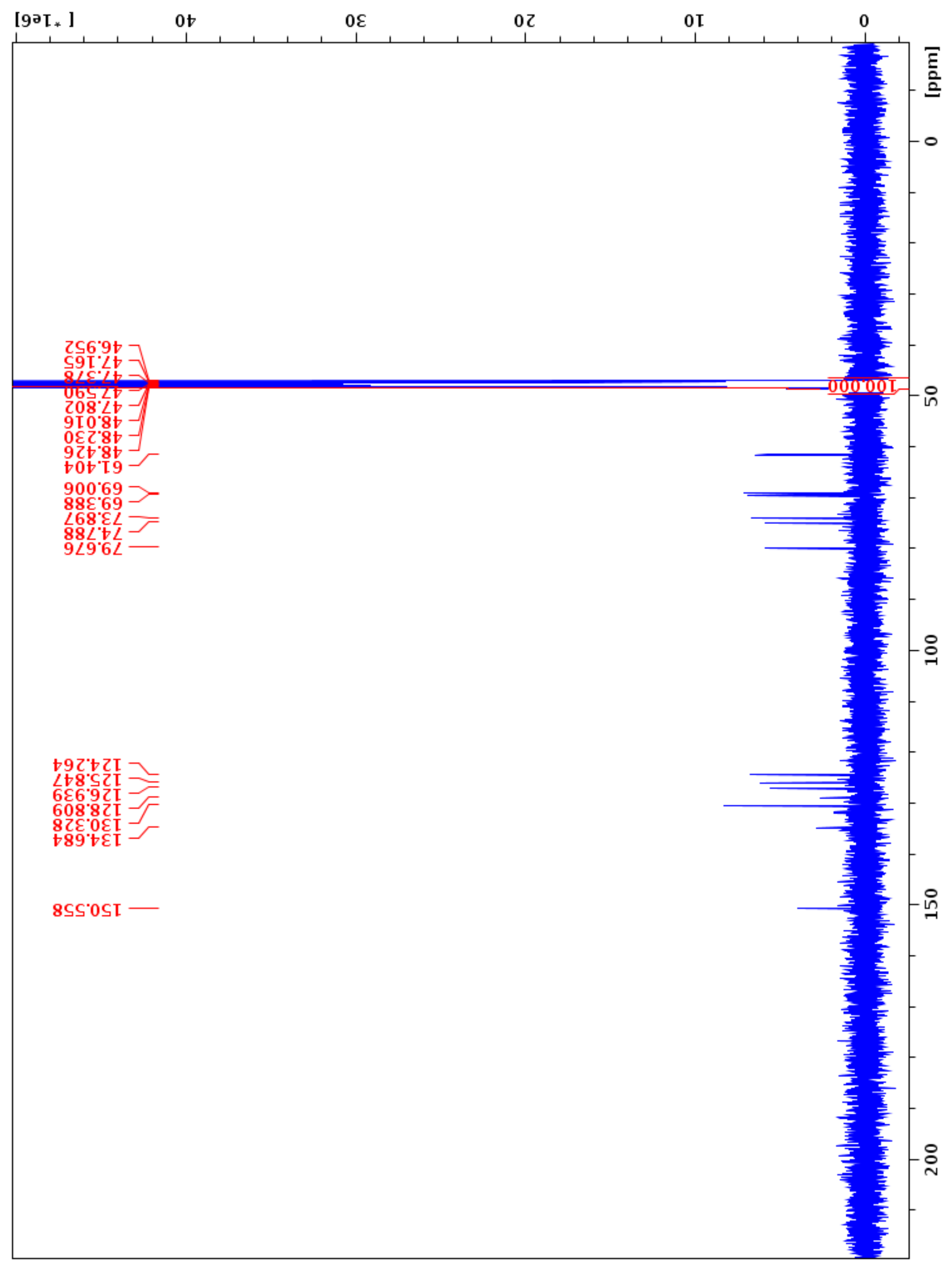


2f: 2-(4-methylphenyl)-5-(1-deoxy- $\beta$-D-galactopyranosyl)oxazole

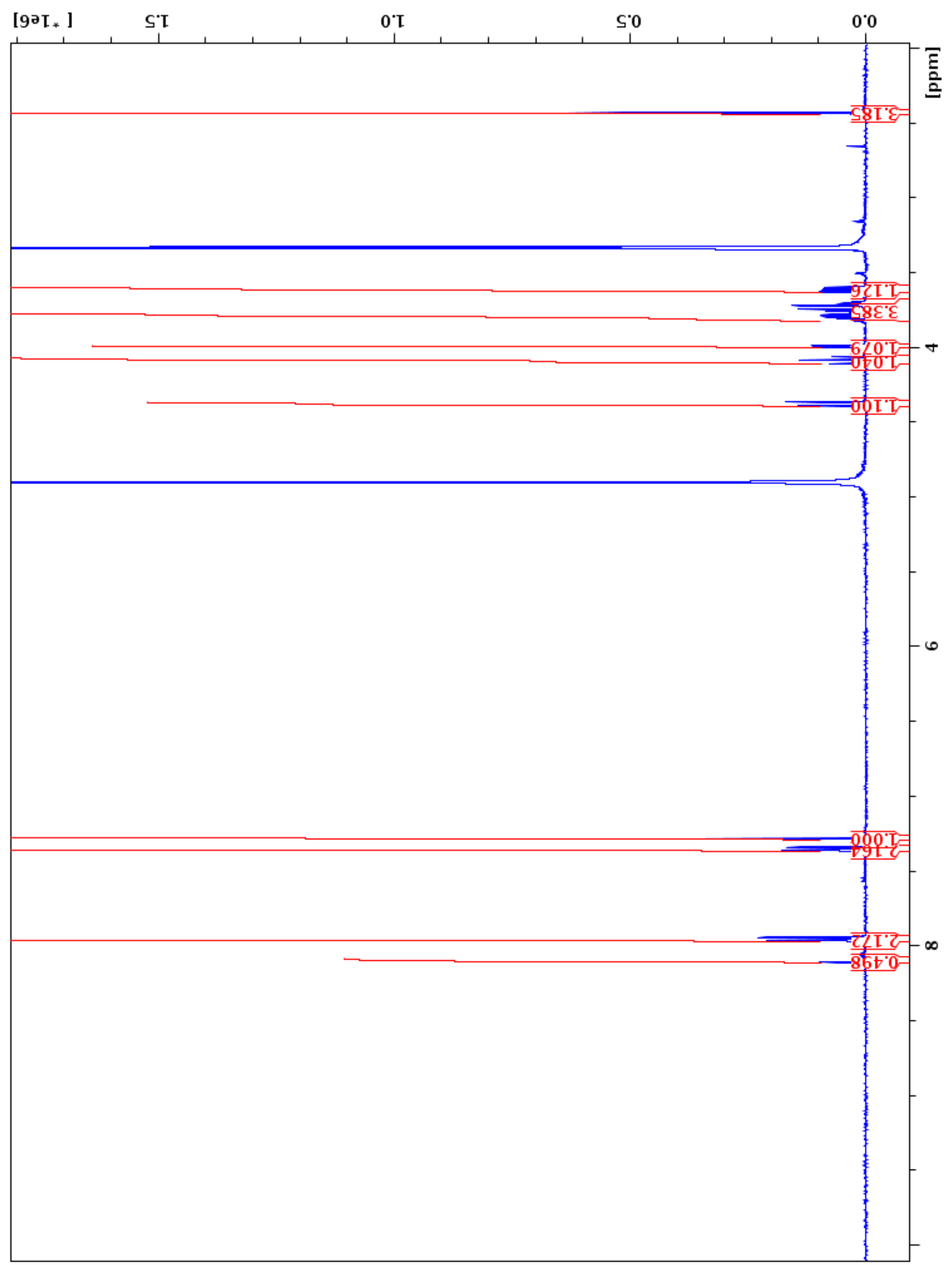


2f: 2-(4-methylphenyl)-5-(1-deoxy- $\beta$-D-galactopyranosyl)oxazole

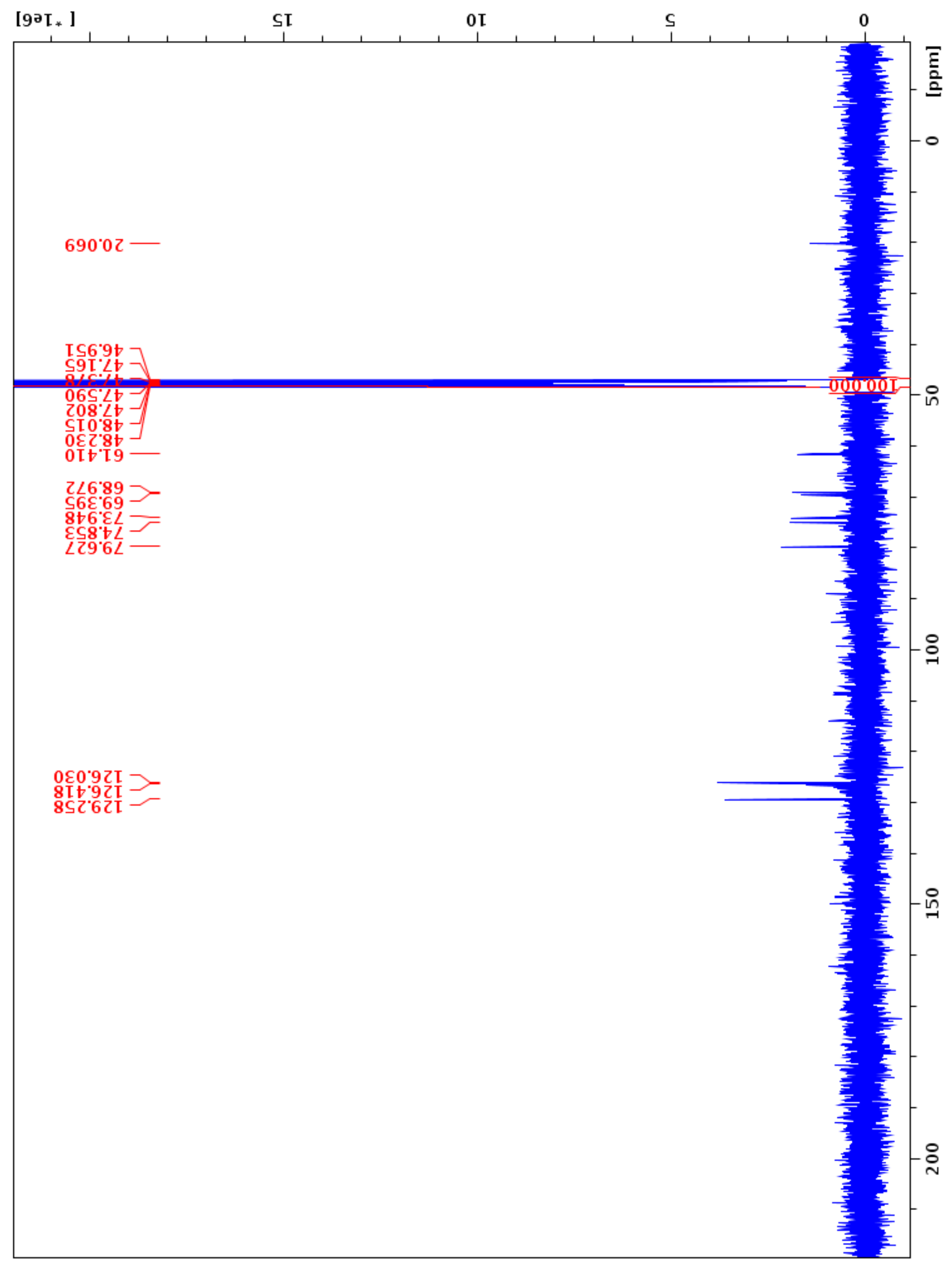


2g: 2-(3-methylphenyl)-5-(1-deoxy- $\beta$-D-galactopyranosyl)oxazole

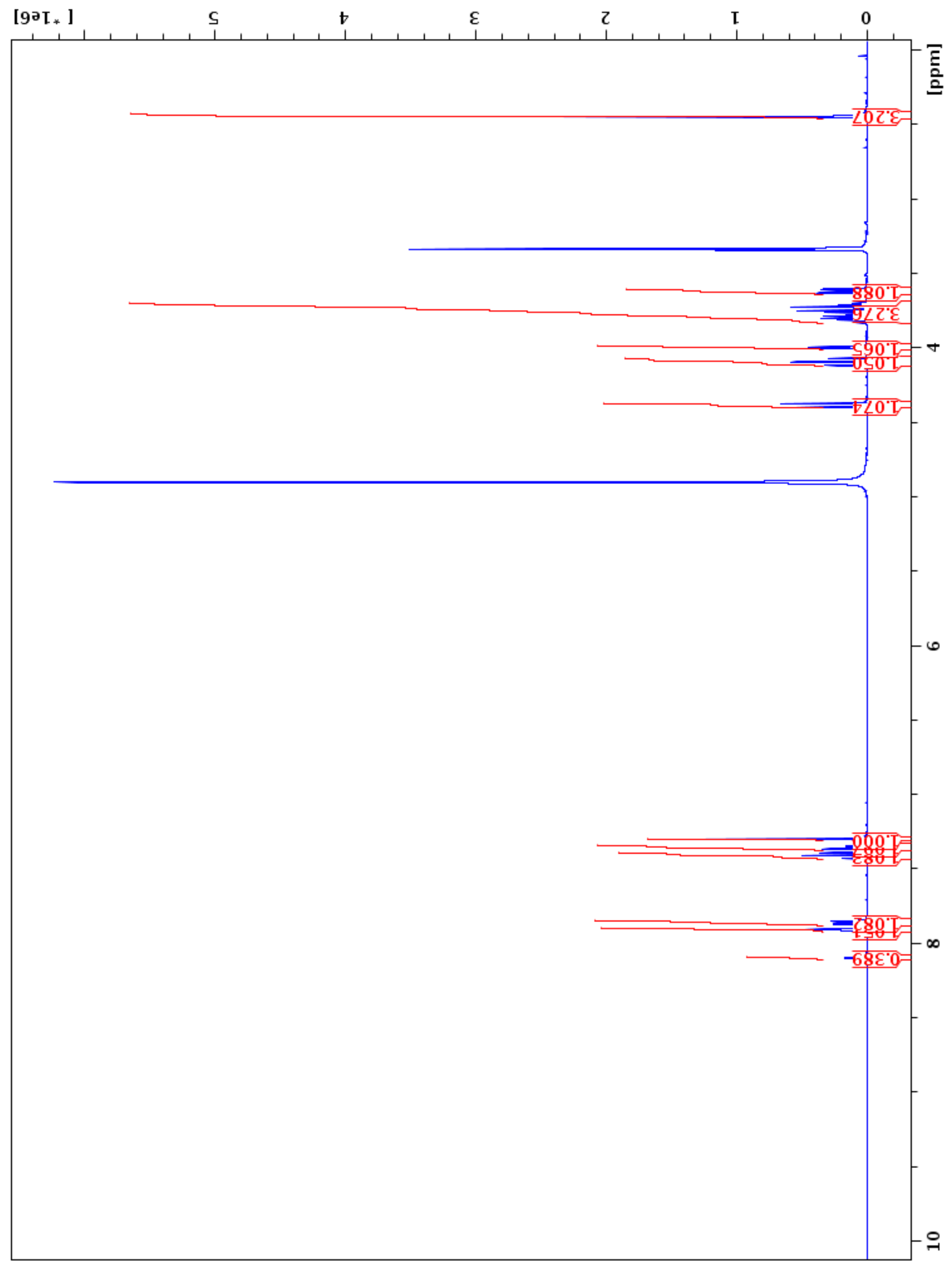


2g: 2-(3-methylphenyl)-5-(1-deoxy- $\beta$-D-galactopyranosyl)oxazole

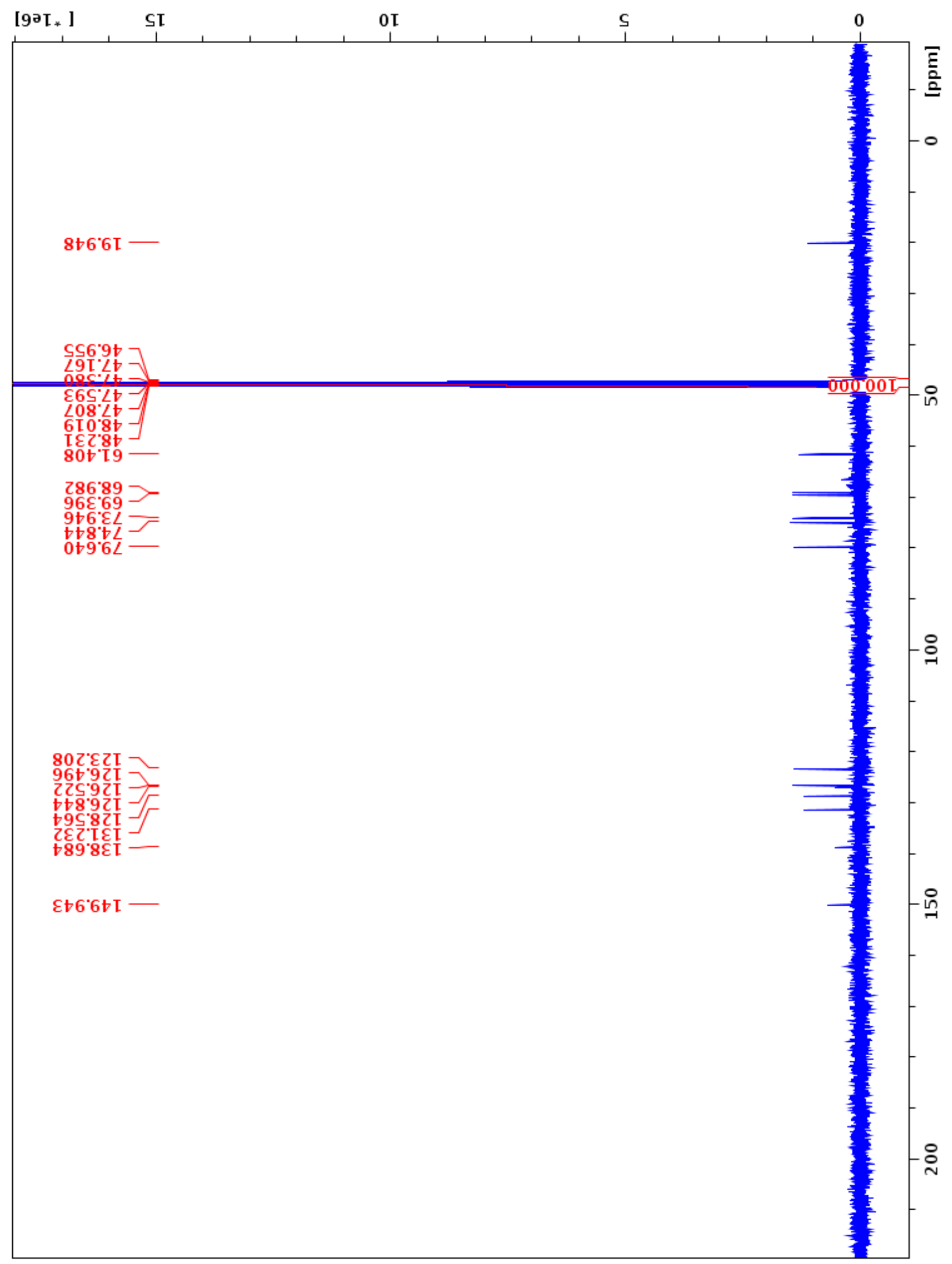


2h: 2-(4-fluorophenyl)-5-(1-deoxy- $\beta$-D-galactopyranosyl)oxazole

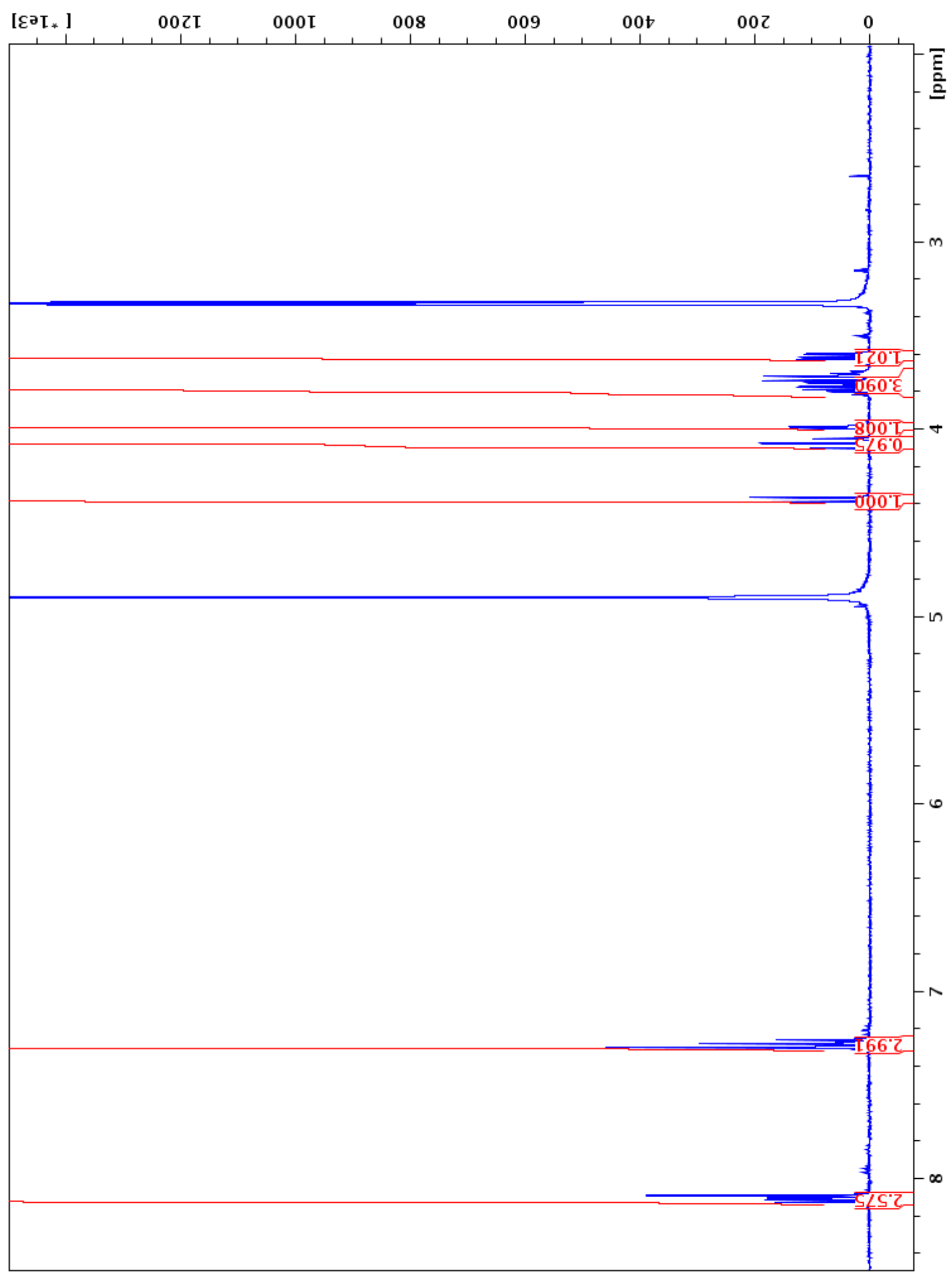


2h: 2-(4-fluorophenyl)-5-(1-deoxy- $\beta$-D-galactopyranosyl)oxazole

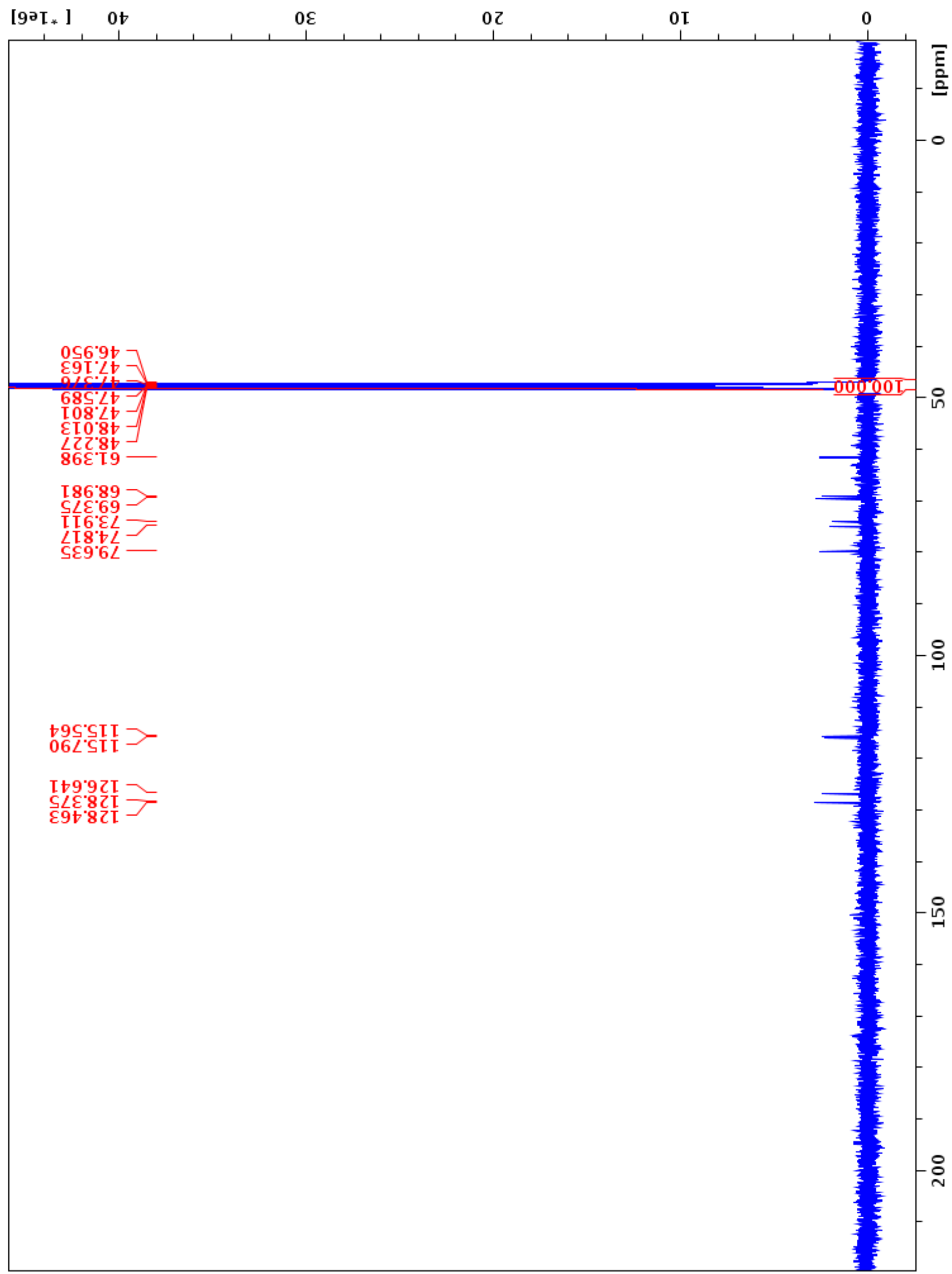


2i: 2-(3-fluorophenyl)-5-(1-deoxy- $\beta$-D-galactopyranosyl)oxazole

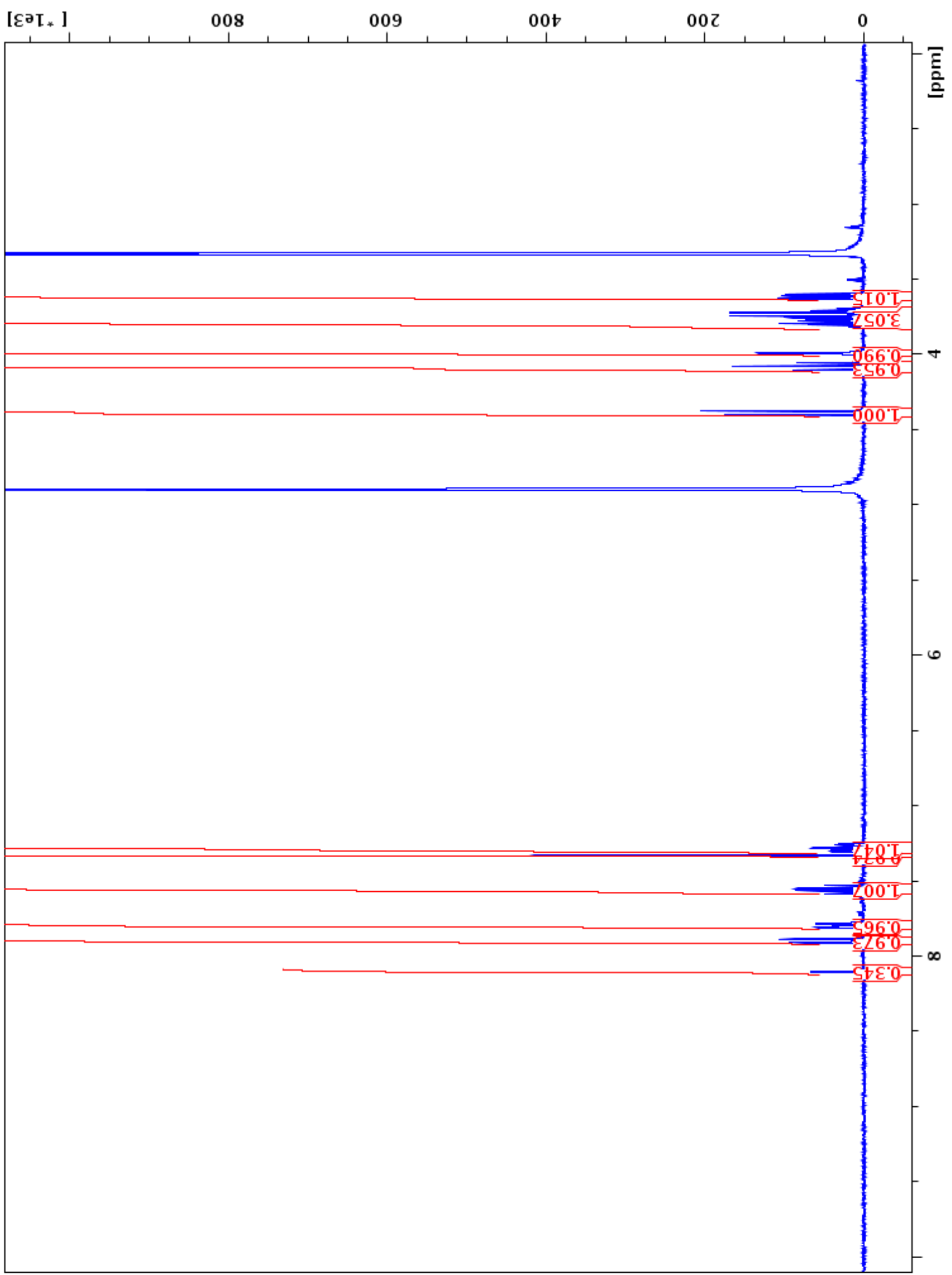


2i: 2-(3-fluorophenyl)-5-(1-deoxy- $\beta$-D-galactopranosyl)oxazole

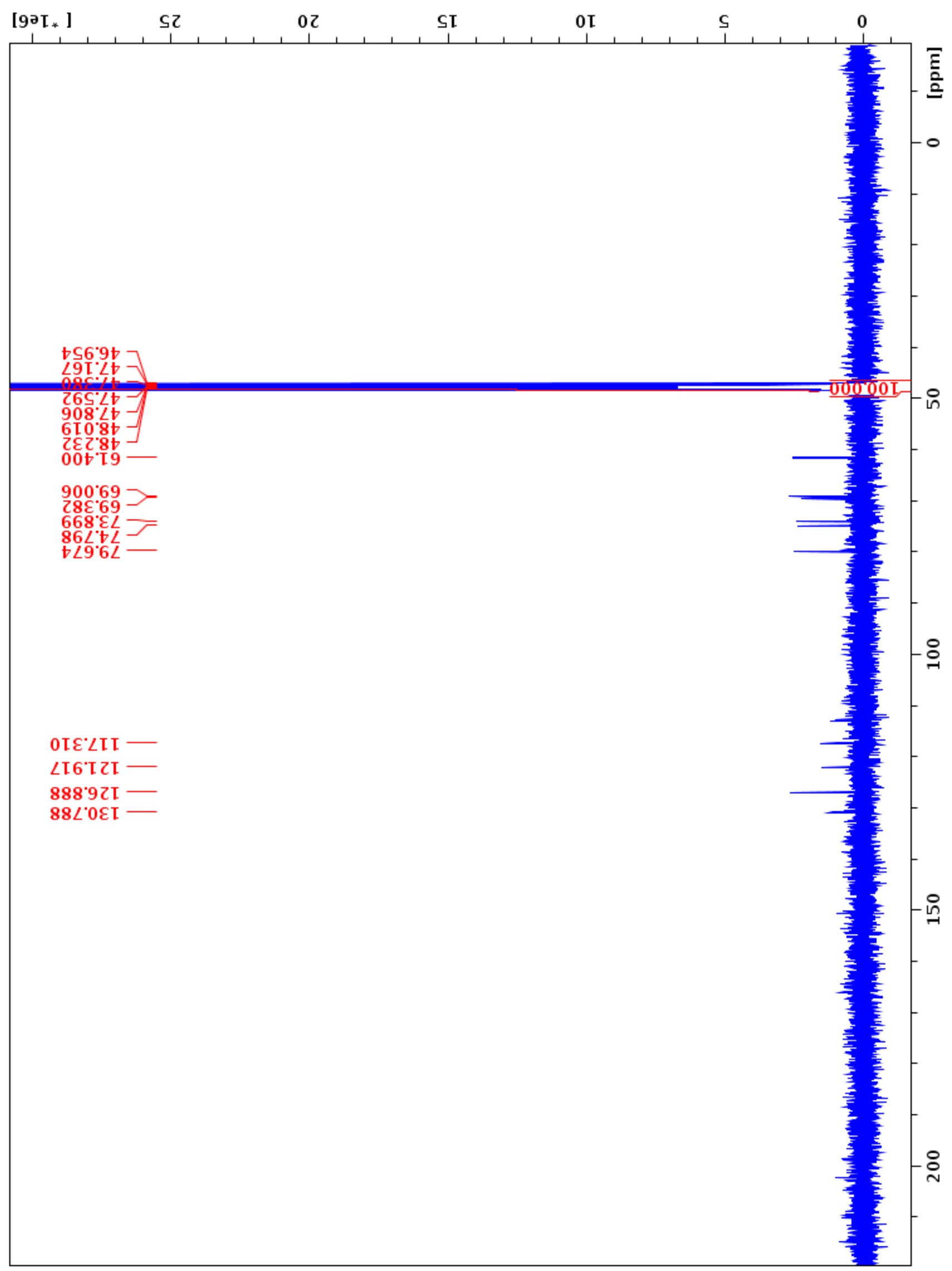


3a: 3-phenyl-5-(1-deoxy- $\beta$-D-galactopyranosyl)isoxazole

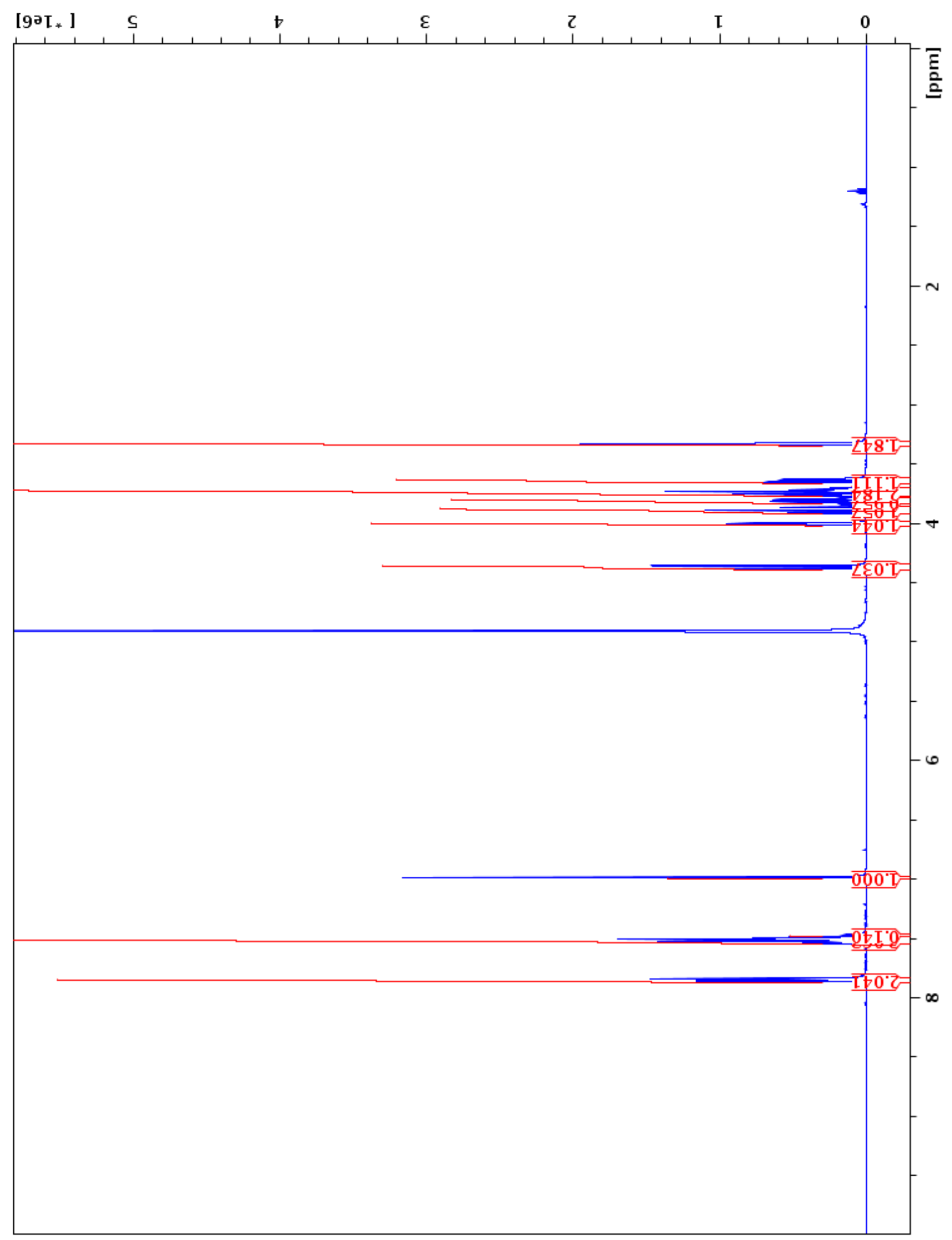


3a: 3-phenyl-5-(1-deoxy- $\beta$-D-galactopyranosyl)isoxazole

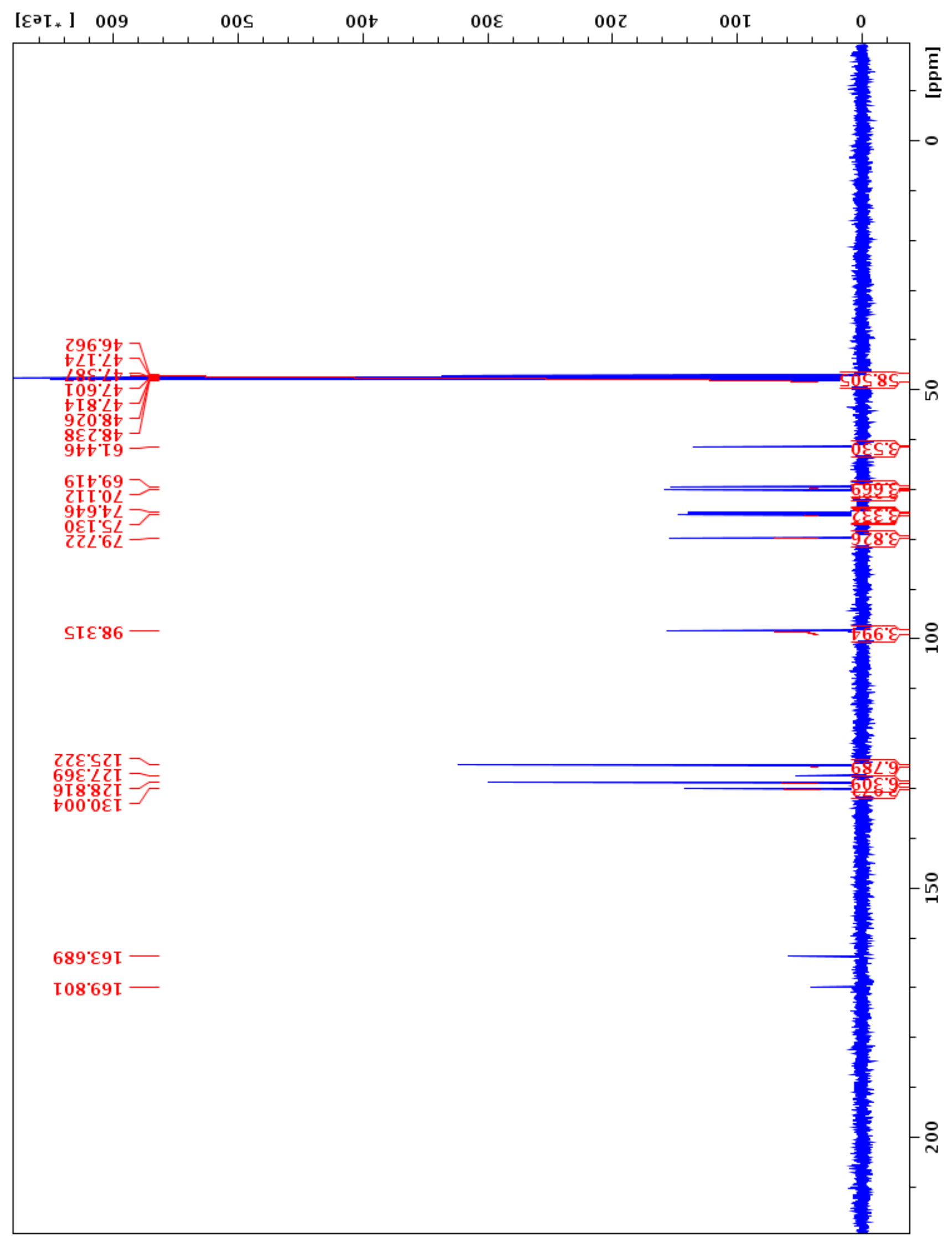


3b: 3-naphth-1-yl-(1-deoxy- $\beta$-D-galactopyranosyl)isoxazole

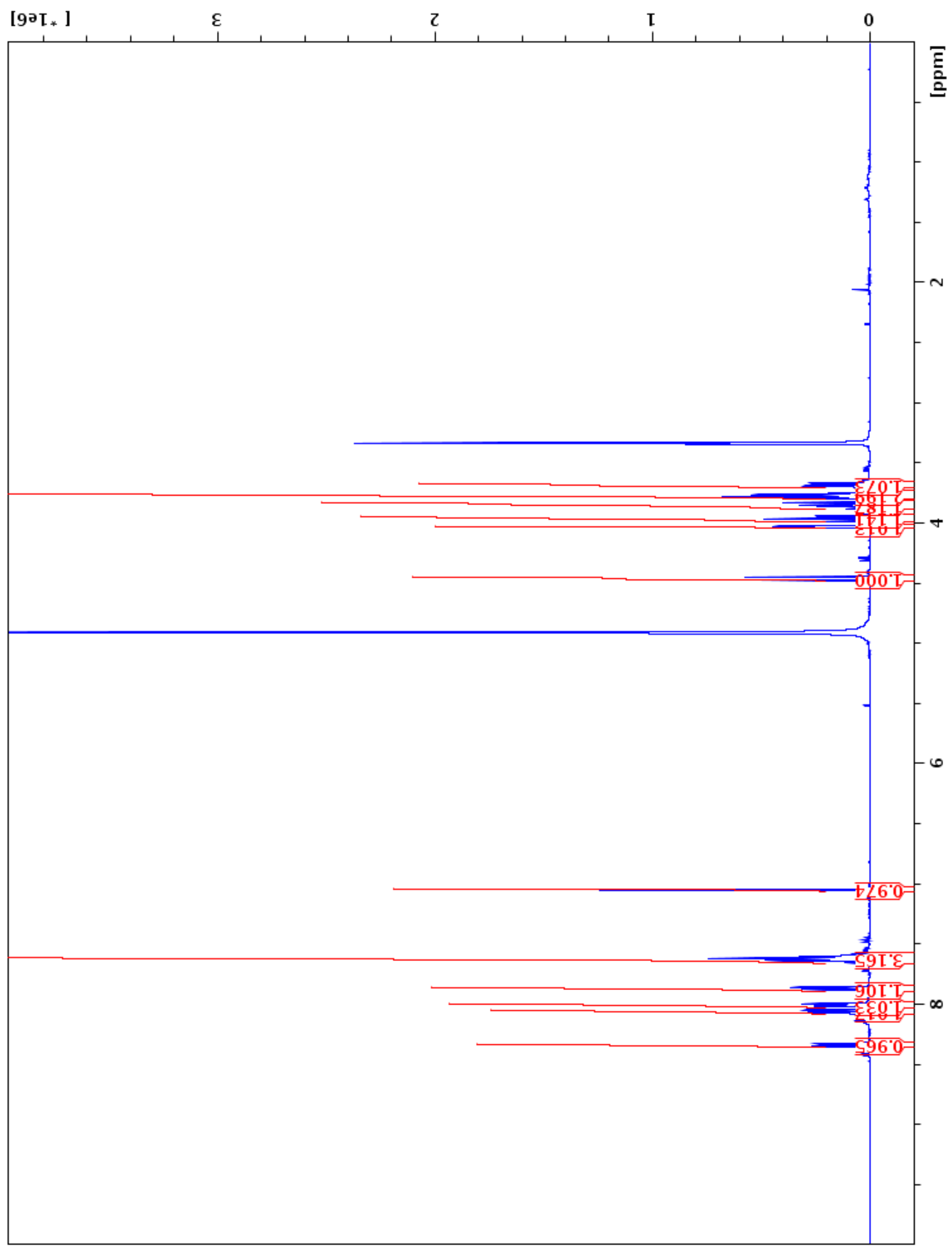


3b: 3-naphth-1-yl-5-(1-deoxy- $\beta$-D-galactopyranosyl)isoxazole

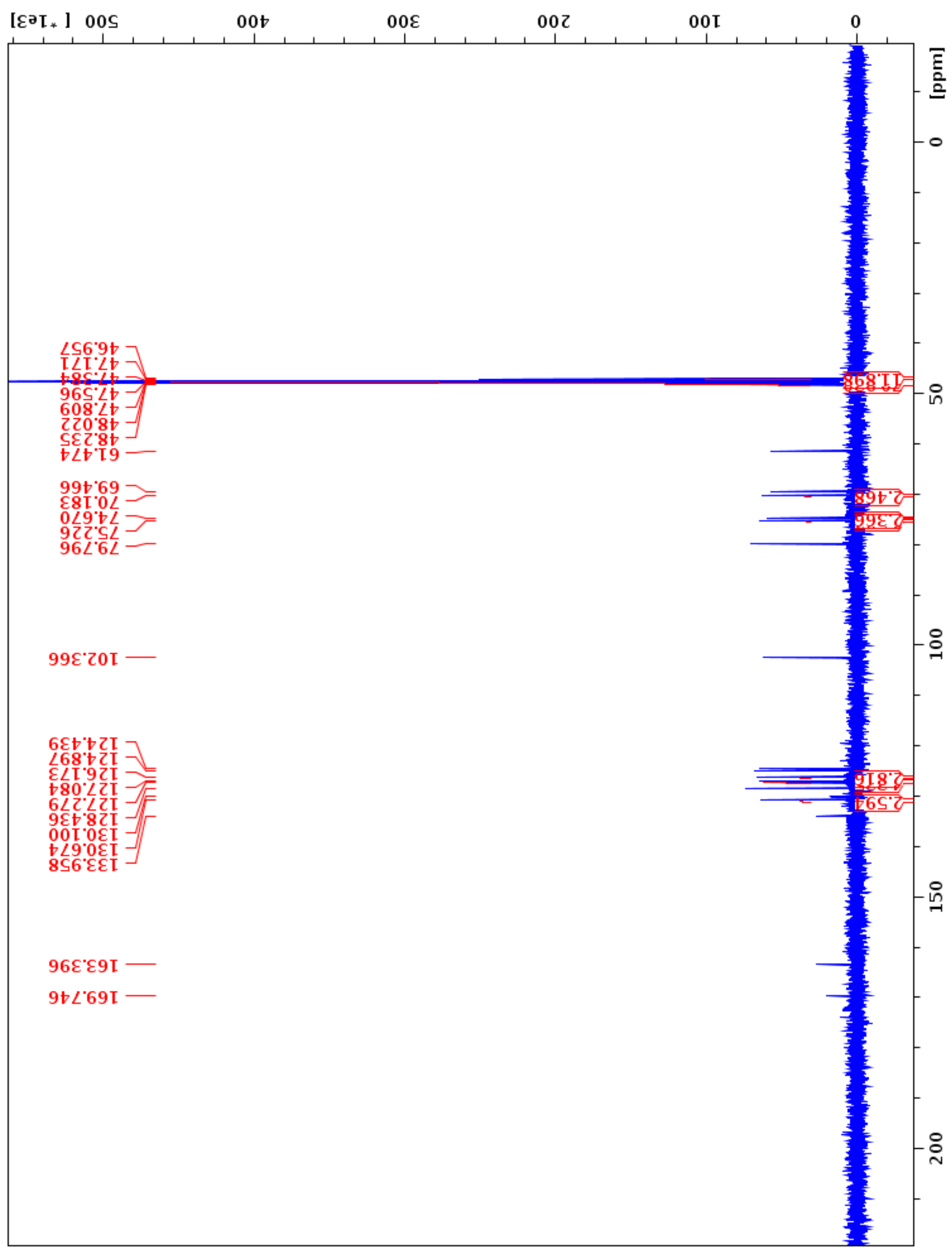


3c: 3-naphth-2-yl-5-(1-deoxy- $\beta$-D-galactopyranosyl)isoxazole

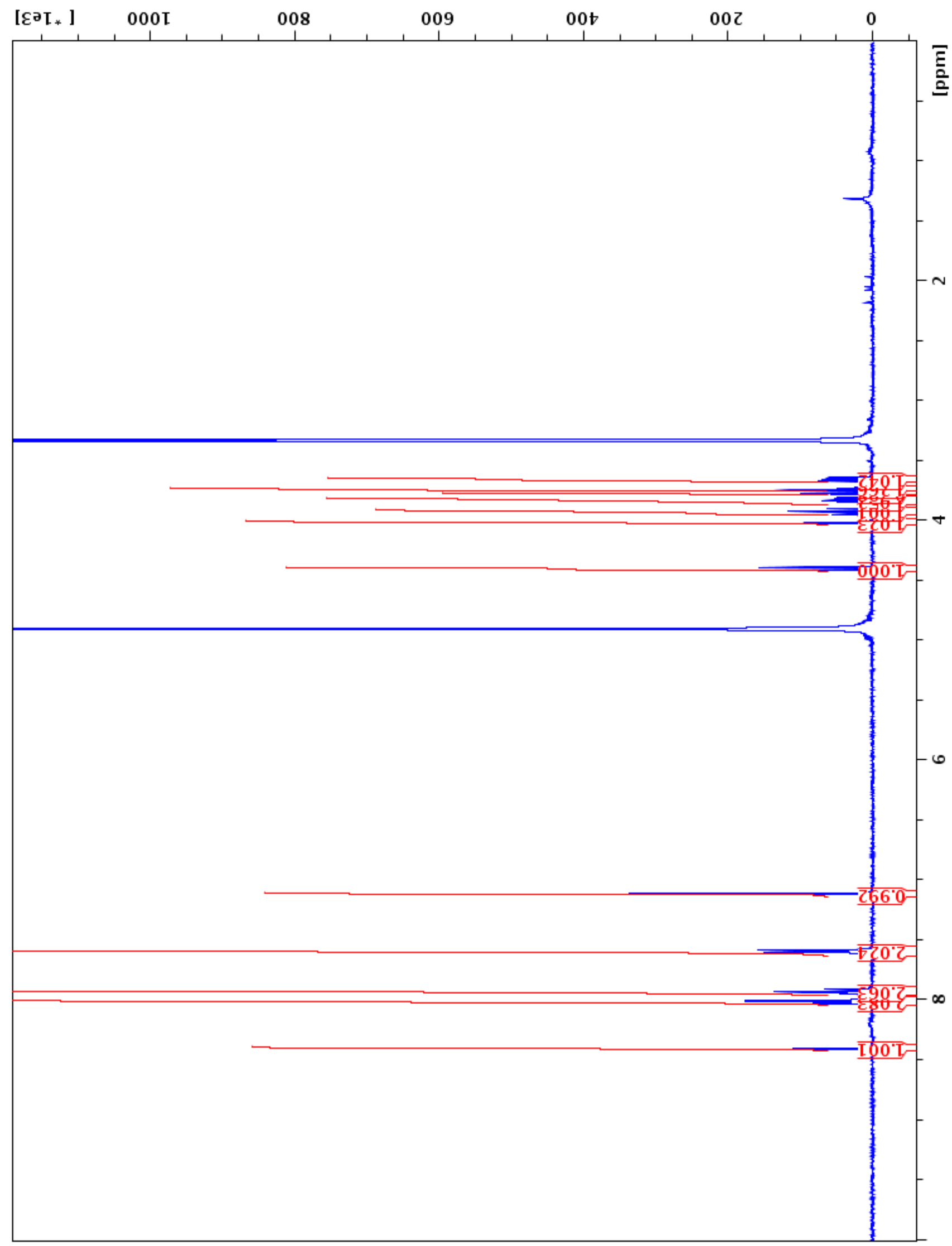


3c: 3-naphth-2-yl-5-(1-deoxy- $\beta$-D-galactopyranosyl)isoxazole

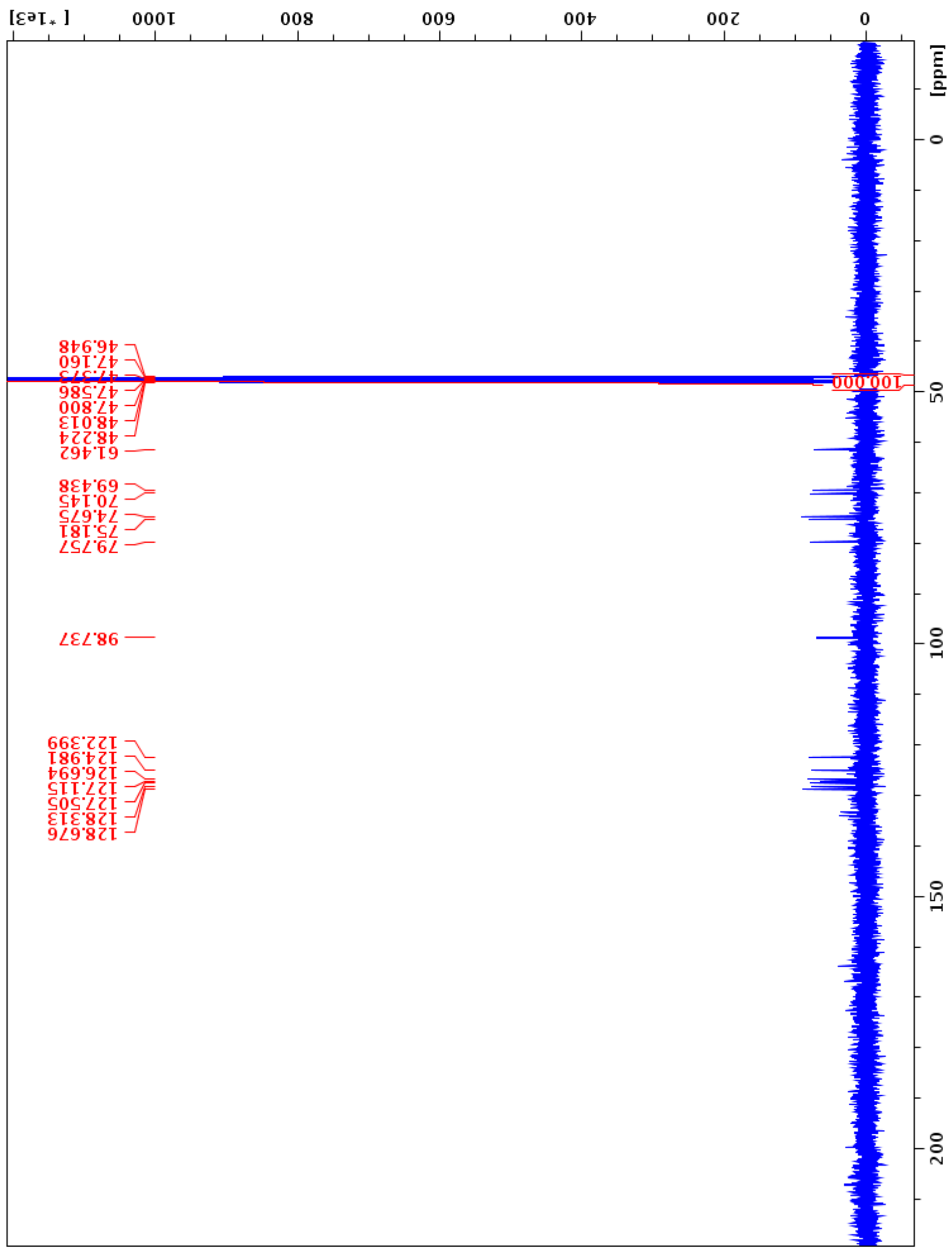


4a: 3-phenyl-5-(1-deoxy- $\beta$-D-galactopyranosyl)-1H-pyrazole

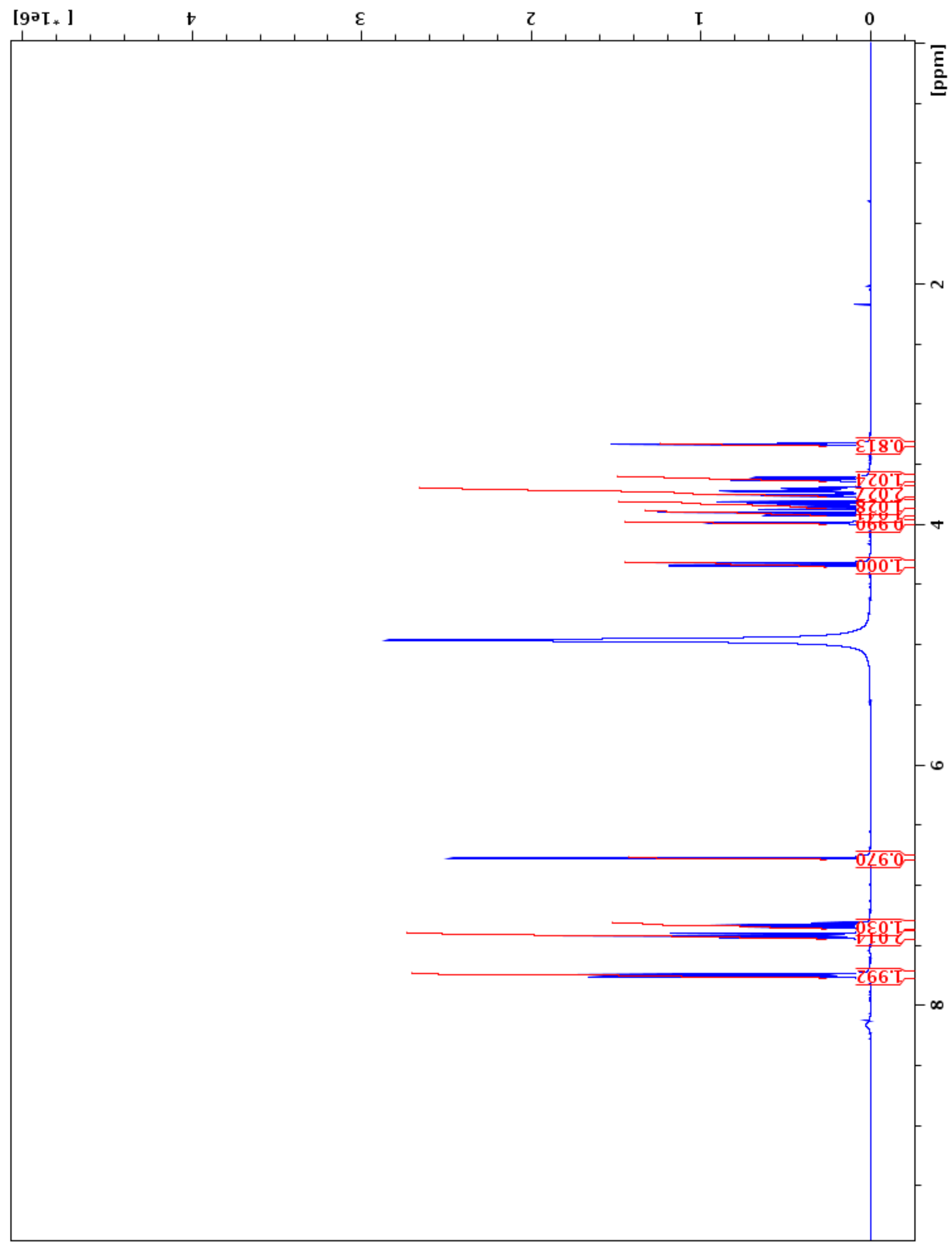


4a: 3-phenyl-5-(1-deoxy- $\beta$-D-galactopyranosyl)-1H-pyrazole

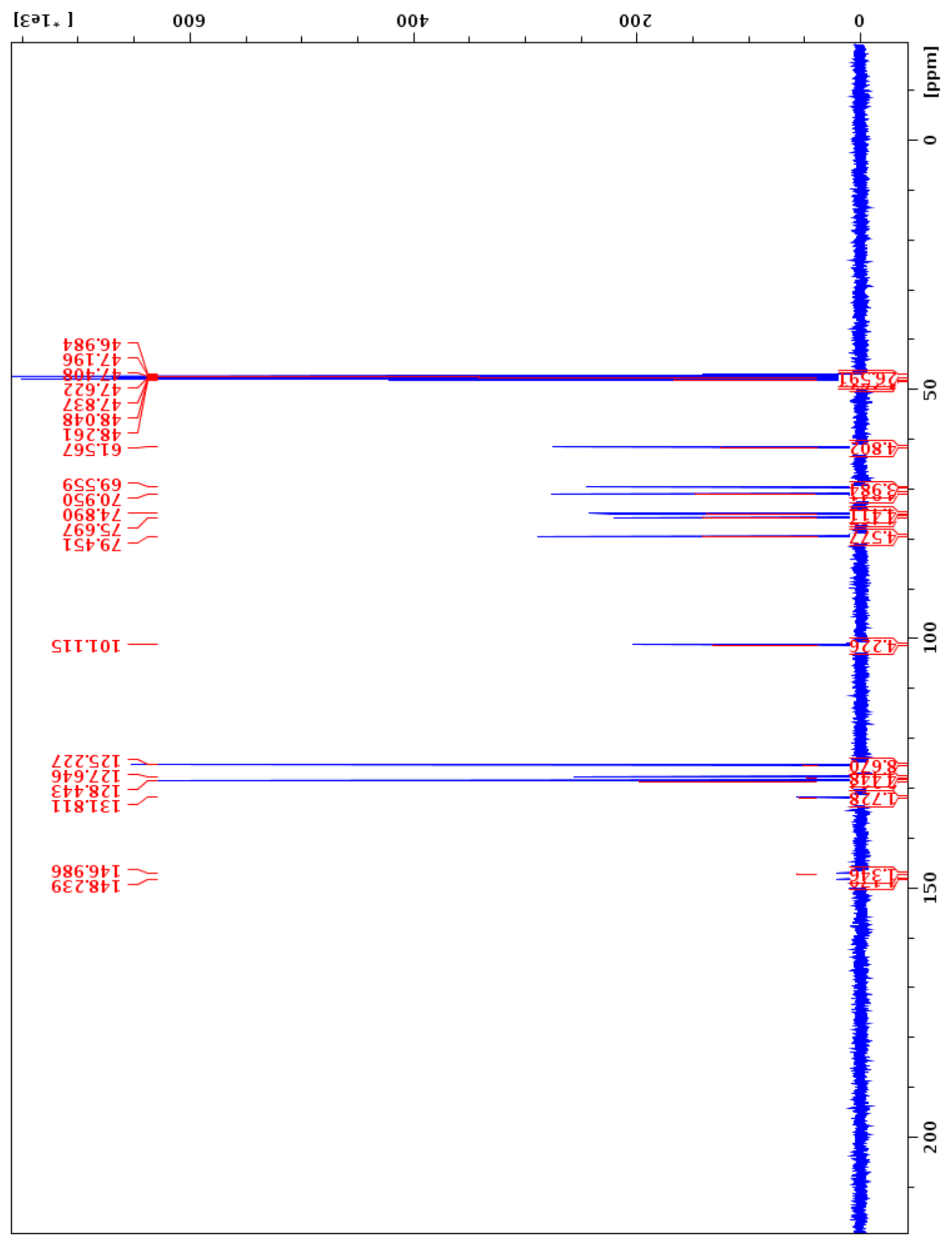


4b: 3-naphth-1-yl-5-(1-deoxy- $\beta$-D-galactopyranosyl)-1H-pyrazole

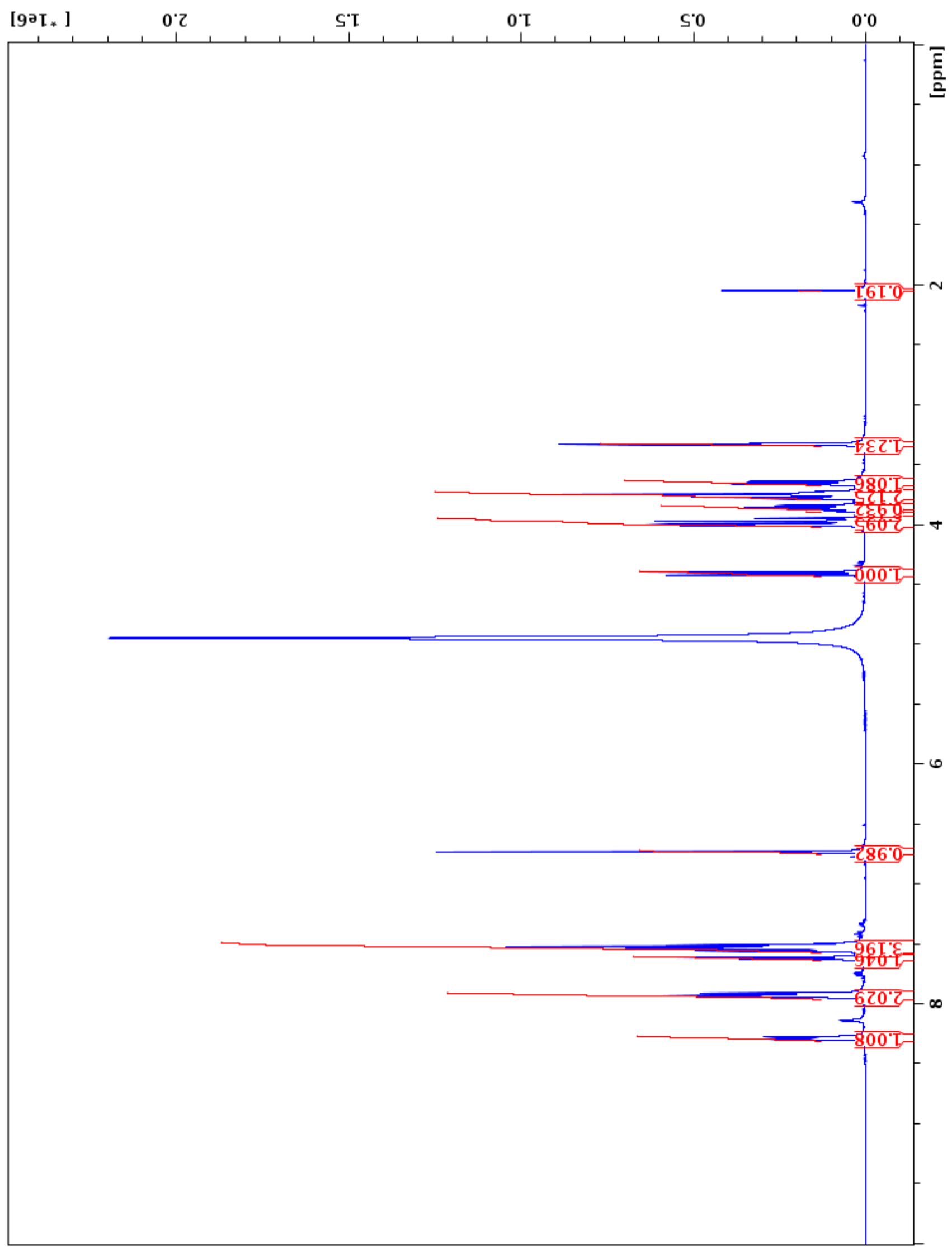


4b: 3-naphth-1-yl-5-(1-deoxy- $\beta$-D-galactopyranosyl)-1H-pyrazole

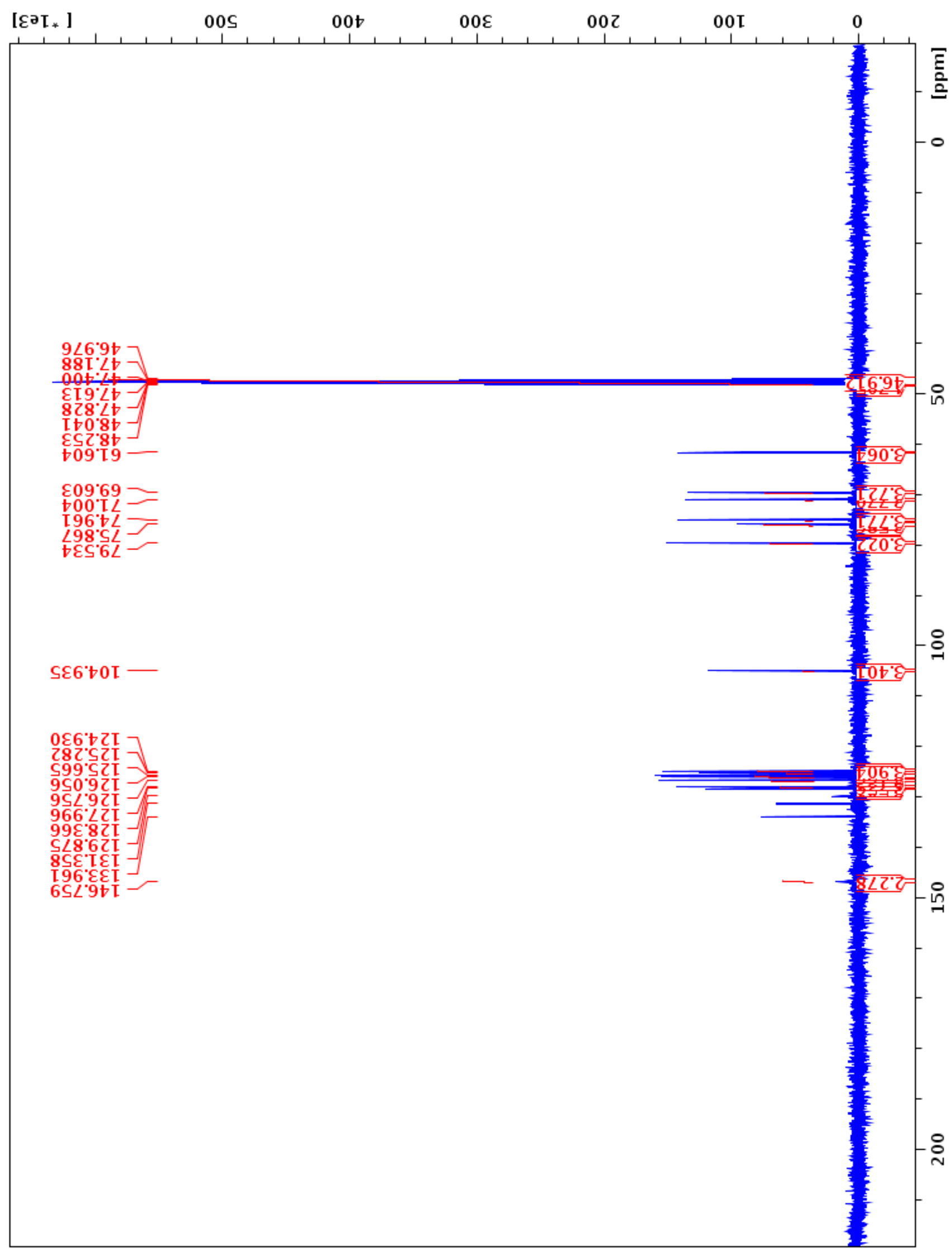


4c: 3-naphth-2-yl-5-(1-deoxy- $\beta$-D-galactopyranosyl)-1H-pyrazole

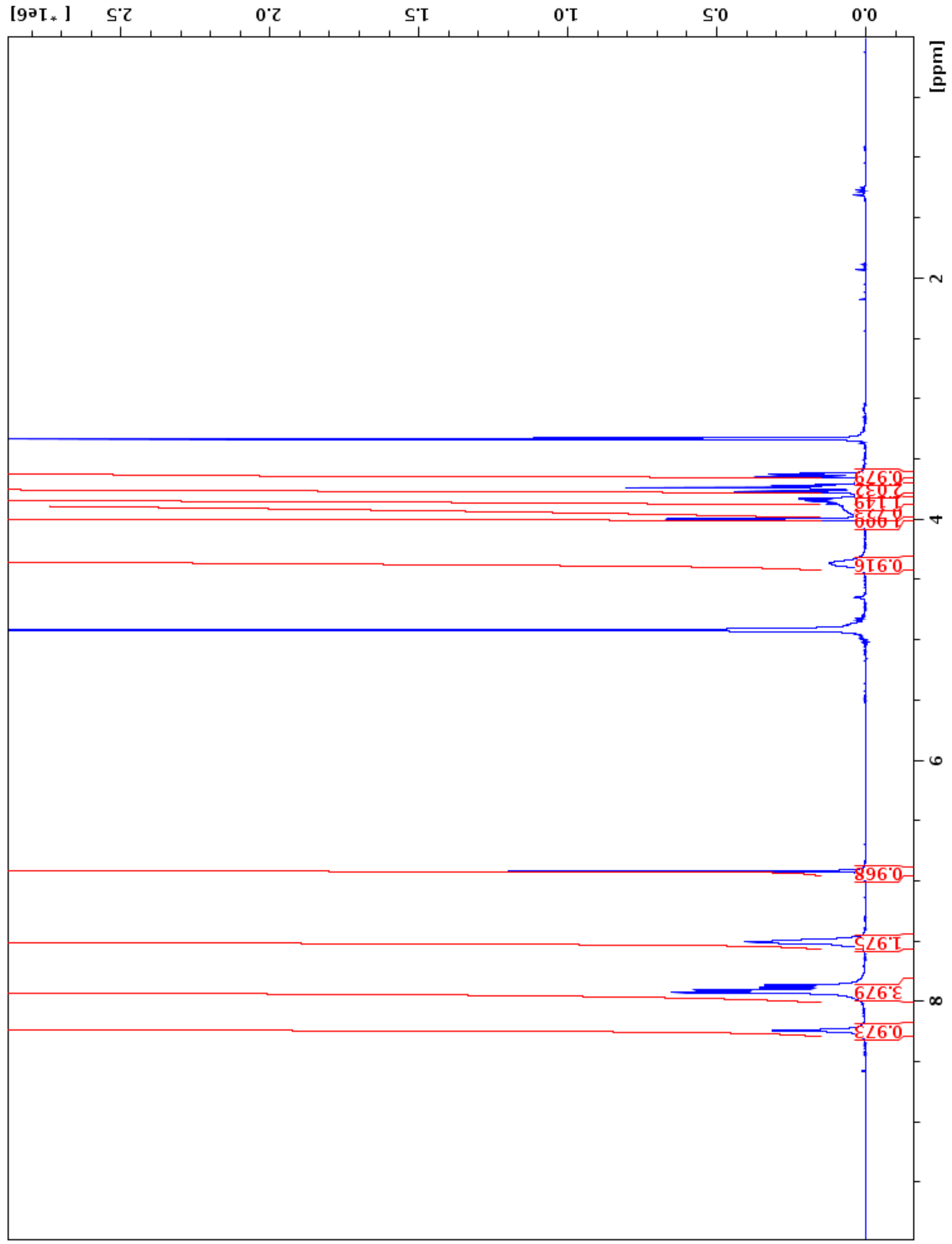


4c: 3-naphth-2-yl-5-(1-deoxy- $\beta$-D-galactopyranosyl)-1H-pyrazole

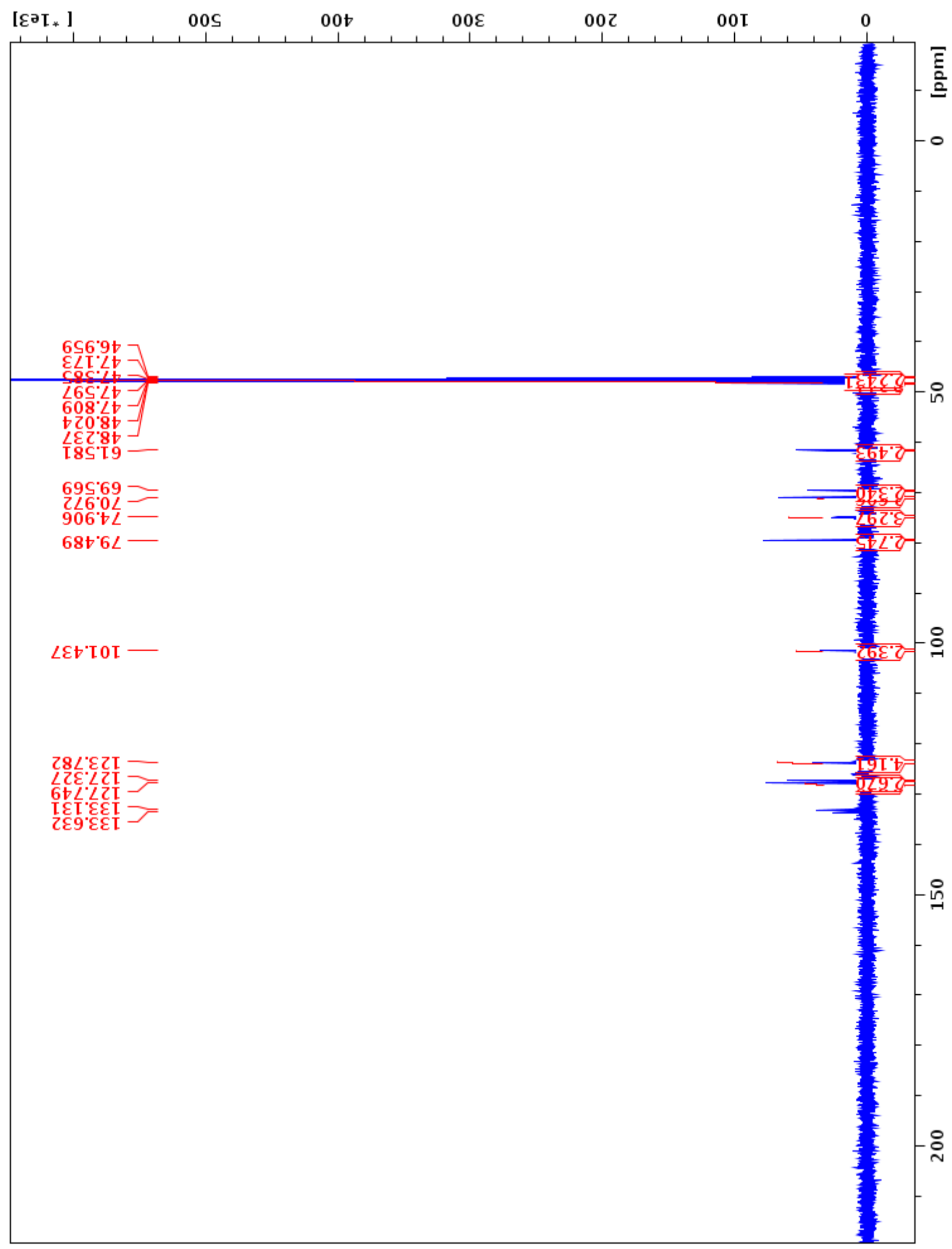


9a: 1 -phenyl-3-(2,3,4,6-tetra- $O$-acetyl- $\beta$-D-galactopyranosyl)-prop-2-yn-1-one

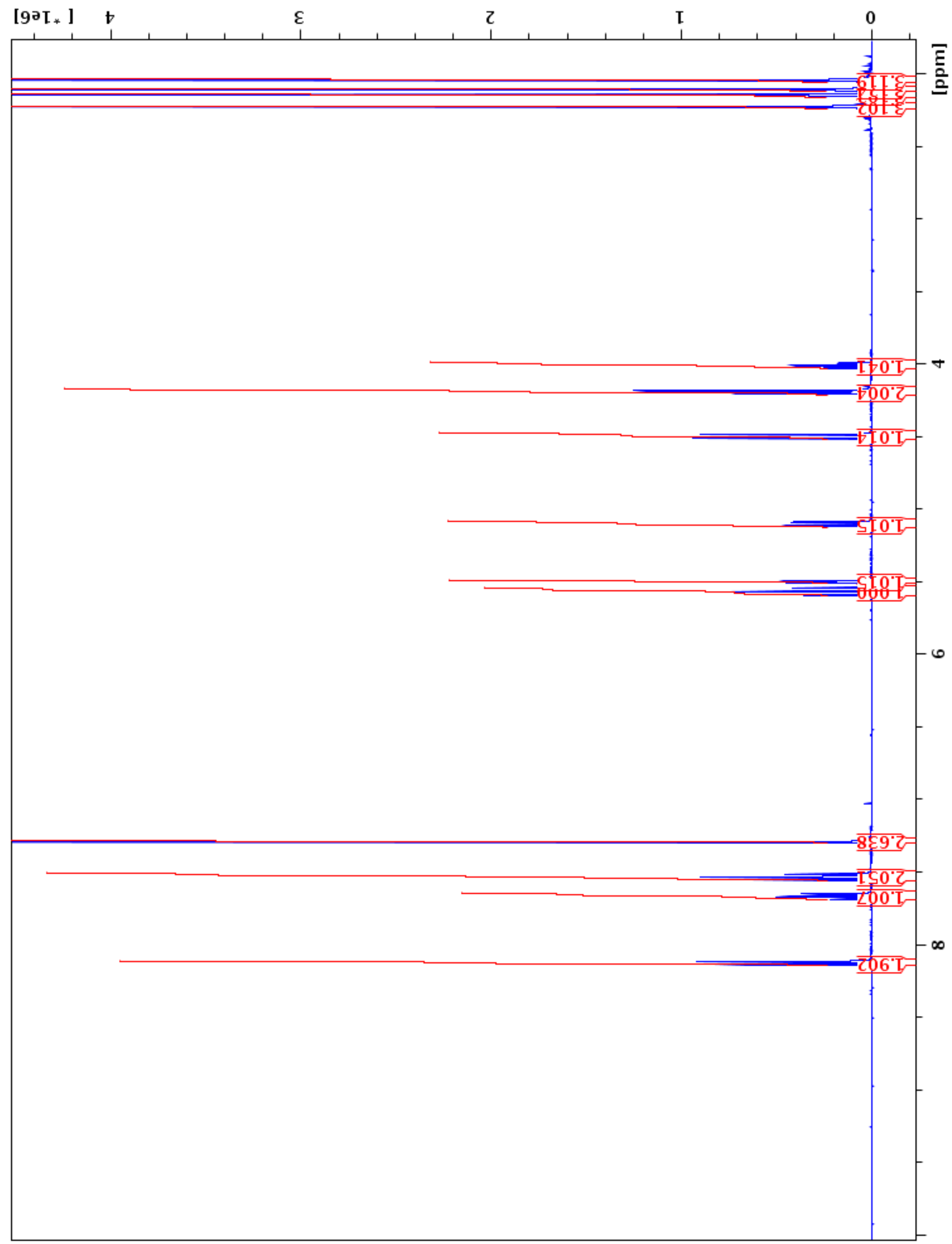


9a: 1-phenyl-3-(2,3,4,6-tetra- $O$-acetyl- $\beta$-D-galactopyranosyl)-prop-2-yn-1-one

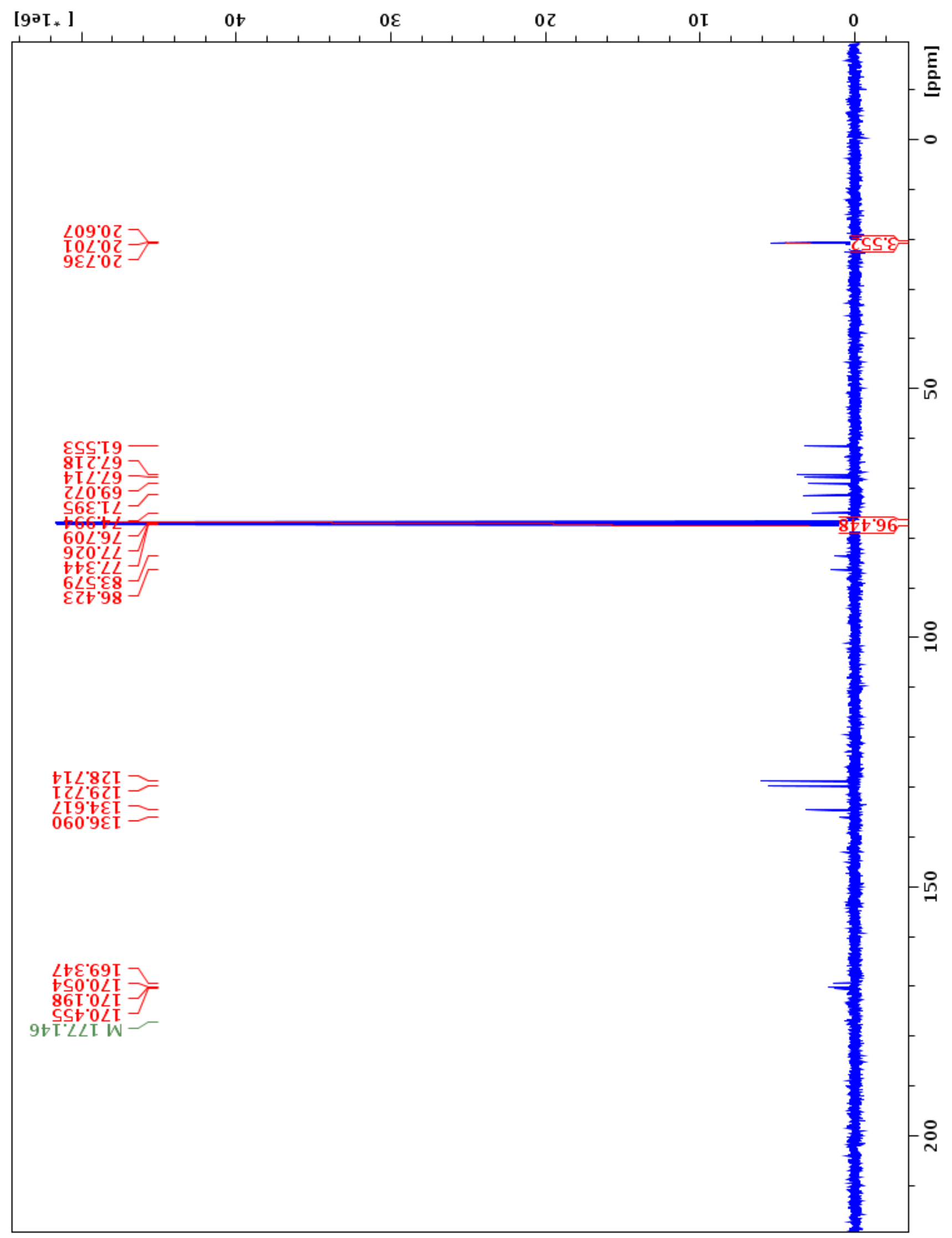


9b: 1-naphth-2-yl-3-(2,3,4,6-tetra- $O$-acetyl- $\beta$-D-galactopyranosyl)-prop-2-yn-1one

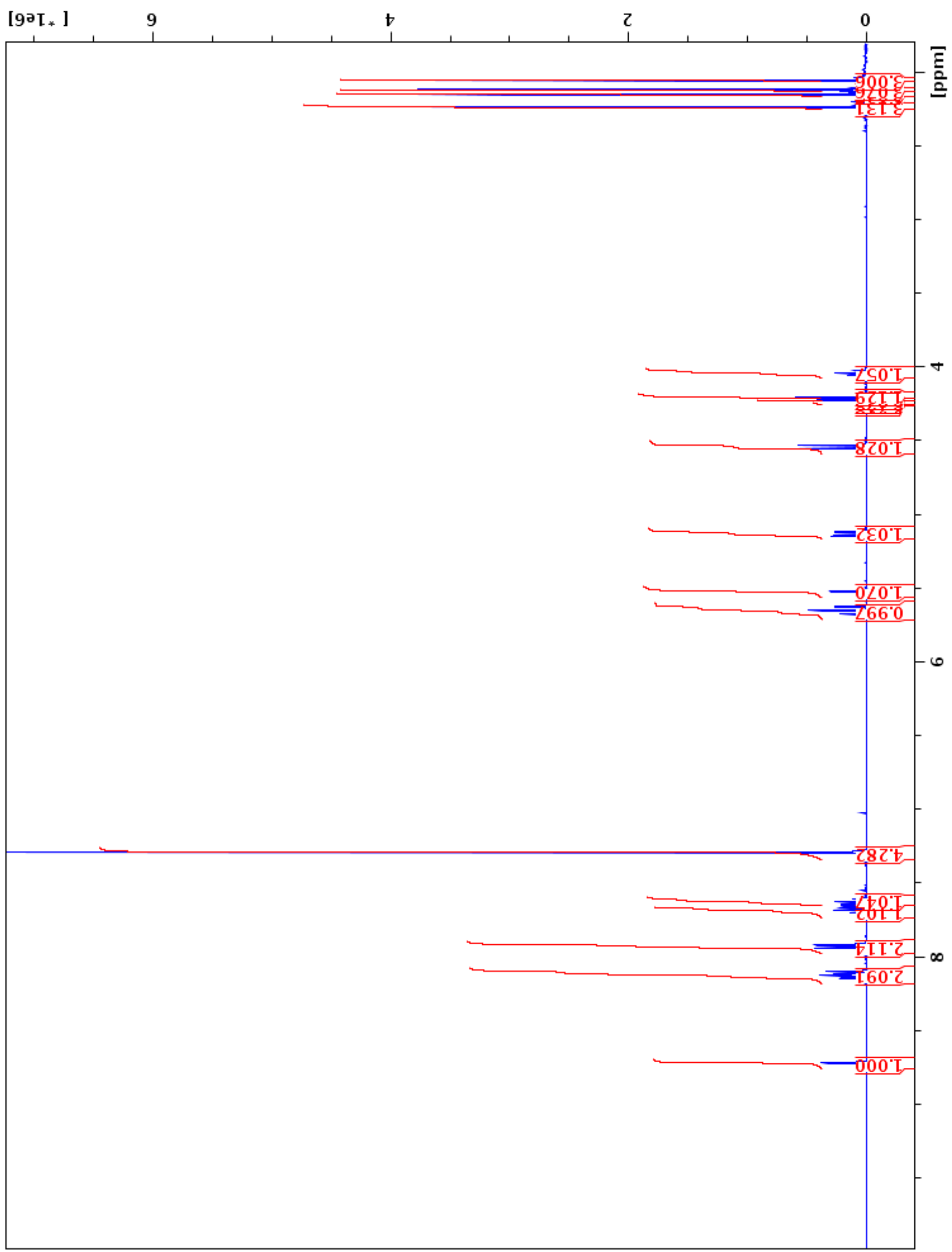


9b: 1-naphth-2-yl-3-(2,3,4,6-tetra- $O$-acetyl- $\beta$-D-galactopyranosyl)-prop-2-yn-1one

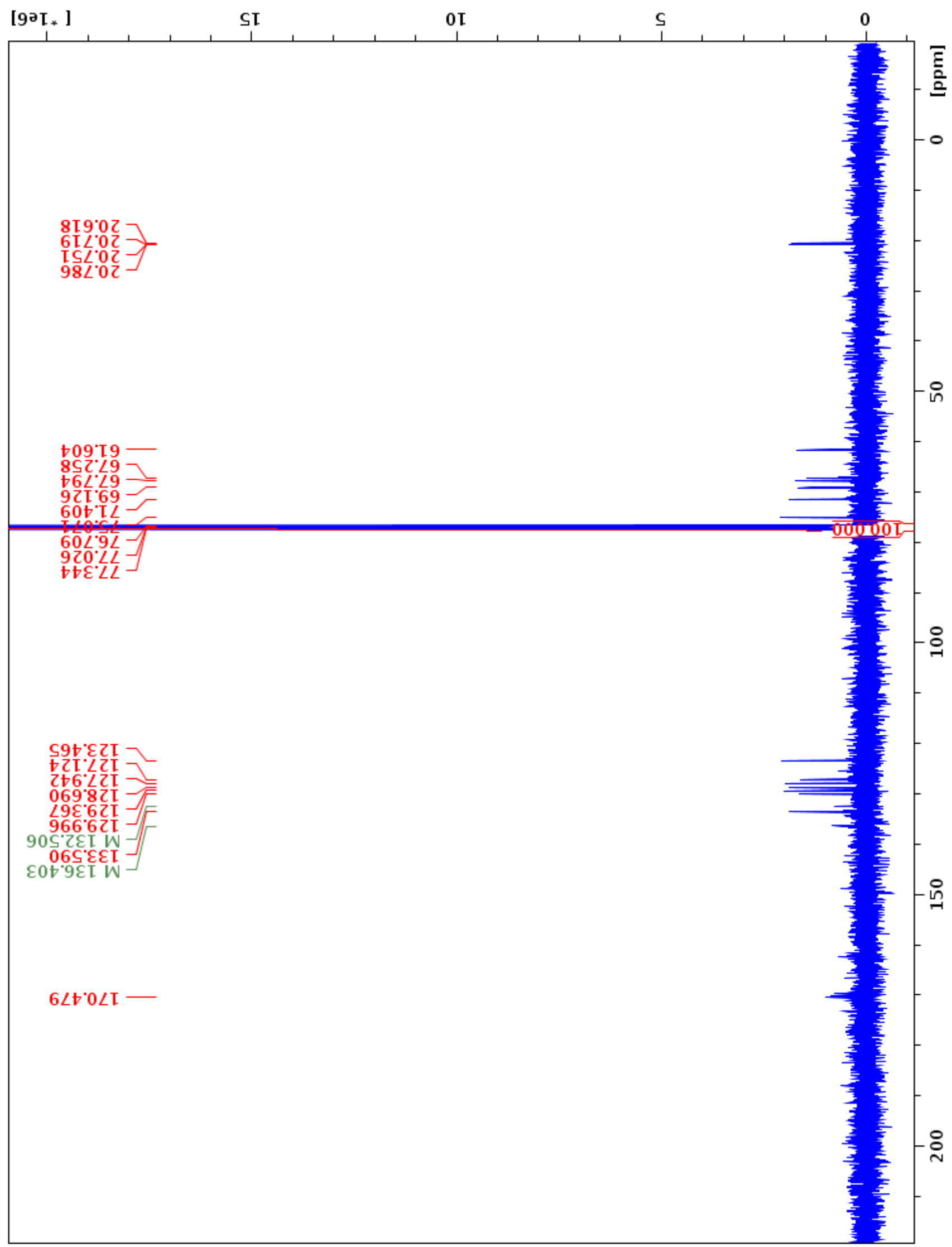


9c: 1 -naphth-1-yl-3-(2,3,4,6-tetra- $O$-acetyl- $\beta$-D-galactopyranosyl)-prop-2-yn-1one

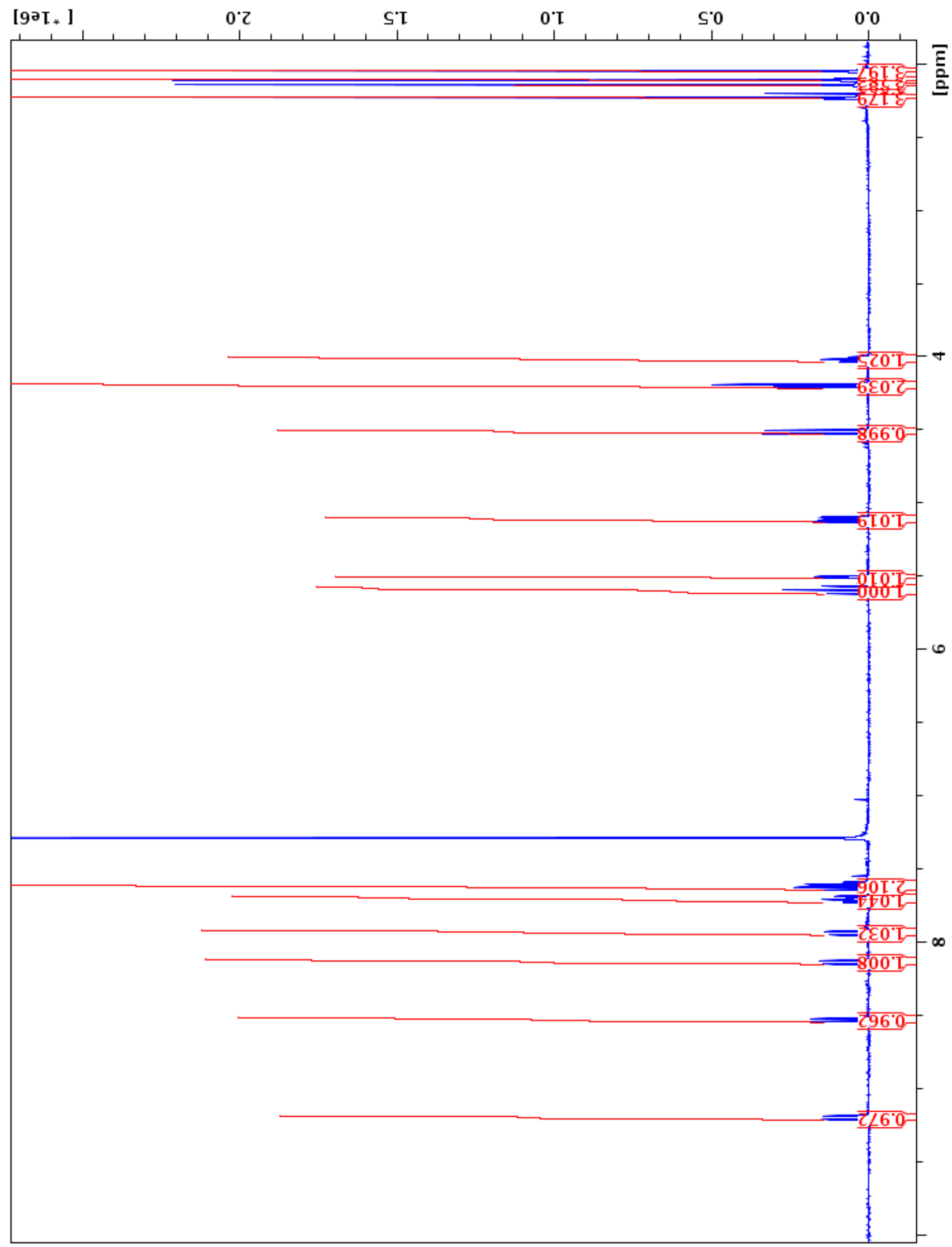


9c: 1 -naphth-1-yl-3-(2,3,4,6-tetra- 0 -acetyl- $\beta$-D-galactopyranosyl)-prop-2-yn-1one

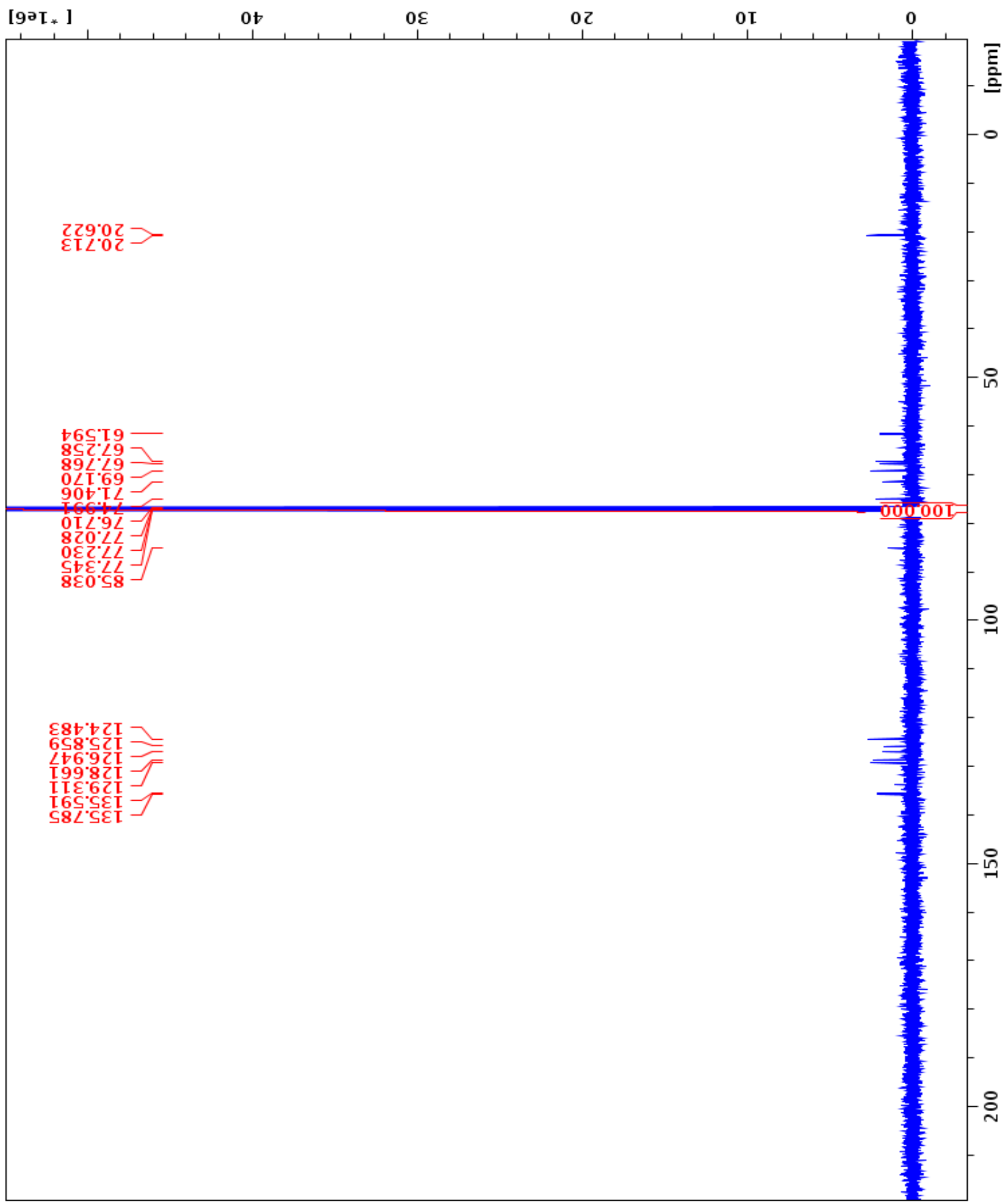


10a: 2-phenyl-5-(2,3,4,6-tetra-O-acetyl-1-deoxy- $\beta$-D-galactopyranosyl)oxazole

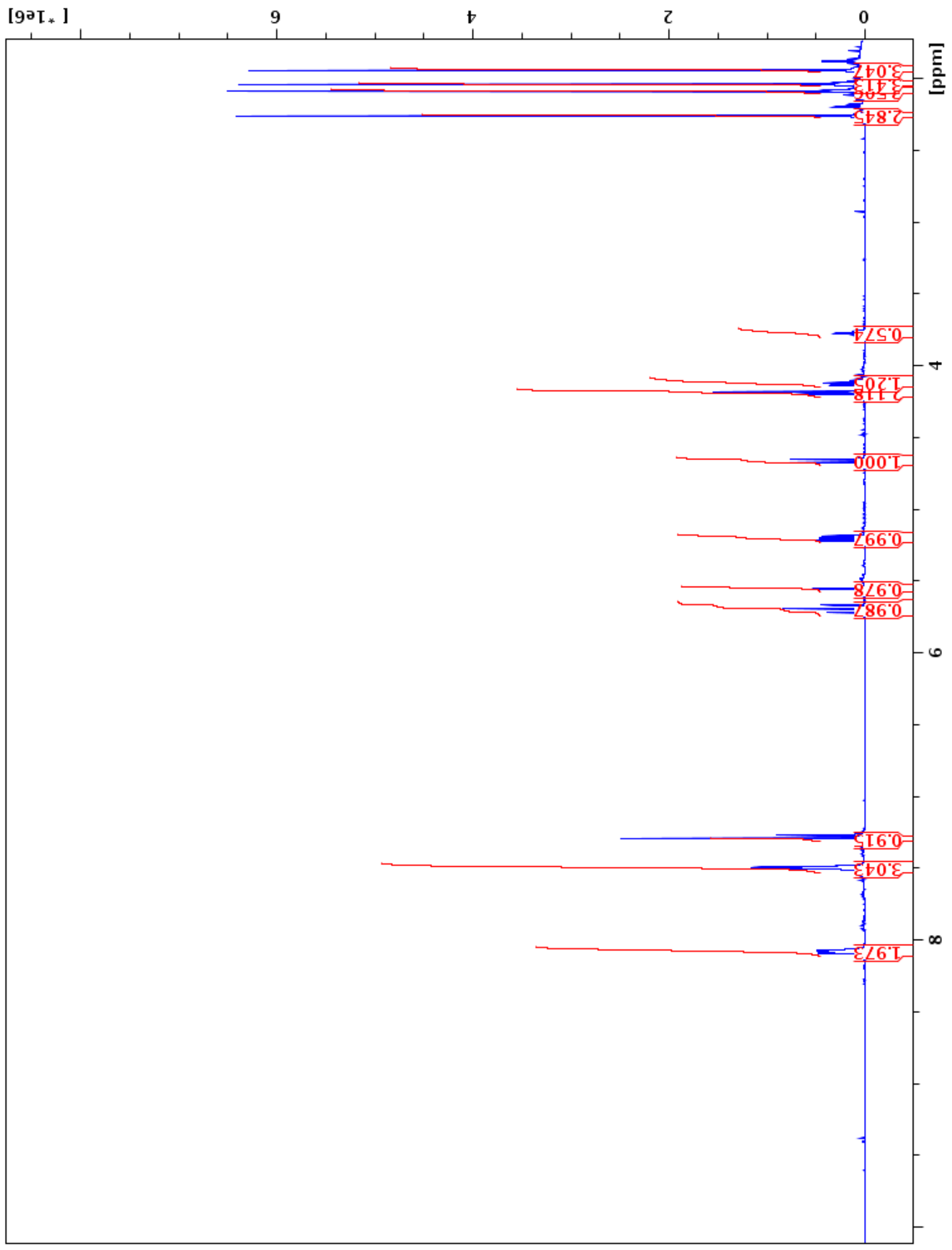


10a: 2 -phenyl-5-(2,3,4,6-tetra- $O$-acetyl-1-deoxy- $\beta$-D-galactopyranosyl)oxazole

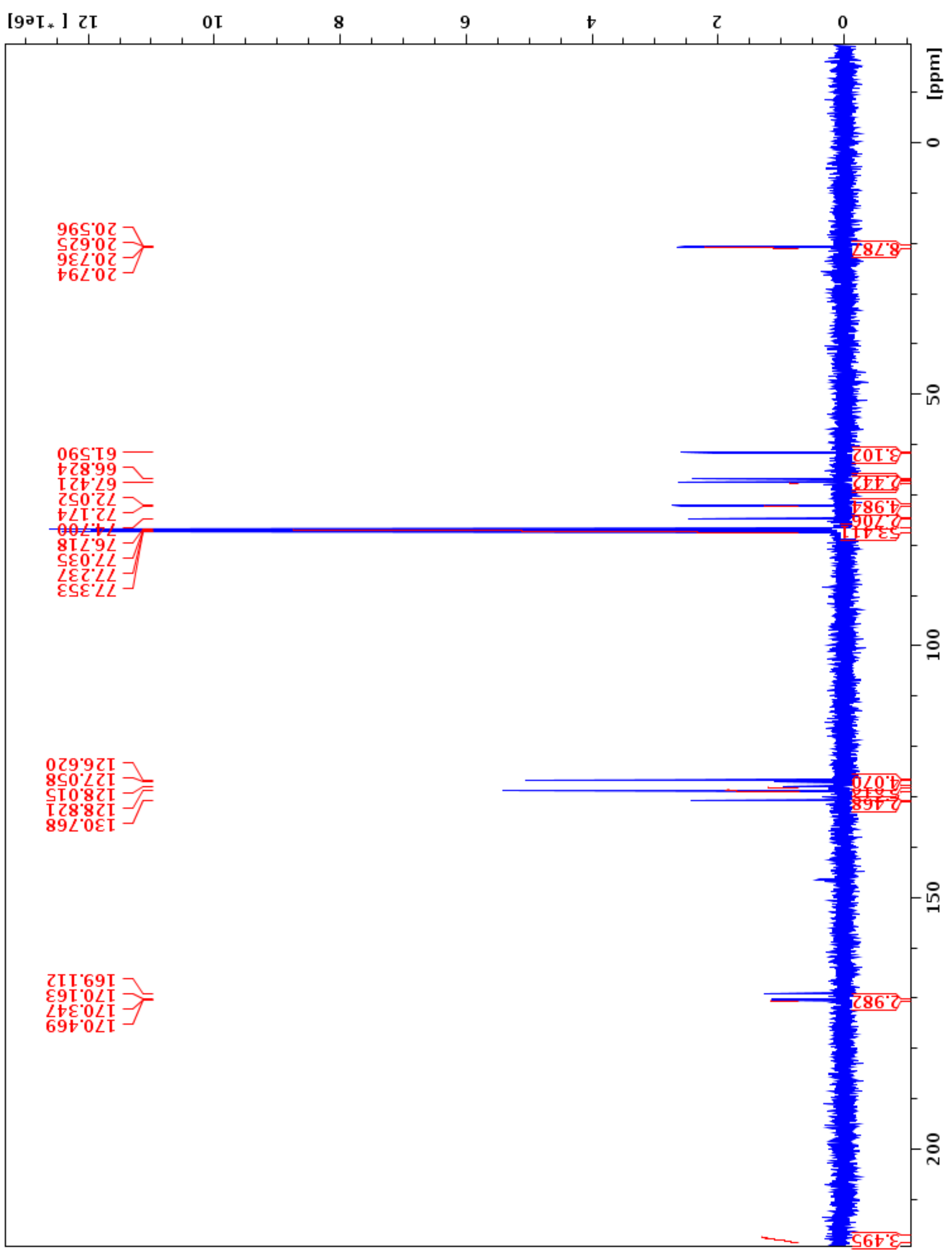


10b: 2-naphth-1-yl-5-(2,3,4,6-tetra-O-acetyl-1-deoxy- $\beta$-D-galactopyranosyl)oxazole

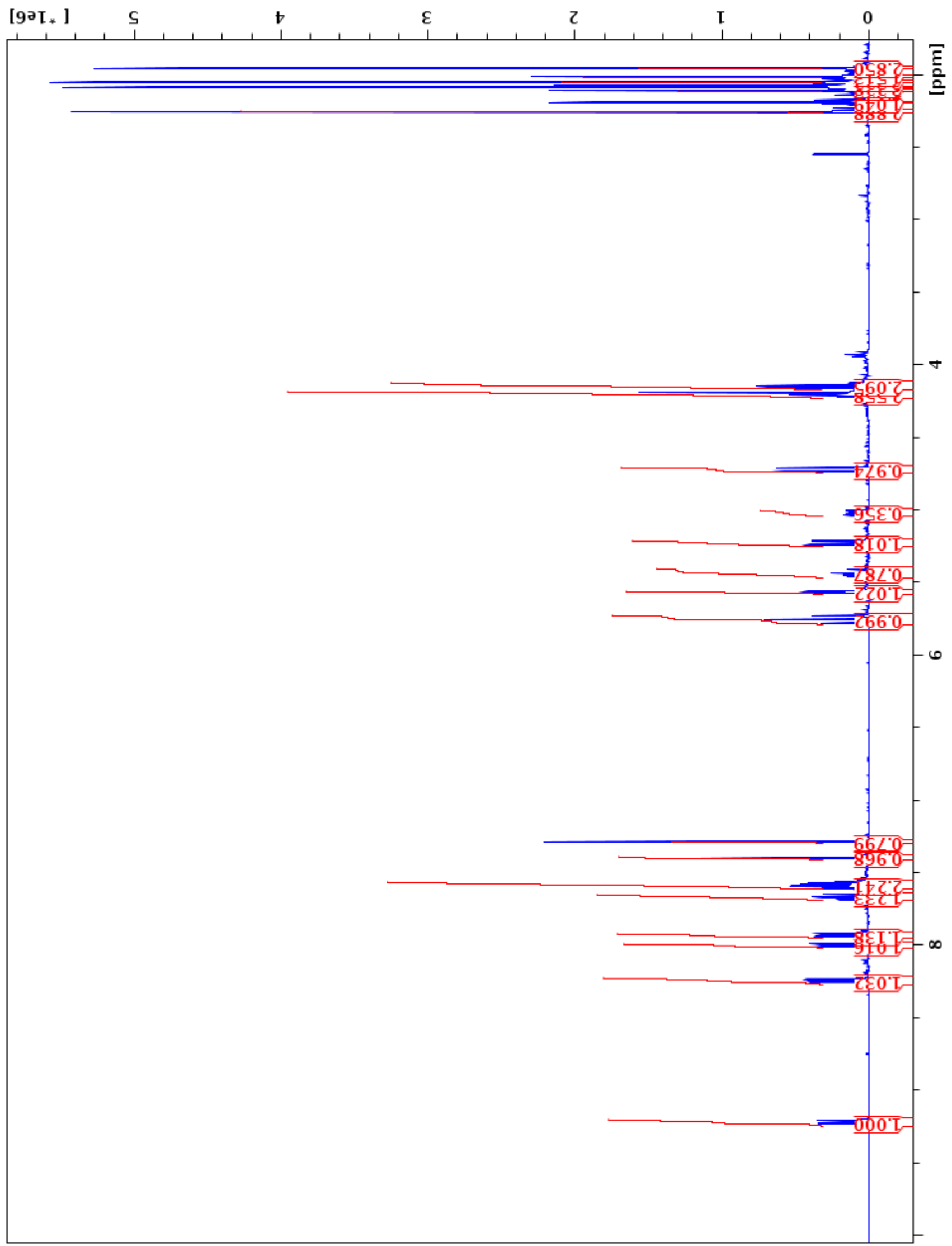


10b: 2-naphth-1-yl-5-(2,3,4,6-tetra-O-acetyl-1-deoxy- $\beta$-D-galactopyranosyl)oxazole

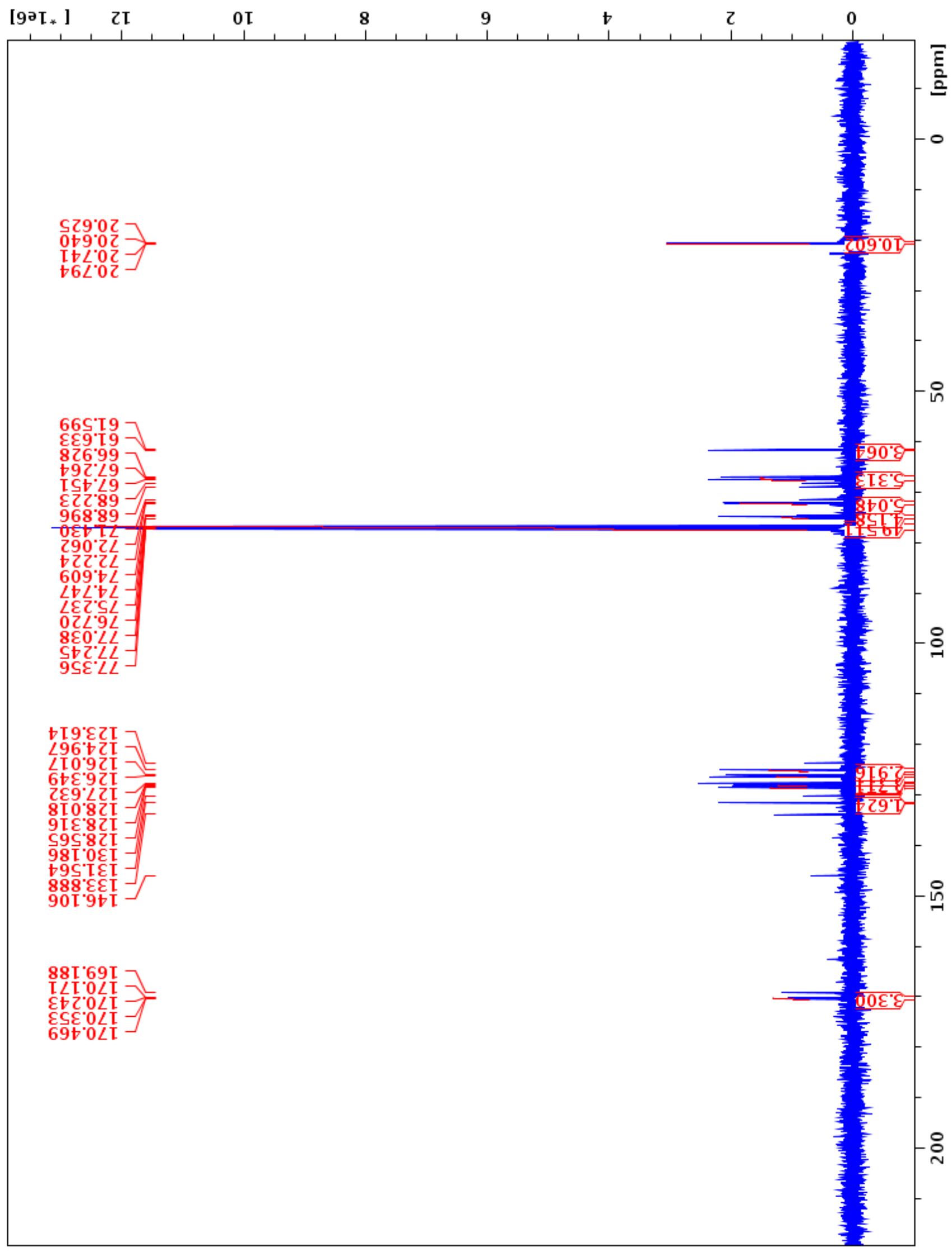


10c: 2 -naphth-2-yl-5-(2,3,4,6-tetra- $O$-acetyl-1-deoxy- $\beta$-D-galactopyranosyl)oxazole

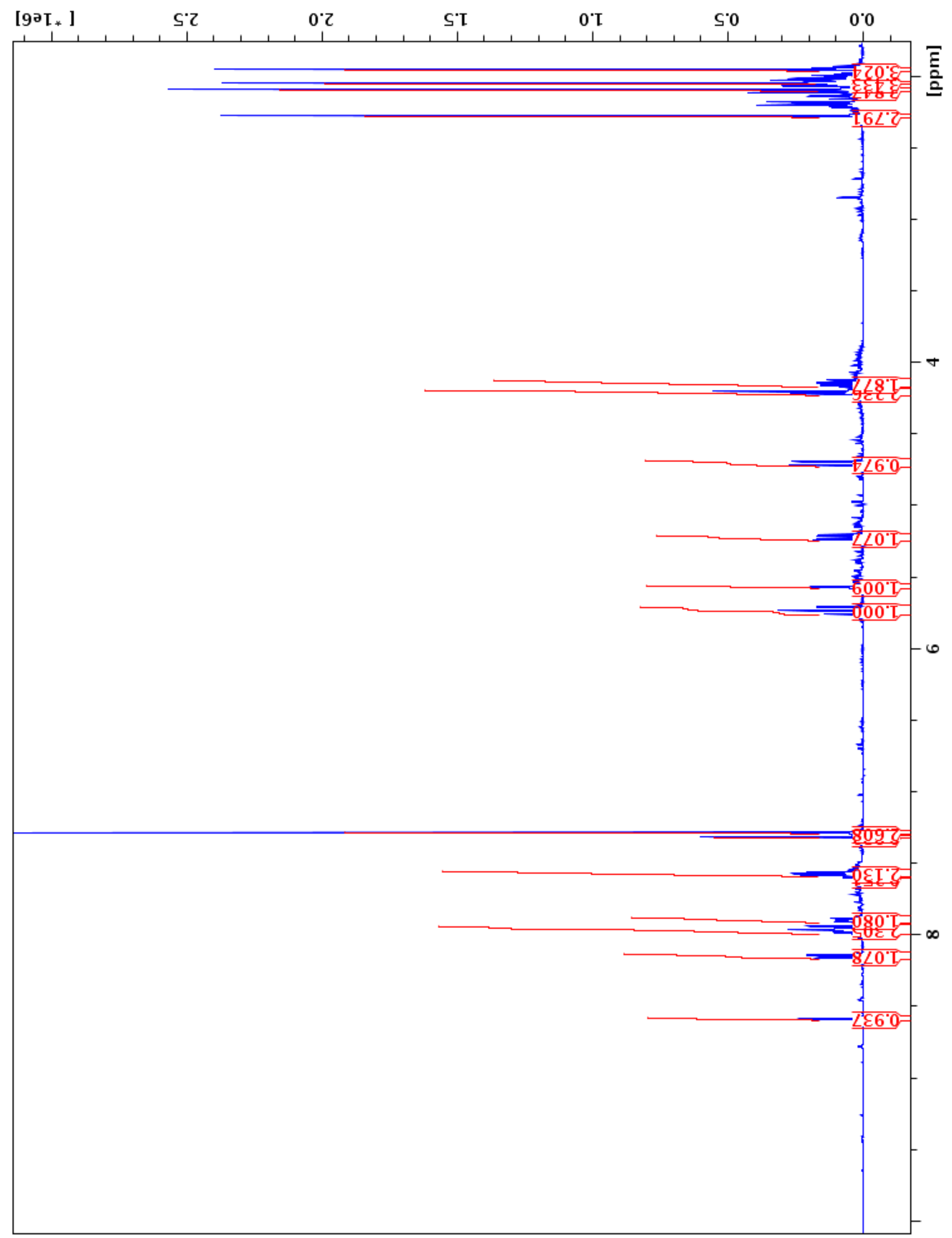


10c: 2 -naphth-2-yl-5-(2,3,4,6-tetra-O-acetyl-1-deoxy- $\beta$-D-galactopyranosyl)oxazole

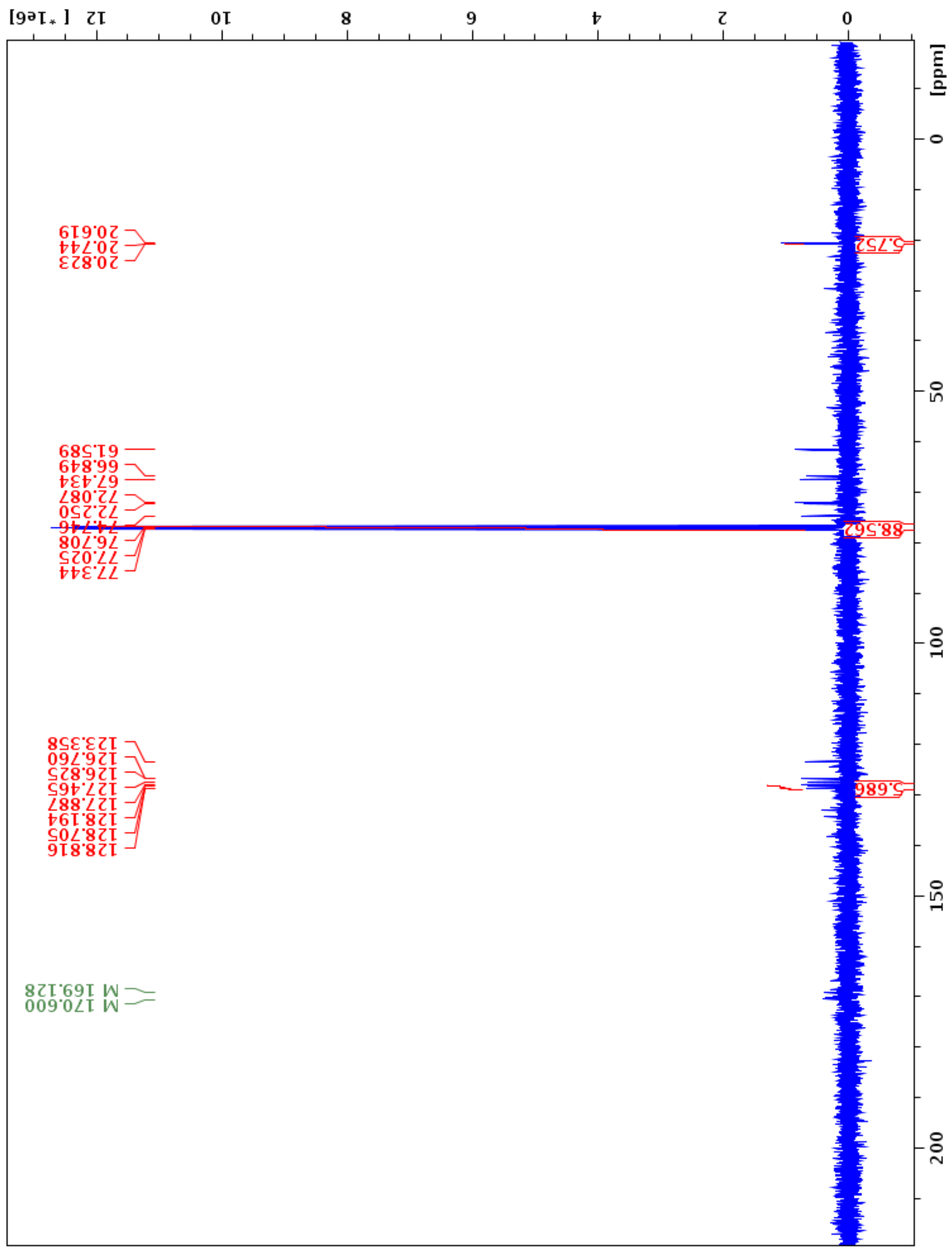


10d: 2-(4-chlorophenyl)-5-(2,3,4,6-tetra- $O$-acetyl-1-deoxy- $\beta$-D-galactopyranosyl)oxazole

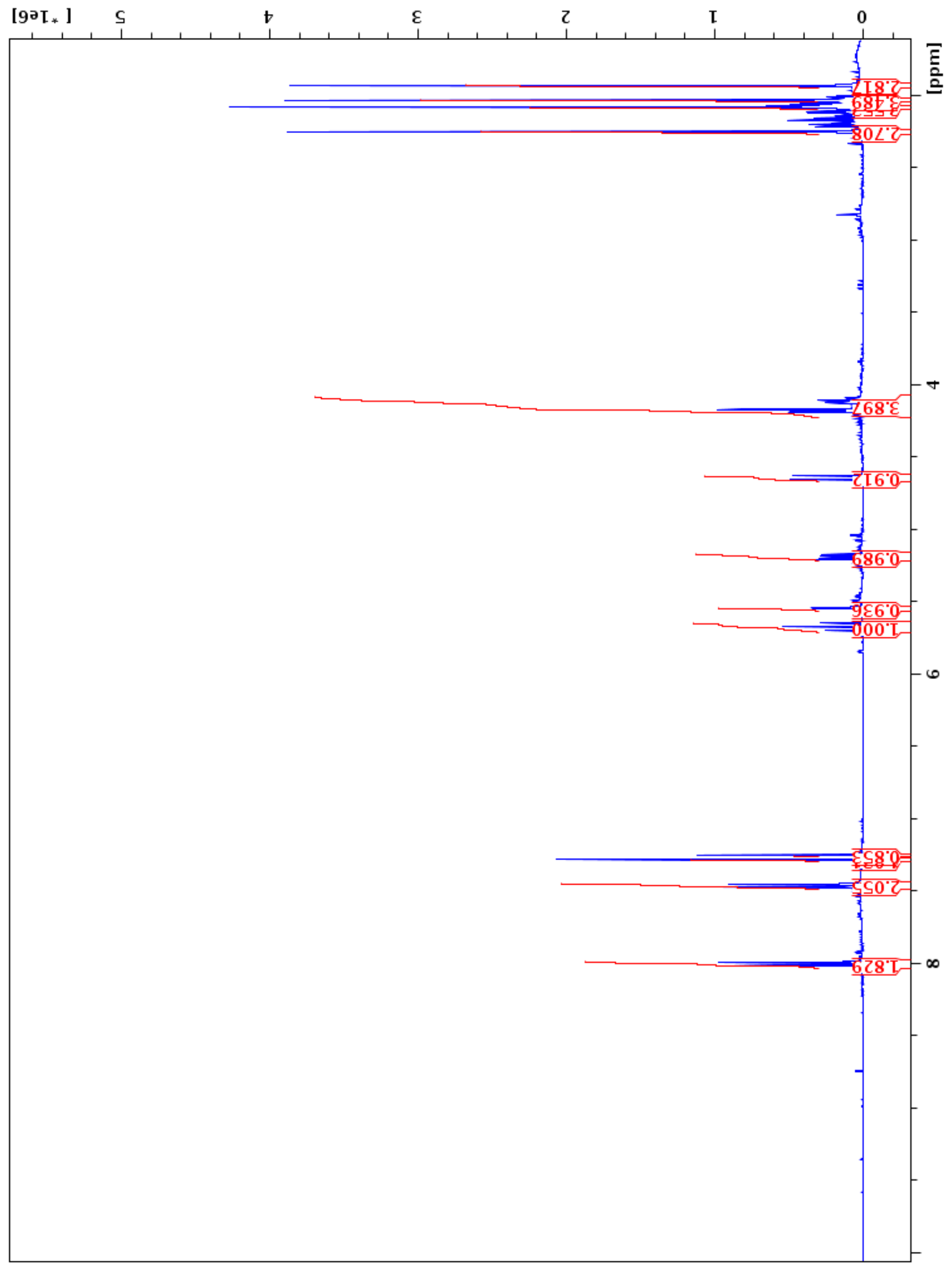


10d: 2-(4-chlorophenyl)-5-(2,3,4,6-tetra- $O$-acetyl-1-deoxy- $\beta$-D-galactopyranosyl)oxazole

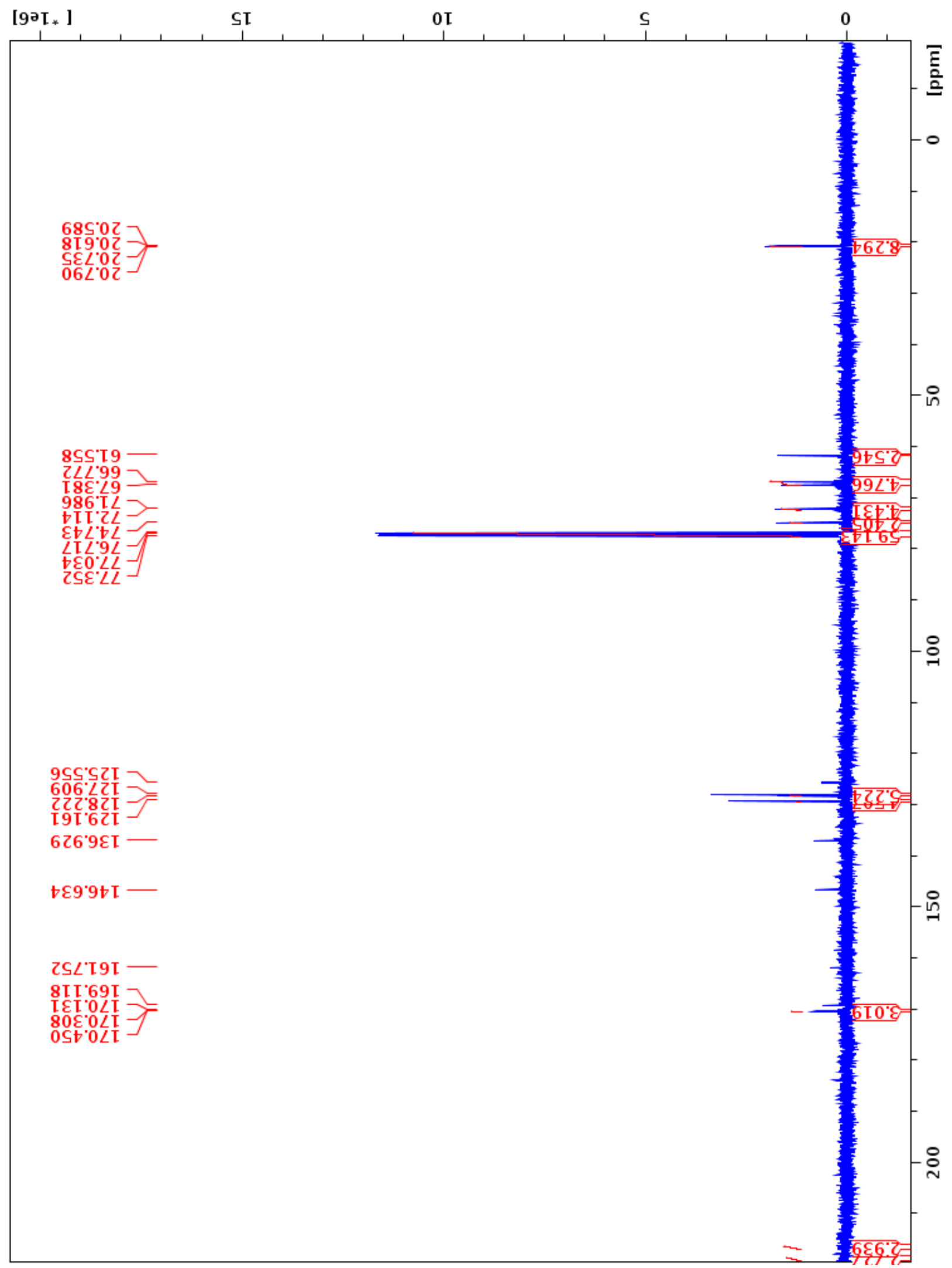


10e: 2-(3-chlorophenyl)-5-(2,3,4,6-tetra- $O$-acetyl-1-deoxy- $\beta$-D-galactopyranosyl)oxazole

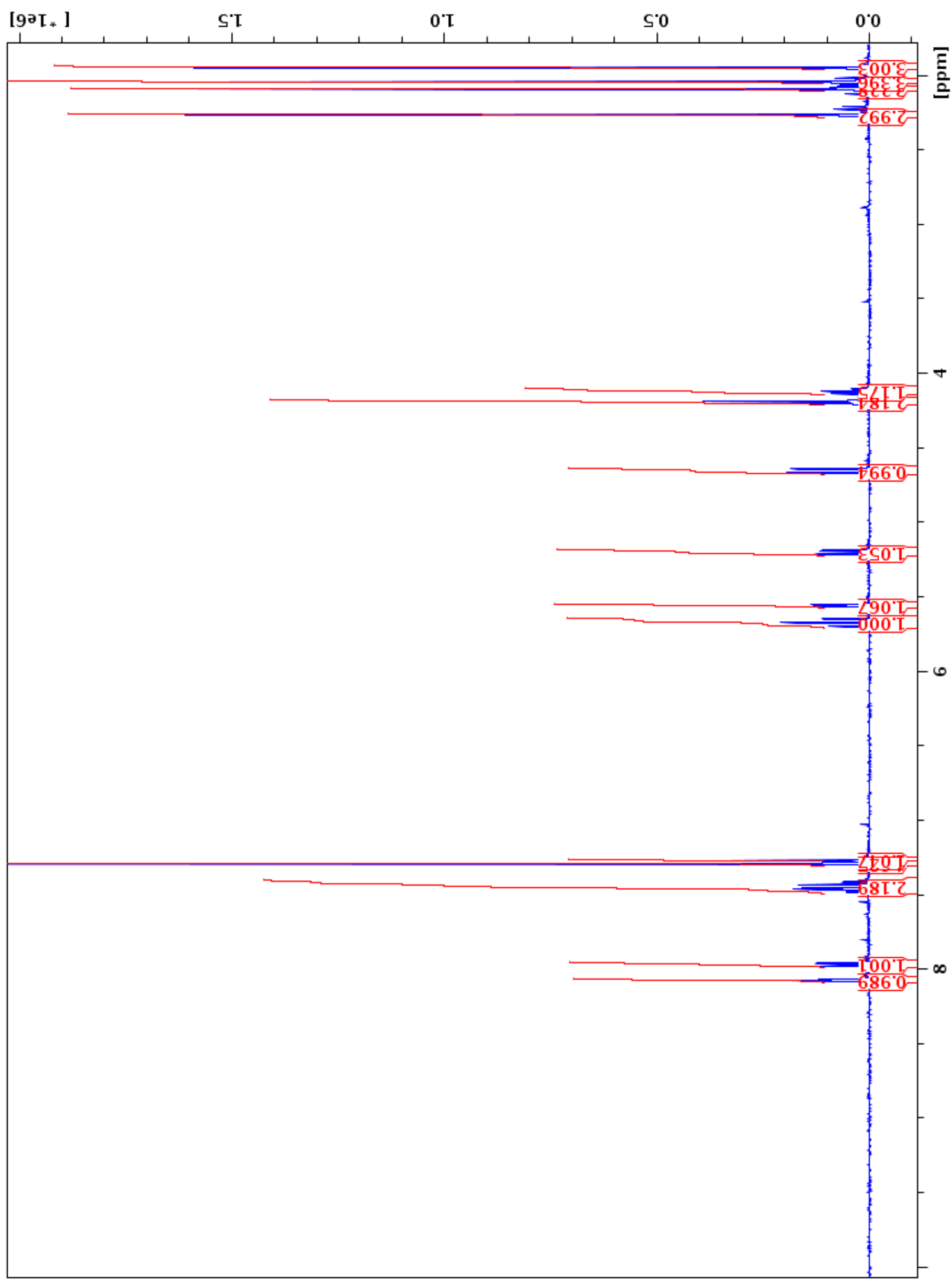


10e: 2-(3-chlorophenyl)-5-(2,3,4,6-tetra-O-acetyl-1-deoxy- $\beta$-D-galactopyranosyl)oxazole

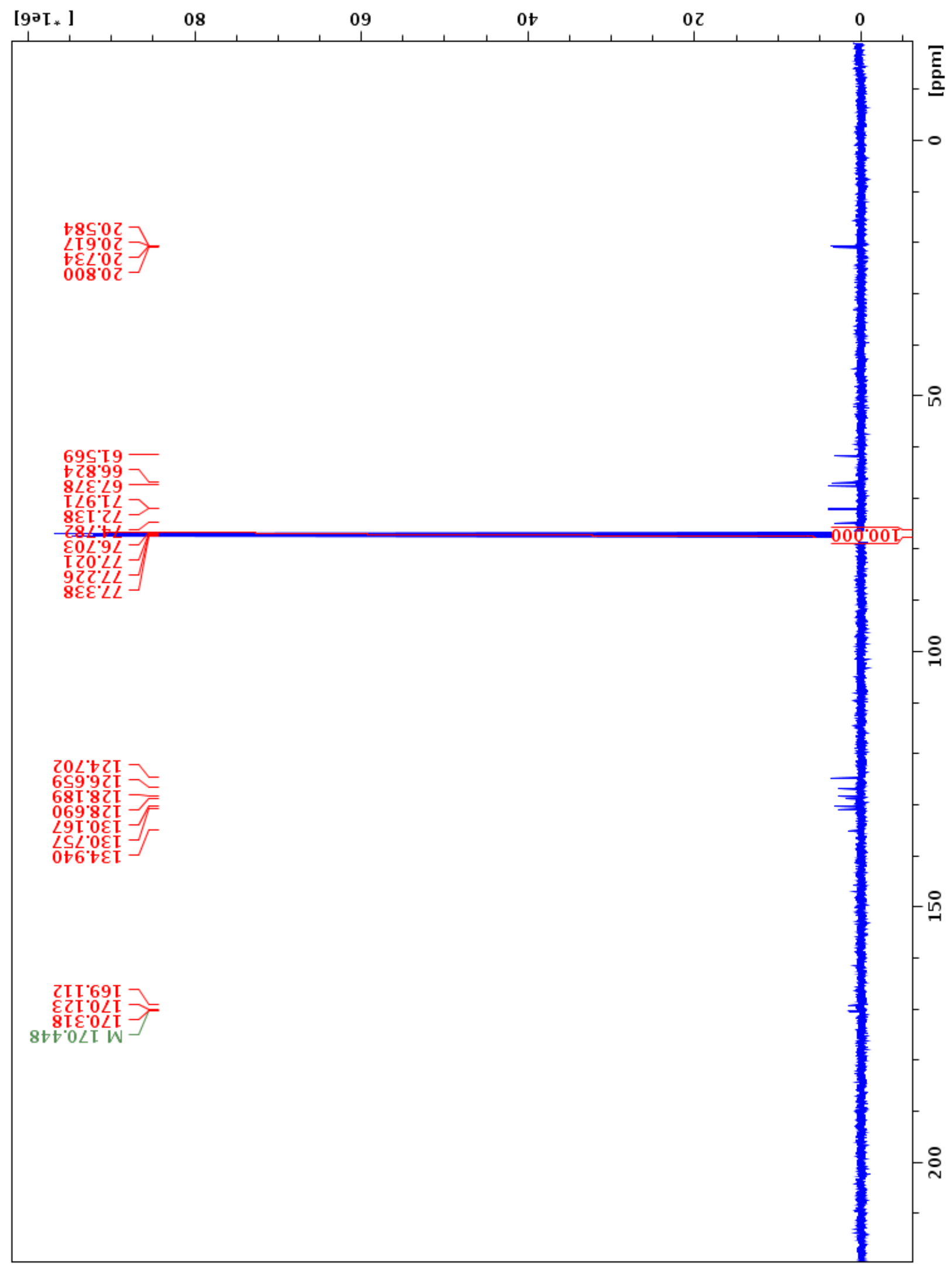


10f: 2-(4-methylphenyl)-5-(2,3,4,6-tetra- $O$-acetyl-1-deoxy- $\beta$-D-galactopyranosyl)oxazole

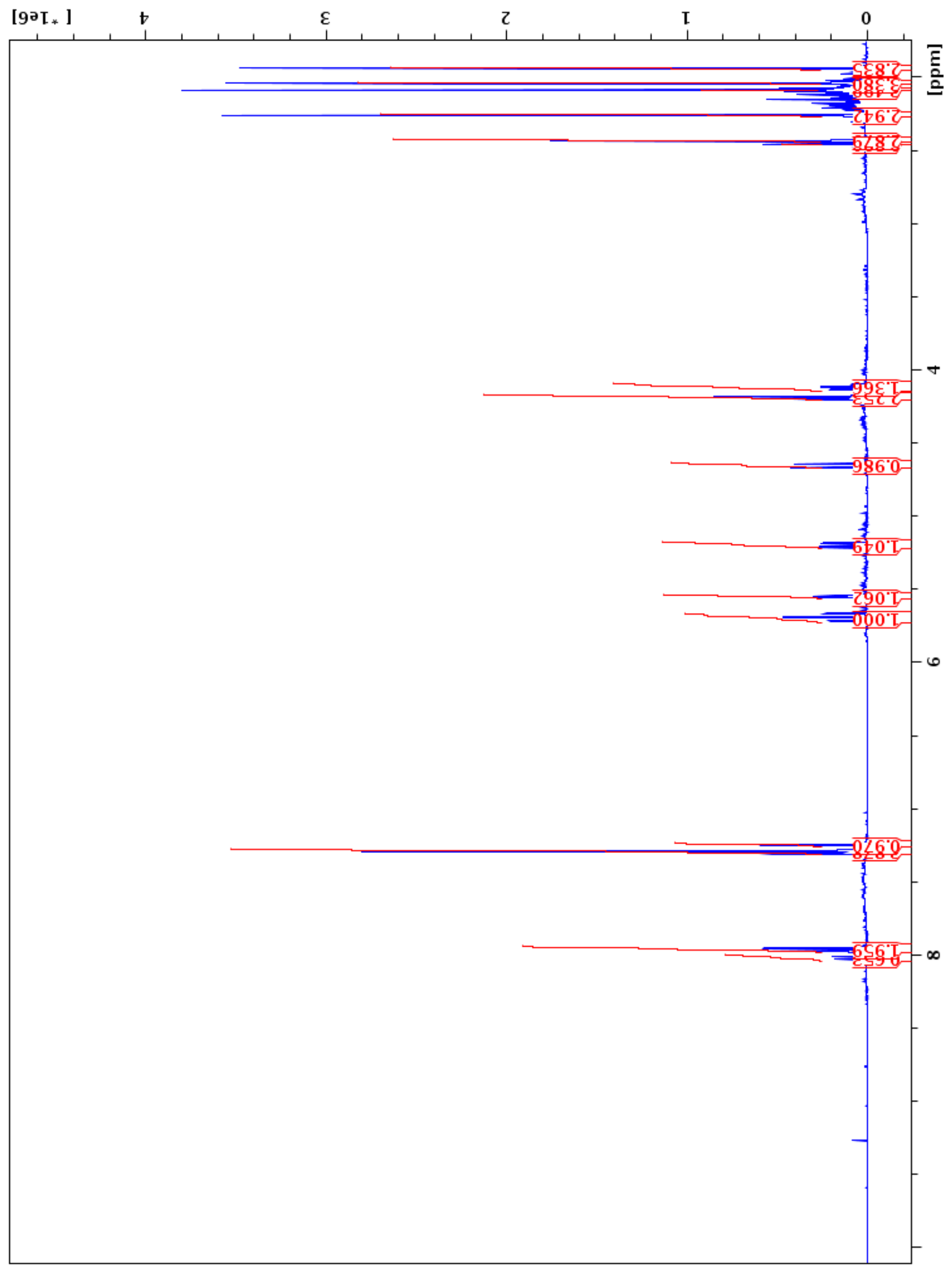


10f: 2-(4-methylphenyl)-5-(2,3,4,6-tetra- $O$-acetyl-1-deoxy- $\beta$-D-galactopyranosyl)oxazole

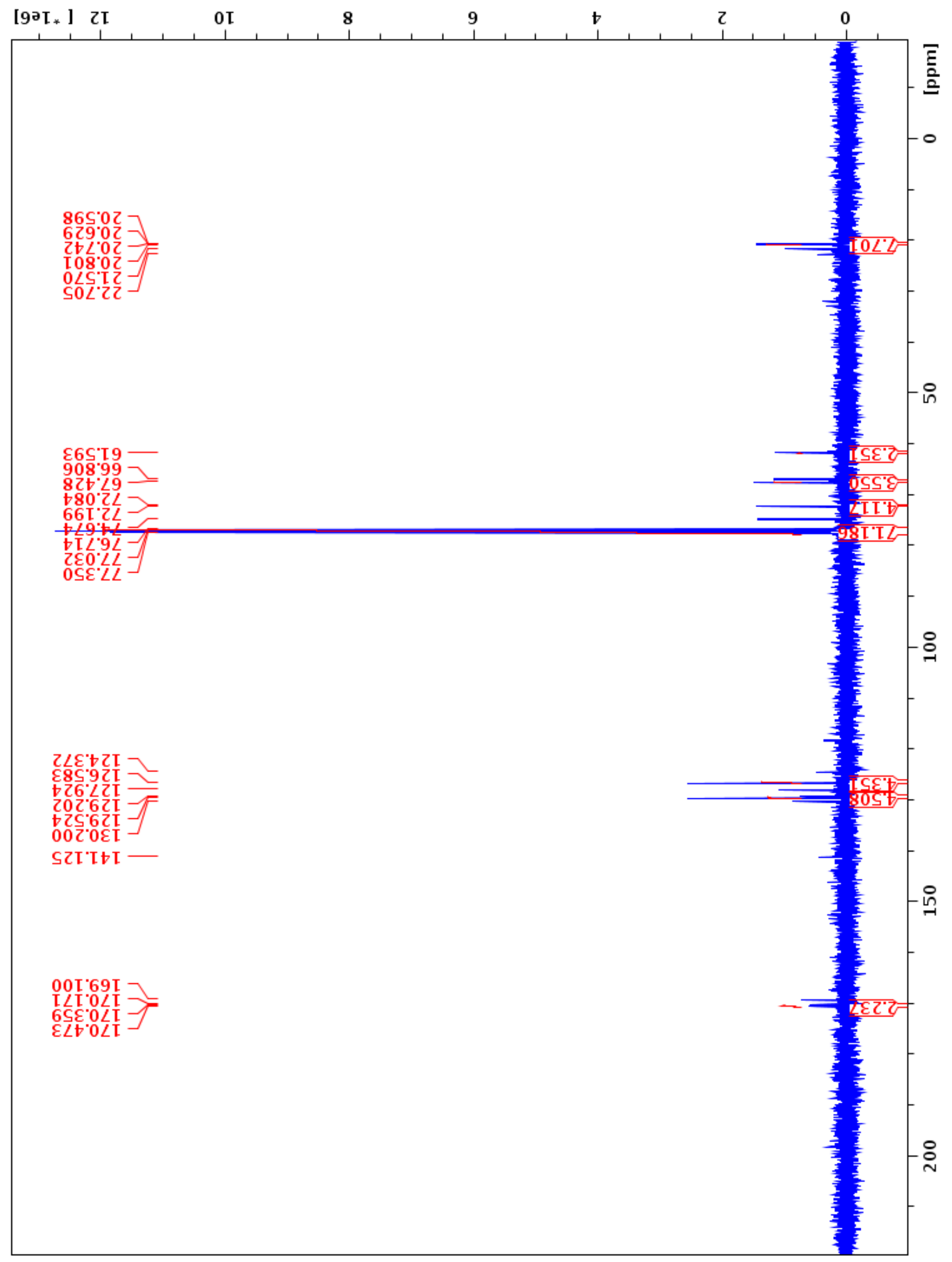


10g: 2-(3-methylphenyl)-5-(2,3,4,6-tetra-O-acetyl-1-deoxy- $\beta$-D-galactopyranosyl)oxazole

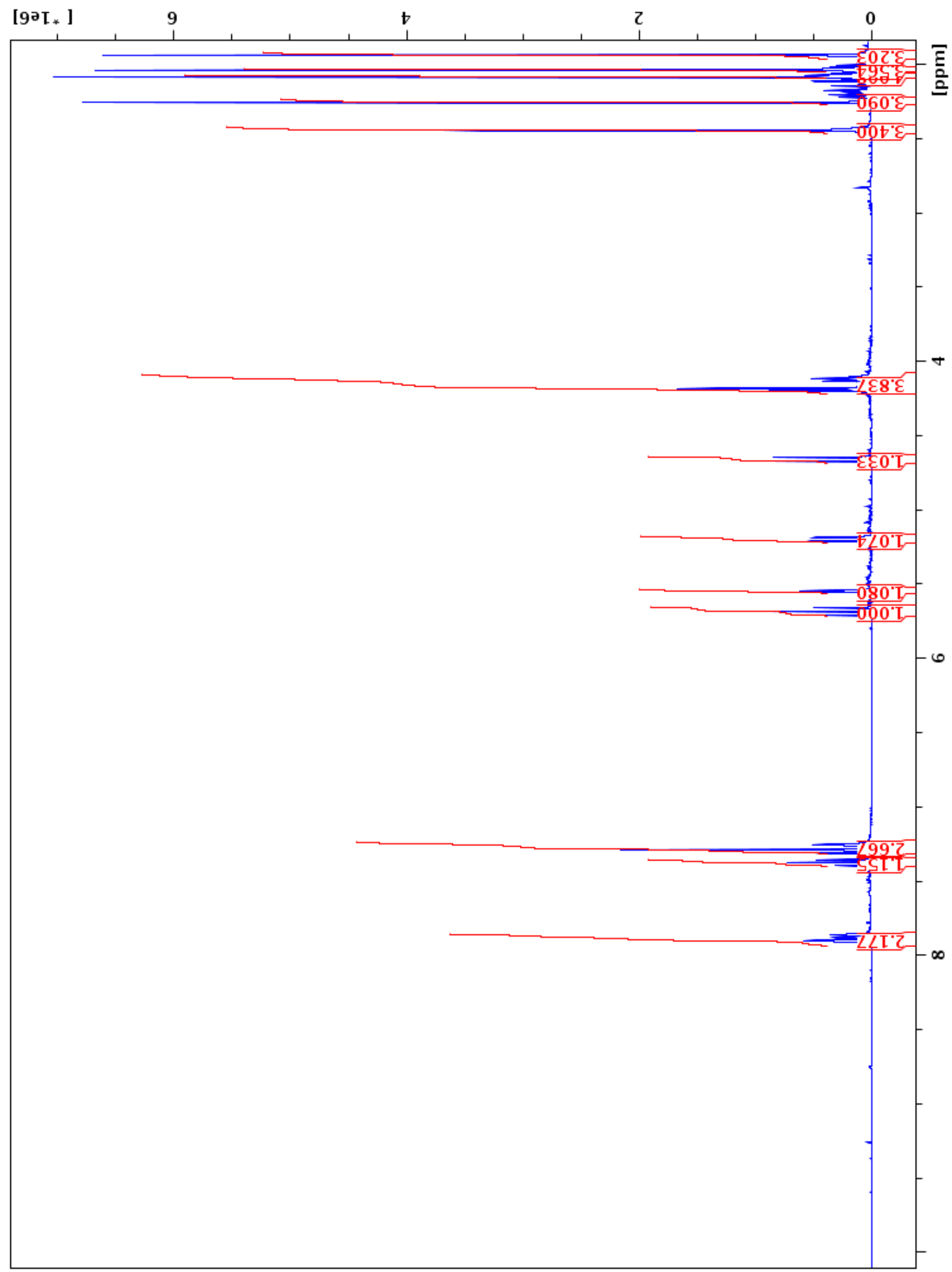


10g: 2-(3-methylphenyl)-5-(2,3,4,6-tetra-O-acetyl-1-deoxy- $\beta$-D-galactopyranosyl)oxazole

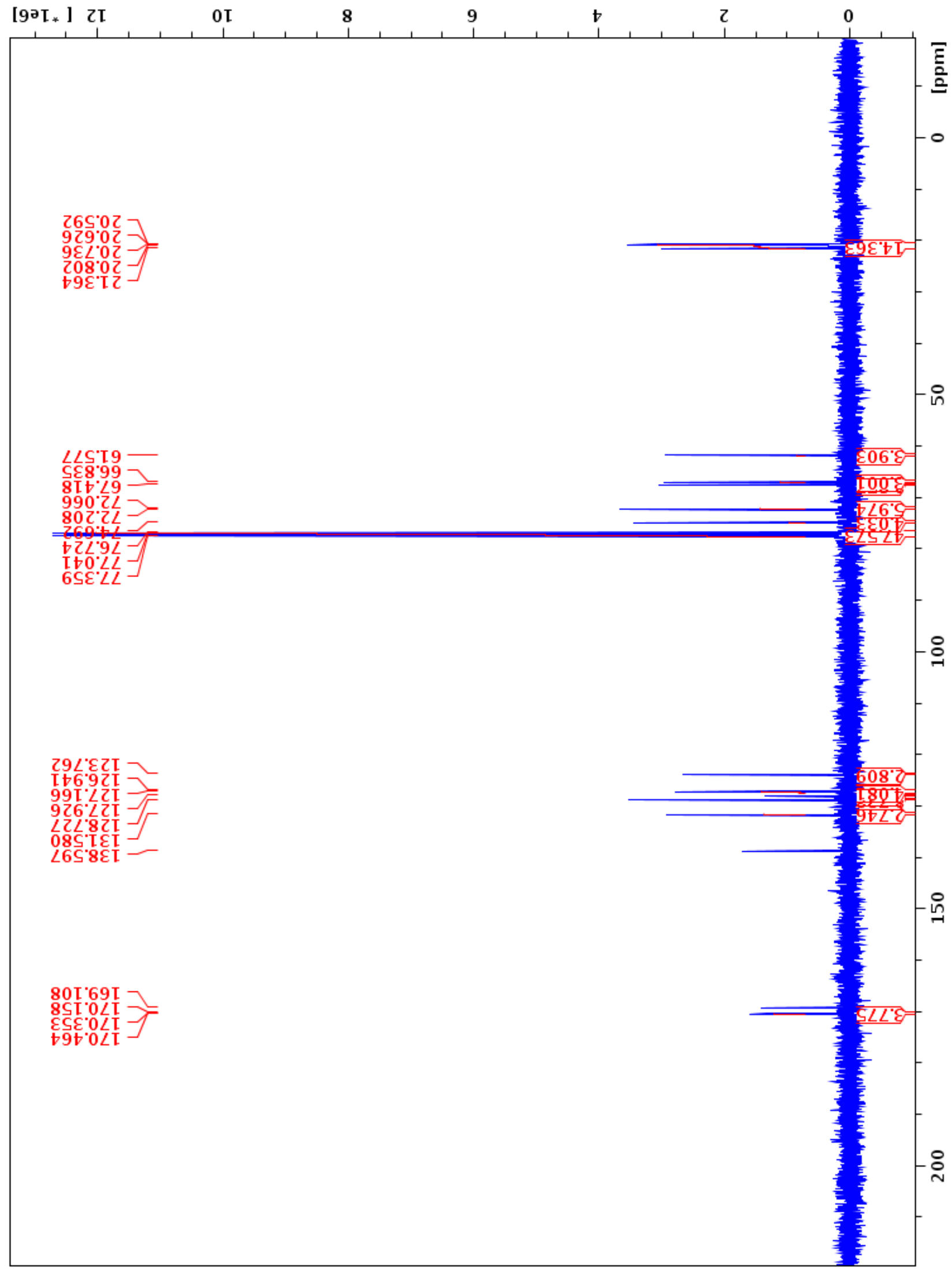


10h: 2-(4-fluorophenyl)-5-(2,3,4,6-tetra- $O$-acetyl-1-deoxy- $\beta$-D-galactopyranosyl)oxazole

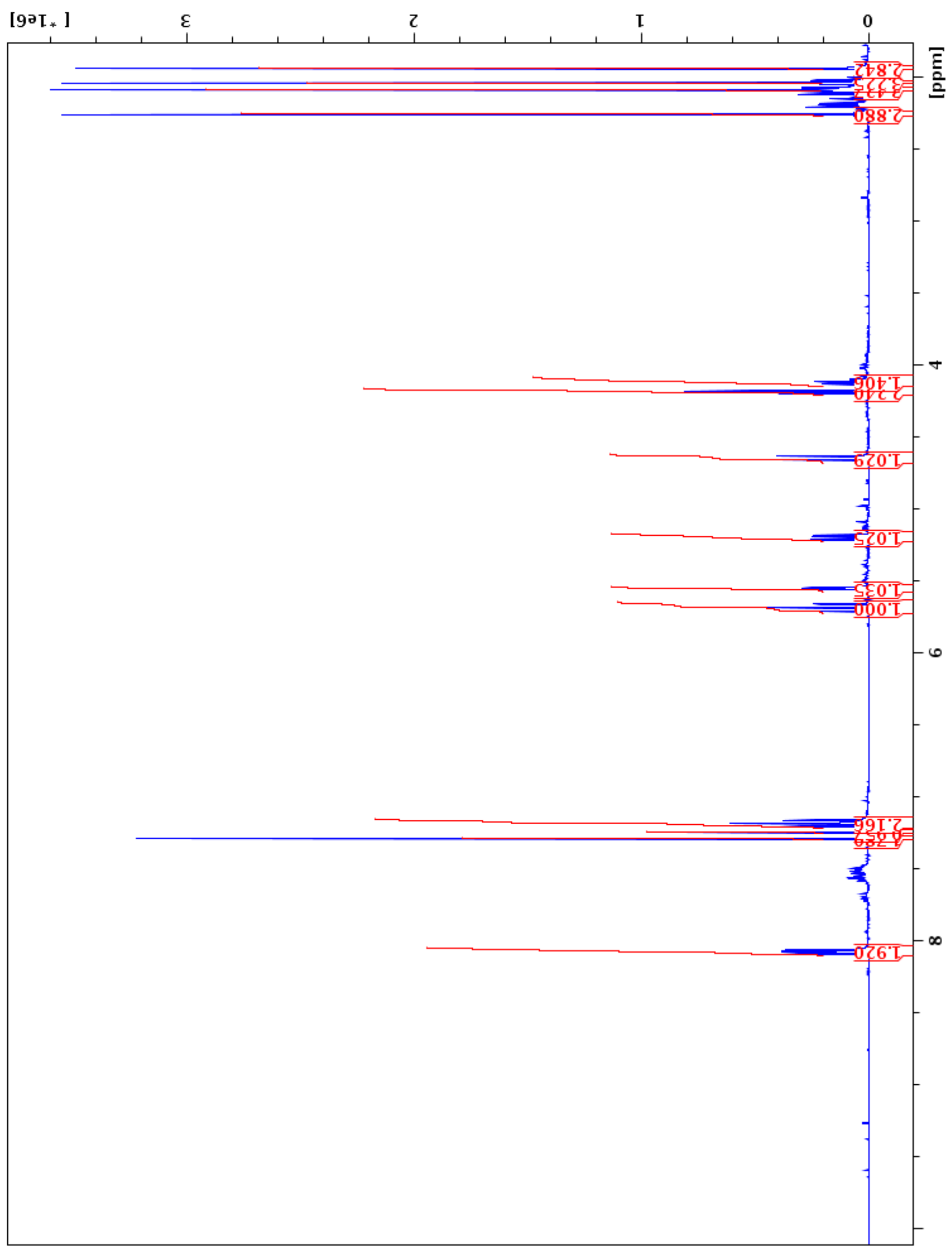


10h: 2-(4-fluorophenyl)-5-(2,3,4,6-tetra- $O$-acetyl-1-deoxy- $\beta$-D-galactopyranosyl)oxazole

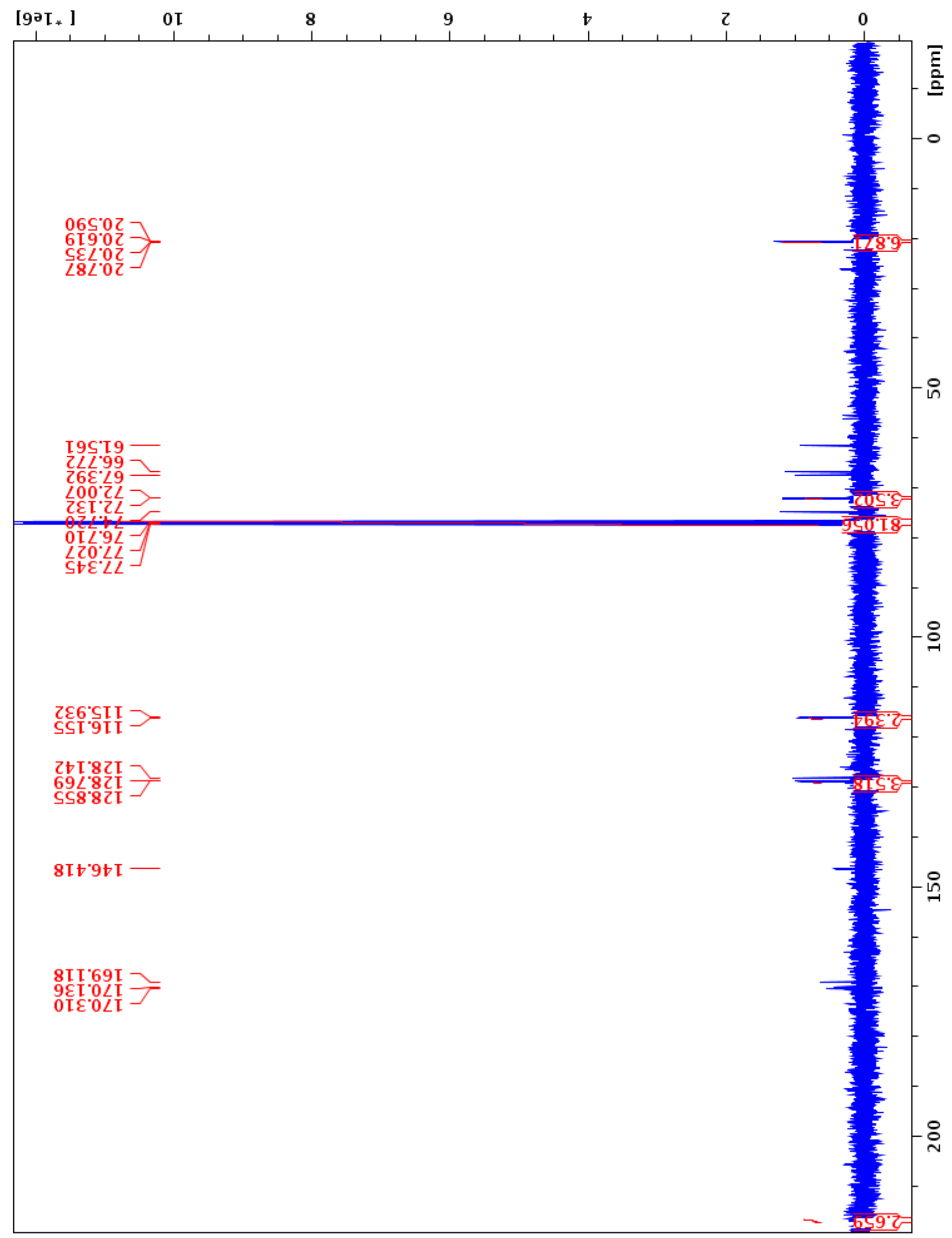


10i: 2-(3-fluorophenyl)-5-(2,3,4,6-tetra- $O$-acetyl-1-deoxy- $\beta$-D galactopyranosyl)oxazole

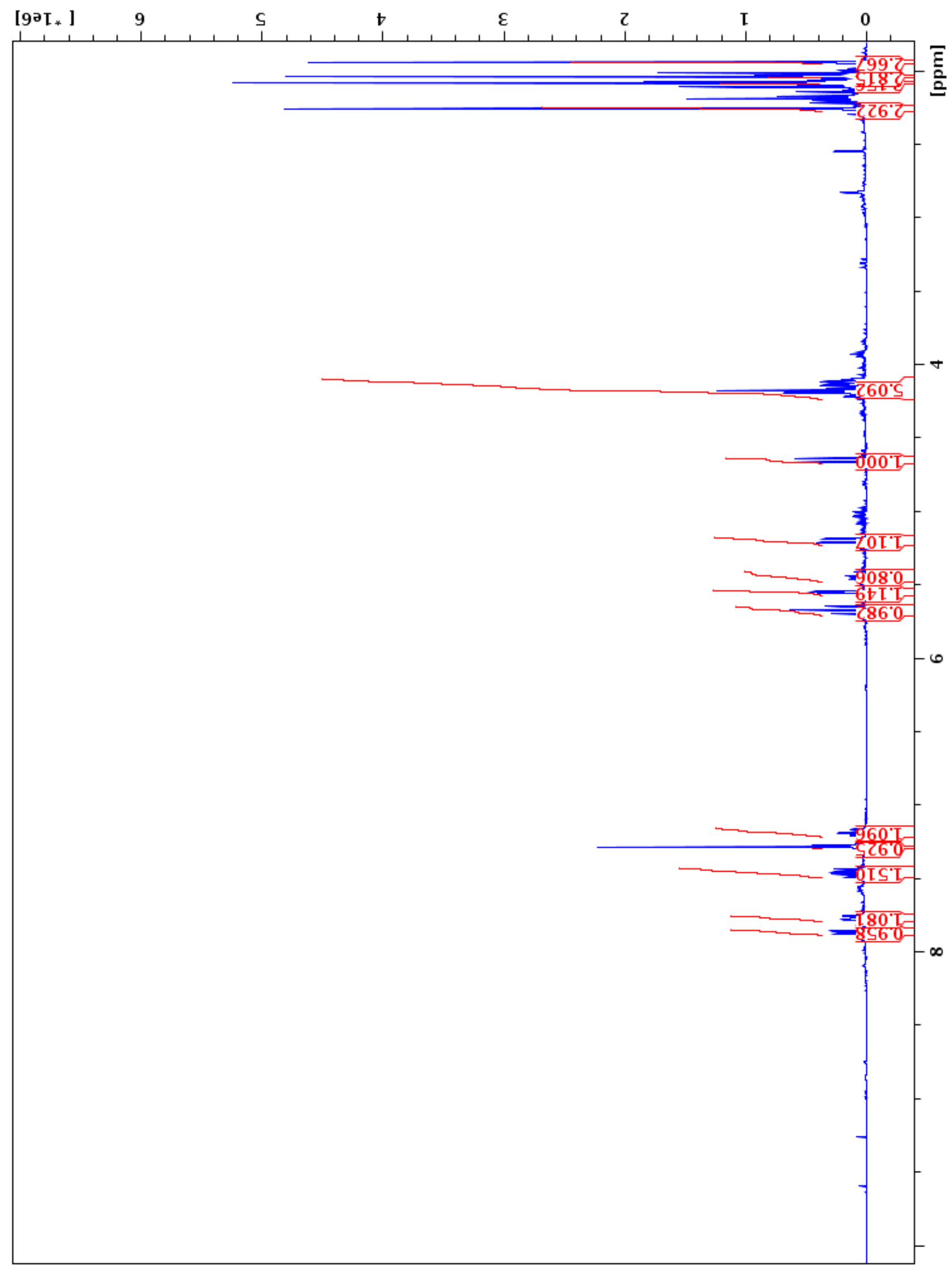


10i: 2 -(3-fluorophenyl)-5-(2,3,4,6-tetra- $O$-acetyl-1-deoxy- $\beta$-D-galactopyranosyl)oxazole

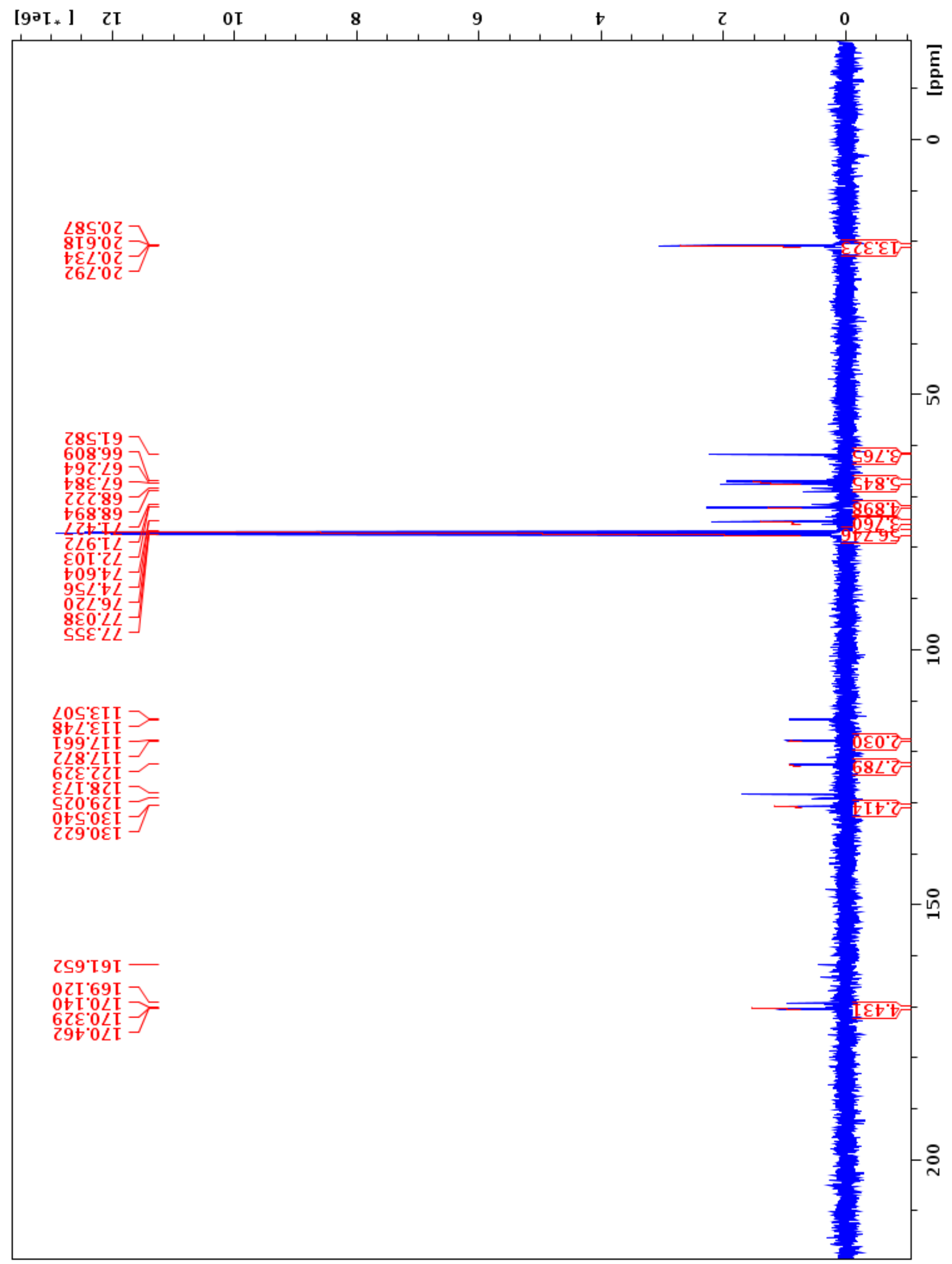

